\title{
ACTIVE LEARNING USING MODEL-ELICITING ACTIVITIES AND INQUIRY-BASED LEARNING ACTIVITIES IN DYNAMICS
}

\author{
A Thesis \\ presented to \\ the Faculty of California Polytechnic State University, \\ San Luis Obispo
}

\author{
In Partial Fulfillment \\ of the Requirements for the Degree \\ Master of Science in Mechanical Engineering
}

by

Jeffrey Phillip Georgette

December 2013 
(C) 2013

Jeffrey Phillip Georgette

ALL RIGHTS RESERVED 
COMMITTEE MEMBERSHIP

TITLE:

Active Learning using Model-Eliciting Activities and Inquiry-Based Learning Activities in Dynamics.

AUTHOR:

Jeffrey Phillip Georgette

DATE SUBMITTED:

December 2013

COMMITTEE CHAIR:

Brian P. Self, Ph.D

Professor, Mechanical Engineering

COMMITTEE MEMBER:

James Widman, Ph.D

Professor, Mechanical Engineering

COMMITTEE MEMBER: Andrew Kean, Ph.D

Associate Professor, Mechanical Engineering 


\section{ABSTRACT}

Active Learning using Model-Eliciting Activities and

Inquiry-Based Learning Activities in Dynamics.

Jeffrey Philip Georgette

This thesis focuses on a year-long project of implementing active learning in undergraduate dynamics courses at Cal Poly San Luis Obispo from 2012-2013. The purpose is to increase conceptual understanding of critical dynamics concepts and to repair misconceptions of the students. Conceptual understanding in Dynamics is vital to understanding the big picture, building upon previous knowledge, and better understanding the behavior of engineering systems. Through various hands-on activities, students make predictions, test their conceptions, and solve real world problems. These active learning methods allow students to improve their learning of Dynamics concepts. Education research on active learning is present in Physics and Mathematics disciplines, yet is still growing in Engineering.

Four Inquiry-Based Learning Activities (IBLAs) and two Model-Eliciting Activities (MEAs) are discussed in this thesis. Inquiry-Based Learning Activities feature student prediction and experimentation in which the physical world acts as the authority. On the other hand, ModelEliciting-Activities prompt students to solve real world problems and deliver results to a client.

From the results, some activities yield an increase in conceptual understanding, as measured by assessment items, while others do not yield a significant increase. These activities not only help to promote conceptual gains, but also to motivate students and offer realistic engineering contexts. In conclusion, the six total IBLA and MEAS will continue in practice and be improved in their implementation.

This thesis work will contribute to engineering education research of active learning methods, and improve the undergraduate dynamics curriculum locally at Cal Poly.

Keywords: dynamics, active learning, engineering education, inquiry-based learning, Model-eliciting activity, education research 


\section{ACKNOWLEDGMENTS}

I greatly thank Dr. Brian Self for his support, guidance, feedback, suggestions for professional improvement, and encouragement throughout the year. I would also like to thank Dr. James Widmann for explaining concepts, guidance, and his enthusiasm. Thanks to Dr. Andrew Kean for his guidance and support. Thanks to Dr. Michael Prince for offering feedback and support for the research.

Thanks to Kathryn Bohn, Alex Baucom, Michael Hoover, Baheej Saoud, and Morgan Zandonella for their work on the project and enthusiasm. I thank my parents for their support in multiple forms.

Support for this work was funded by the National Science Foundation, NSF 1044282, Using Inquiry-Based Activities to Repair Student Misconceptions in Engineering Dynamics and NSF 717595, Collaborative Research: Improving Engineering Students' Learning Strategies Through Models and Modeling. Any opinions, findings, and conclusions or recommendations expressed in this material are those of the author(s) and do not necessarily reflect the views of the National Science Foundation. 


\section{TABLE OF CONTENTS}

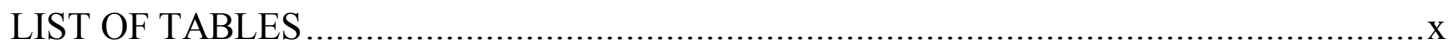

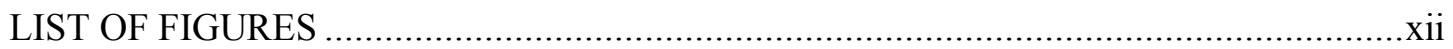

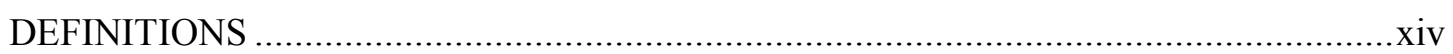

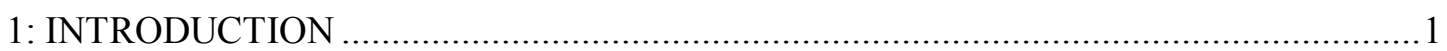

Conceptual Understanding in Dynamics Classes ...................................................... 1

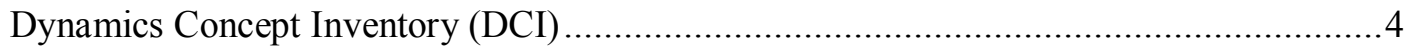

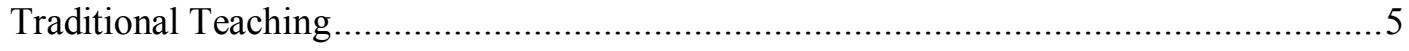

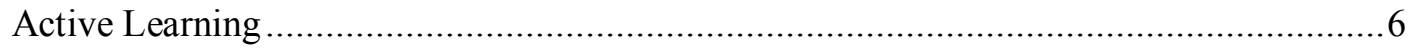

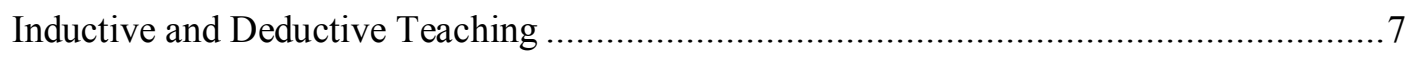

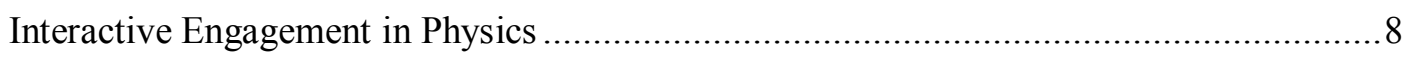

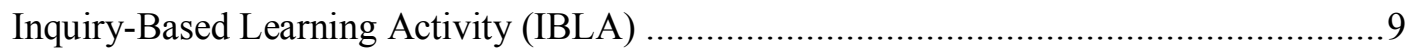

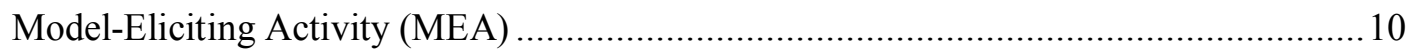

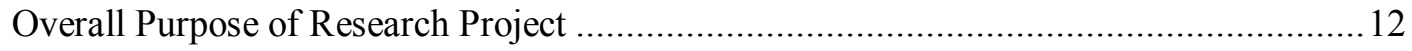

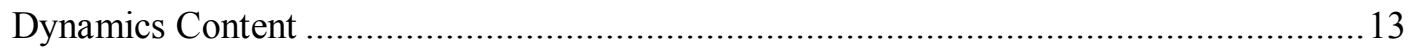

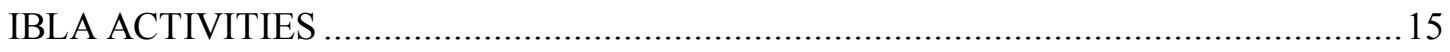

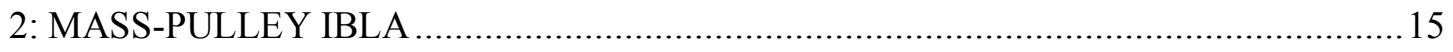

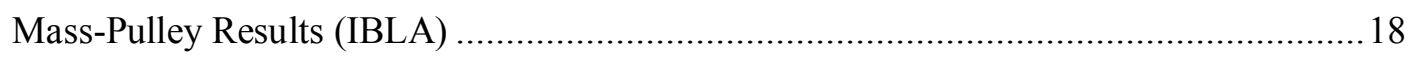

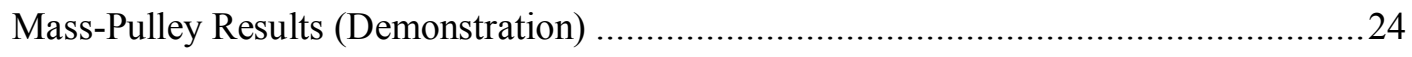

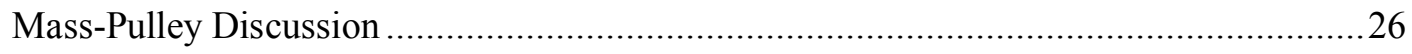




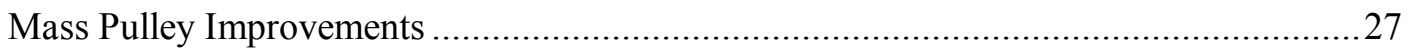

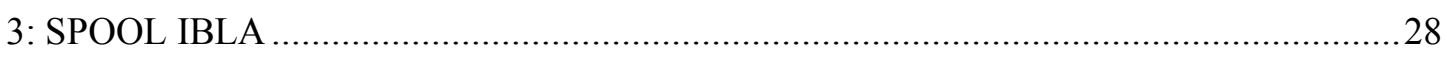

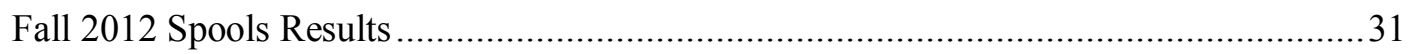

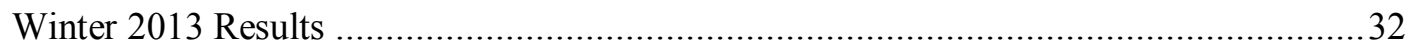

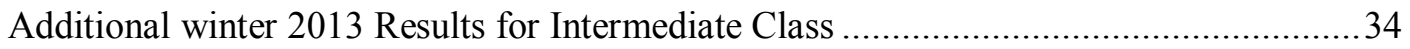

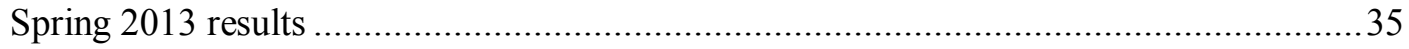

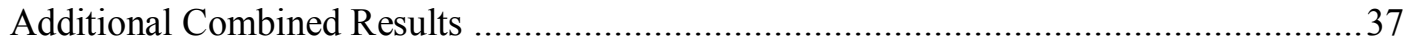

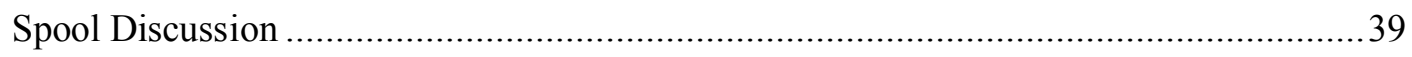

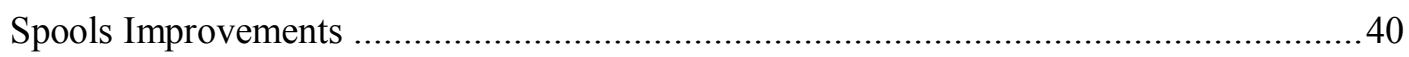

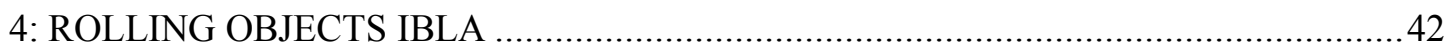

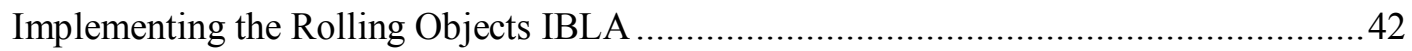

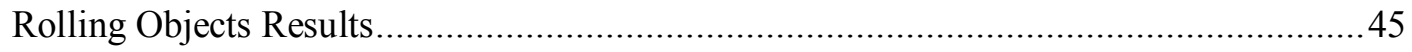

Implementing the Rolling Objects Demonstration ................................................. 46

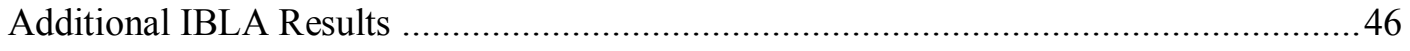

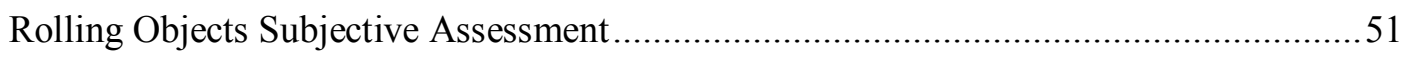

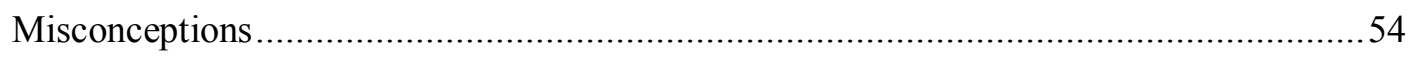

Compare Demonstration to Small Group IBLA ...................................................... 54

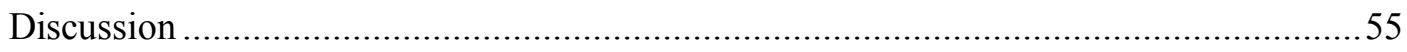

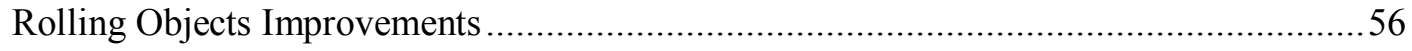

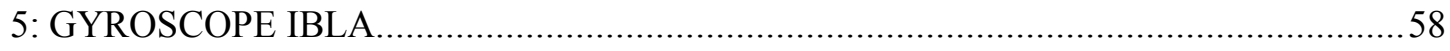

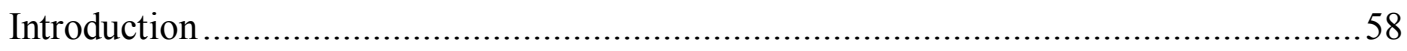




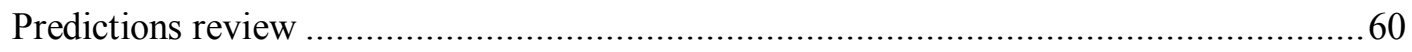

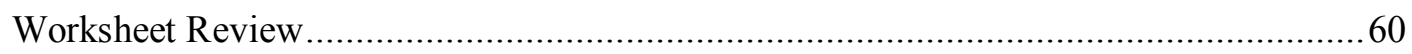

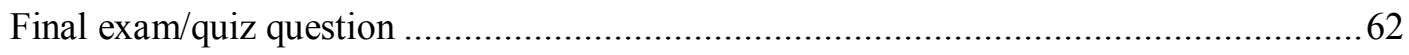

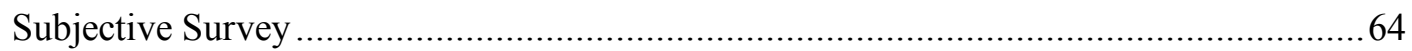

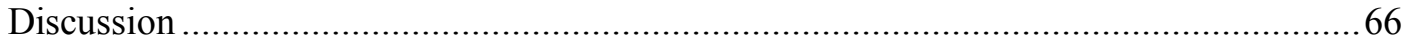

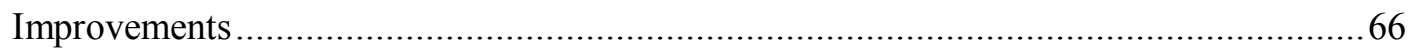

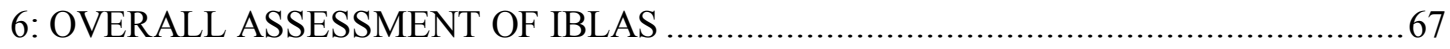

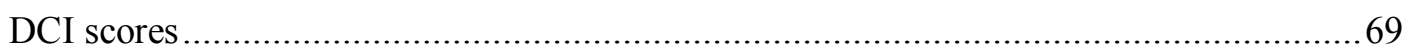

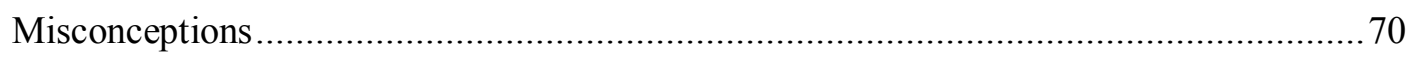

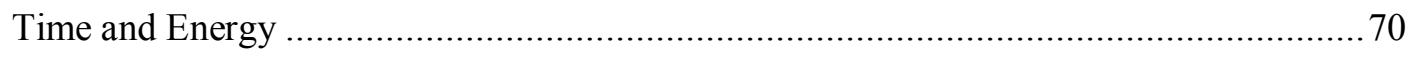

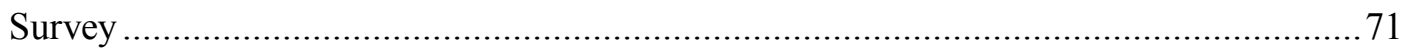

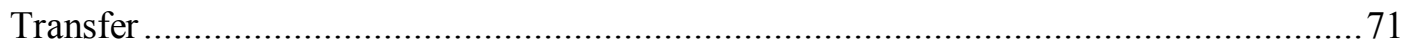

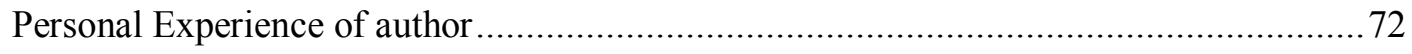

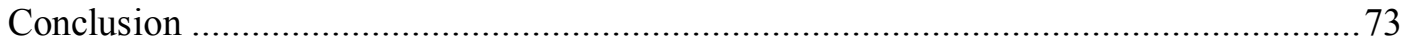

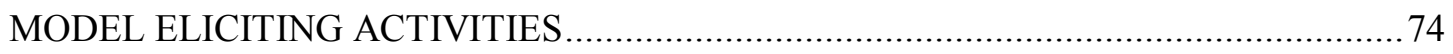

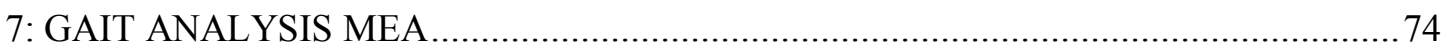

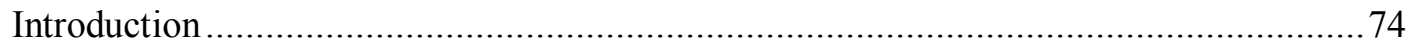

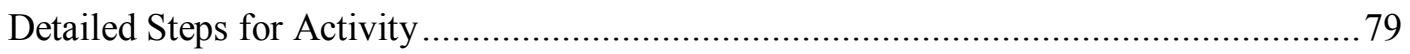

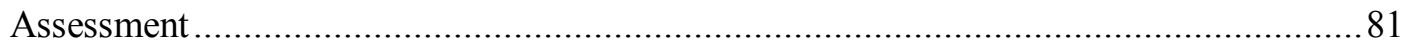

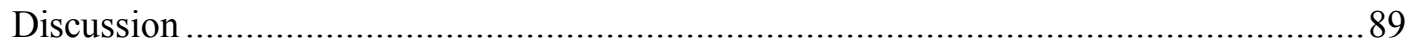

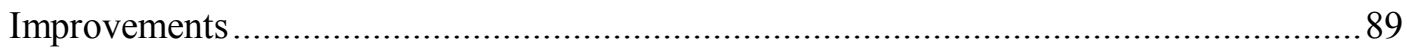




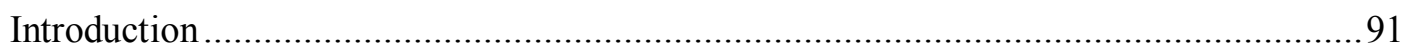

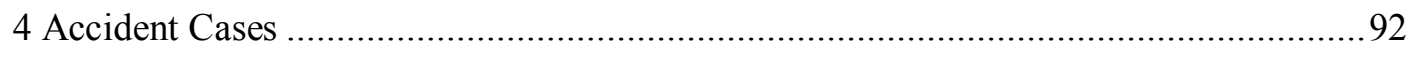

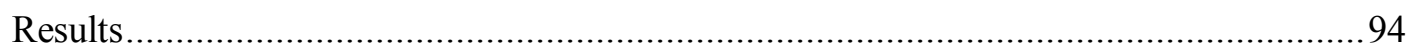

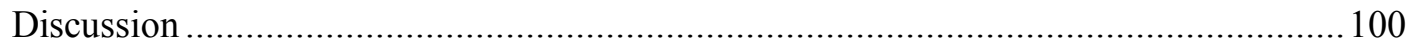

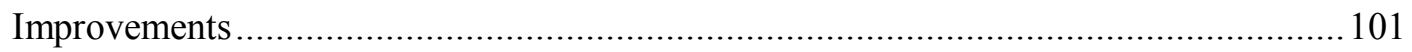

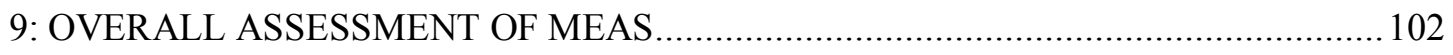

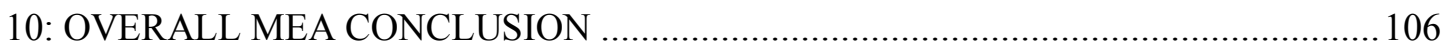

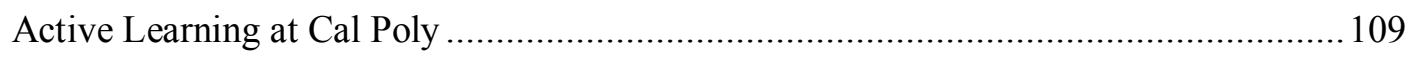

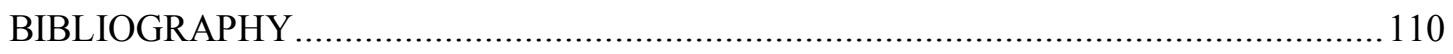

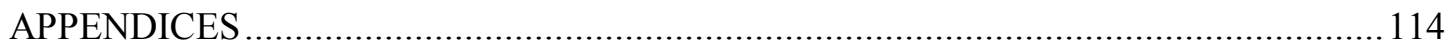

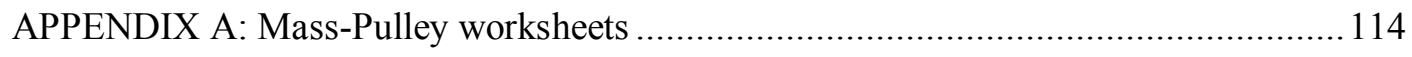

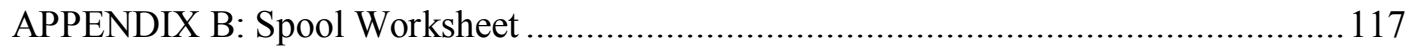

APPENDIX C: Rolling Objects Worksheets ..................................................... 119

APPENDIX D: DCI Question for Rolling Objects.............................................. 122

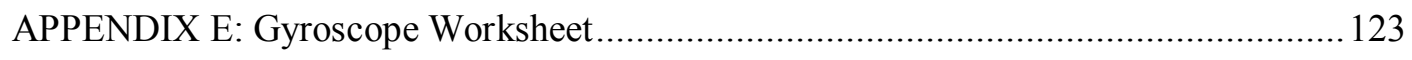

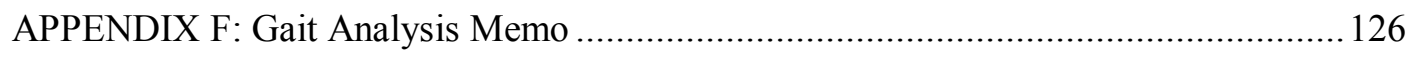

APPENDIX G: Matlab Code for Gait Analysis MEA .................................................. 128

APPENDIX H: Vehicle Accident Reconstruction MEA memo ..................................... 140

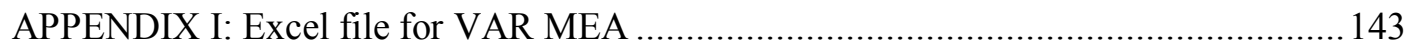




\section{LIST OF TABLES}

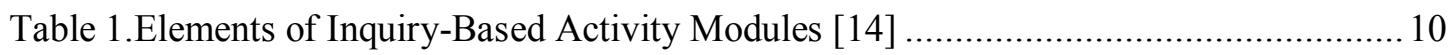

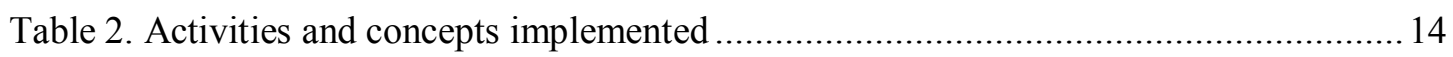

Table 3. DCI dealing with Mass-Pulley Concept, for multiple Introductory Dynamics

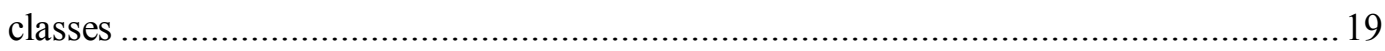

Table 4. Mass-Pulley Results from team inquiry, Engineering Dynamics class ...................20

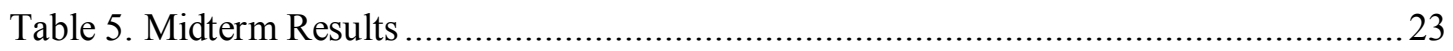

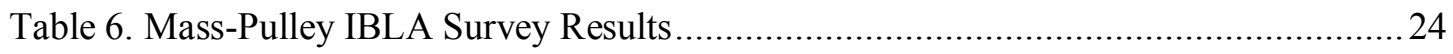

Table 7. Mass-Pulley demonstration results for Intermediate Class ...................................25

Table 8. Assessment of Spool IBLA in fall '12; Percentage of students answering the

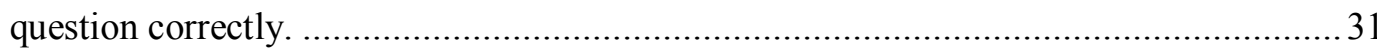

Table 9. Assessment of Spool IBLA Winter 2013; Percentage of students answering the

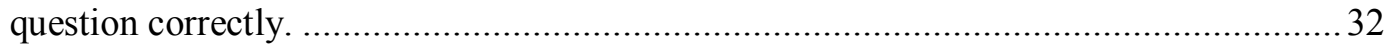

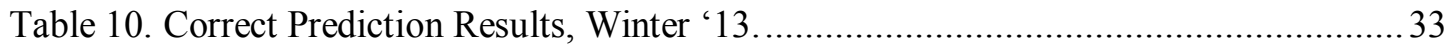

Table 11. Results from Intermediate dynamics, Winter '13; correct responses ......................34

Table 12. Percent Correct Scores from Spools in Intermediate dynamics, Winter' 13 ............. 35

Table 13. Percent Correct scores from Spools in Spring '13, Engineering Dynamics class.... 36

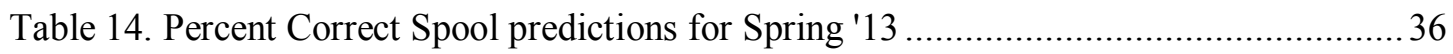

Table 15. When did the spool concepts make sense to you? Engineering Dynamics class .... 38

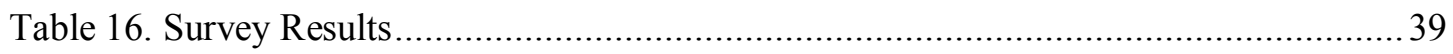

Table 17. (a) The pre- and post-DCI results of the rolling objects question along with normalized gain, (b) the quiz results from the day before the IBLA, and (c) the results

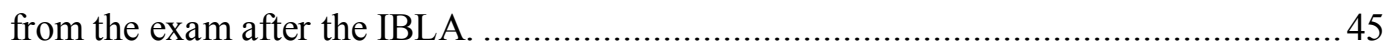

Table 18. Prediction Questions shown as pictures........................................................ 47 
Table 19. Predictions for object races, by class and question \# (see Table 18). (intm) $=$

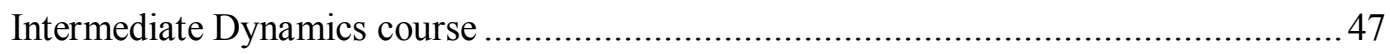

Table 20. Categorizing student in-class worksheet responses Fall'12 IBLA .........................50

Table 21. Midterm question assessment, Spr '13 Intermediate class .................................. 51

Table 22. Student responses as to when they understood the concepts in the IBLA.

(Engineering Dynamics only, data collected from survey) ........................................52

Table 23. Results from end of the course survey (no intermediate class survey data)............53

Table 24. Prediction correct percentages, winter and spring 2013 ...................................6 60

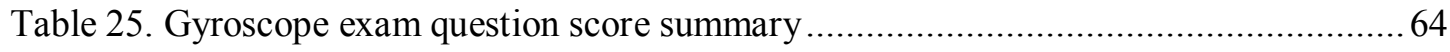

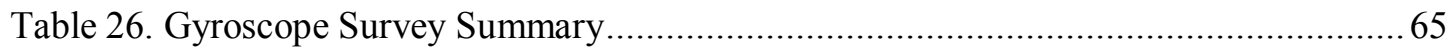

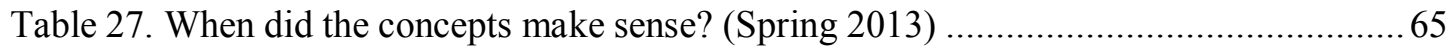

Table 28. DCI scores from dynamics classes, including both IBLA and non-IBLA

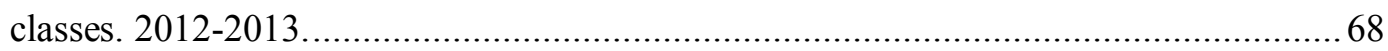

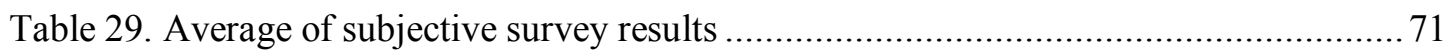

Table 30. Summary of ranking methods. The left column displays the type of ranking used, the second and third columns count the number of groups that represented such

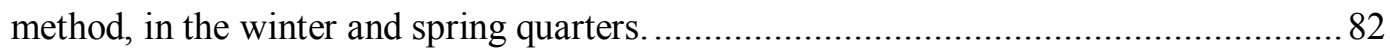

Table 31. Gait Activity Survey results from winter quarter, last entry is from spring

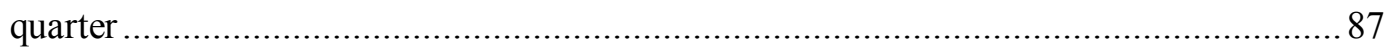

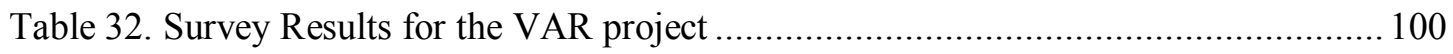




\section{LIST OF FIGURES}

Figure 1. Active-engagement vs. traditional instruction for improving students' conceptual understanding of basic physics concepts (taken from Laws et al.,[14]). Traditional instruction led to a gain of student's conceptual knowledge by 5 to $15 \%$, while active learning results in up to $90 \%$ of students understanding concepts. ............... 7

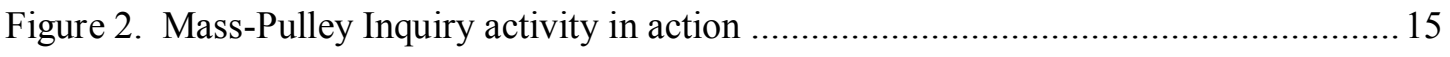

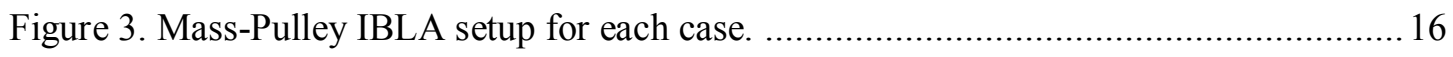

Figure 4. DCI Question \#13: Which 10N block will have a larger acceleration? .................. 17

Figure 5. Midterm Problem where students transfer the concepts of Newtons $2^{\text {nd }}$ Law to the case of a seesaw, with a weight force on case 'A' and a mass-less force on case 'B'.

Figure 6. Plot of weight ratios to acceleration of block 22

Figure 7. Spool diagram for both cases: horizontal pull (left) and vertical pull (right) [20] .28

Figure 8 . Spool activity is rolling into action! 29

Figure 9. Automobile tire friction question on DCI: Find the friction force direction and expression for the front and rear tires [9]. 29

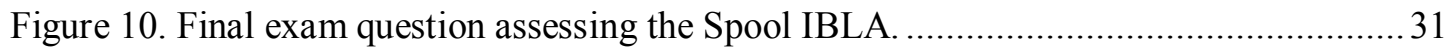

Figure 11. Diagrams from Quiz in winter 13. Part a) on Left. Part b) on right ................ 32

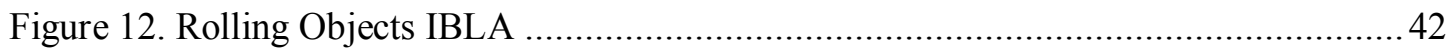

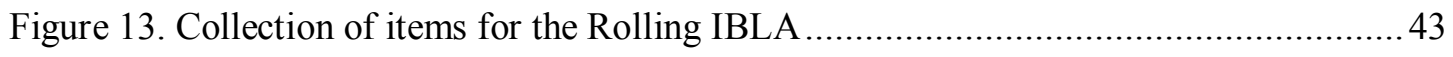

Figure 14. Midterm ranking question, Spring '13 intermediate. .......................................51

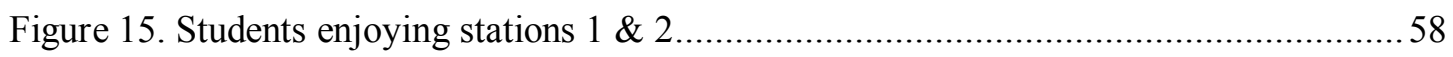

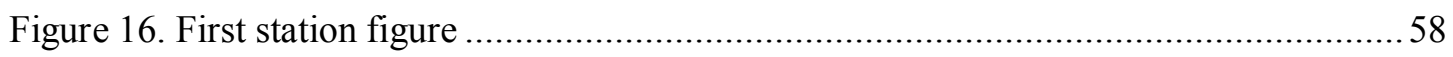

Figure 17. Students working through station two in the Gyroscope IBLA.........................59 
Figure 18. Precession occurs as person spins, they apply a counter moment to the wheel to keep it in place. Station 3a.

Figure 19. Final exam question one

Figure 20. Final exam question two, Rotating turbine in jet. From Meriam and Kraige, 2006

Figure 21. Leg model with limbs 74

Figure 22. Video markers during motion 75

Figure 23. Position vectors, Free Body Diagram, and Kinetic Diagram of lower leg 77

Figure 24. Data analysis progression: Record Video footage and collect force plate data, then digitize joint positions into a Model, then use Matlab to solve for loads and plot results.

Figure 25. Flowchart of measuring motion data and solving for moments and forces in the knee

Figure 26. Winter Velocity Results. Each colored dot represents a velocity data point from one team, two dots stacked represents a range.

Figure 27. Spring velocity results. Each colored dot represents a velocity data point from one team, two dots stacked represents a range.

Figure 28. Exam Score Comparison between MEA and traditional classes.

Figure 29. Vehicle Accident sign convention. 144 


\section{DEFINITIONS}

Kinematics - dynamics problems in which motions, positions, and velocity, are analyzed

Kinetics - dynamics problems in which forces and moments are analyzed in addition the kinematics

FBD: Free Body Diagram, which includes forces and moments applied to the body

MAD: Mass Acceleration Diagram, which includes mass*acceleration of the center of gravity and also the moment of inertia*angular accelerations.

KD: kinetic diagram, same as MAD.

DCI: Dynamics Concept Inventory

IBLA: Inquiry-Based Learning Activity

MEA: Model-Eliciting Activity

VAR: vehicle accident reconstruction

Moment of inertia (MOI): the mass quantity of a body which is resistant to angular acceleration

Center of gravity (CG): the point on a rigid body where the total mass may be thought to be concentrated. Usually the KD references this point. 


\section{1: INTRODUCTION}

Students spend considerable effort to learn course concepts in hope of becoming competent engineers. They put effort into learning lecture material, collaborating on projects, and working through example problems. Along the way learners develop their problem solving ability, teamwork skills, and hopefully develop a strong conceptual understanding of engineering principles. One of these outcomes stands out to the Cal Poly dynamics research team-conceptual understanding, which is critical in becoming both a competent engineering student and a practicing professional [32]. Students taking engineering courses must understand the concepts, fulfill course requirements, and work cooperatively to succeed [29]. In order to yield meaningful learning in the classroom and labs, students must be engaged in the class experience.

Students can engage in the engineering course material through hands-on activities in which they experiment with physical objects: by rolling metal cylinders, dropping metal weights, unwinding plastic spools, and spinning gyroscopes. Such hands-on experiments create a personal, relatable and clear learning experience for the students. From participating in the hands-on experiment, students can agree with the behavior of these physical systems or be surprised by the counterintuitive results, both of which promote learning gains. Along the way, students constantly reorganize and refine their conceptual understanding. Understanding conceptual knowledge is "critical to the development of competence in engineering students and in practicing professionals" [32].

\section{Conceptual Understanding in Dynamics Classes}

Conceptual knowledge, according to Rittle-Johnson, is stated as "understanding of principles governing a domain and the interrelations between units of knowledge in a domain" [24]. A domain can be, for example, the rolling motion of an object, where the behavior is governed by Newton's second law, which relates forces and accelerations. One can prove correct conceptual 
understanding by accurately identifying what will happen in a real life physical situation. For example, accurately predicting the outcome of an experiment involving two moving objects that are connected together, recognizing applicable forces acting on an object, or predicting resultant motion of a rigid body from applied forces and moments, can indicate conceptual understanding. Engineers and students can show competency by analyzing these physical situations with engineering principles (such as work-energy, kinematics, kinetics, and impulse-momentum) and also by transferring their knowledge to future contexts.

Students assemble pieces of information and their own observations together into an organized structure. Minstrell describes identifiable pieces of students' knowledge as "facets." A facet is a convenient unit of thought, a piece of knowledge, or a strategy seemingly used by the student in addressing a particular situation [19]. Students also use 'constructs' of their knowledge. As a research team, (myself, two professors, and three undergrad students) we scrutinize students' facets and constructs when assessing their understanding and their logic; we also try to pinpoint the specific misconceptions. Part of the motivation for this research is to help students build a coherent framework of conceptual understanding. The National Research Council's study discusses that students need to organize new facts and knowledge within a unifying conceptual framework [4].

Conceptual knowledge is important for the following reasons. Bransford et al. states that "helping students acquire conceptual knowledge can also help them build more expert-like knowledge structures" and that "learning with conceptual understanding can enhance transfer of knowledge to new problems" [4]. Rittle-Johnson discusses how conceptual knowledge may enhance procedural knowledge and performance [25]. See Streveler et al. [32], section I, for a good description of why conceptual knowledge is important. 
a) Even though engineering students may grasp the basic course principles, they may still have misconceptions. Instructors must seek to repair their students' misconceptions and build correct understanding. Misconceptions

Students unfortunately bring misconceptions into the classroom, which the instructor seeks to rectify. As described in How People Learn, students build their view of the world from experiences and observations over prolonged periods of time. Even though these concepts may conflict with the physical world, students will maintain these concepts because it allows them to explain phenomena and make predictions about the physical world [4]. Misconceptions may originate from real experiences in life, and this can contribute to the difficulty in repairing them. For example, a basketball may roll faster down a hill than a tennis ball (although they have the same hollow shape); an apple may fall from a tree to the ground faster than a leaf (although they have the same acceleration in the absence of air resistance); two football players may collide and the smaller player may get hurt more than the larger player (although an equal force is exerted on both players). These examples and other misconceptions must be corrected for students to succeed in the future - if not corrected they may resurface months or years later. According to Bransford, "a key aspect of the new ways of teaching science is to focus on helping students overcome deeply rooted misconceptions that interfere with learning" [4]. This thesis work is dedicated to overcoming student-held misconceptions in undergraduate dynamics.

\section{b) Challenges}

In addition to misconceptions, there exist other challenges to teaching concepts. Students may have difficulty learning conceptual knowledge of certain engineering topics which are nonobservable and non-intuitive. Students often have trouble visualizing the friction force acting on an object or moment of inertia of an object, phenomena that are not directly observable. In addition, students cannot touch phenomena such as "energy" or feel "work" physically. Rather, 
they have an easier time seeing the velocity of a moving object or feeling tension in a rope, phenomena which are observable. Some concepts are not intuitive, and therefore challenging to teach, such as 3D gyroscopic motion. This research project seeks to teach non-intuitive, nonobservable, and challenging concepts through the use of active learning methods which will be explained later.

Educators have worked towards promoting conceptual understanding in the realm of college physics [14] and mathematics, although more work can be implemented in the engineering realm to realize learning gains. To assess conceptual understanding gains, the Foundation Coalition has developed such instruments as the Concept Inventory to identify students' misconceptions concerning important topics in the STEM fields [7]. The Force Concept Inventory has been used in physics classrooms to assess student conceptual understanding of force, velocity, and acceleration [24].Within the engineering community the force-concept-inventory has been used by Streveler, Miller, and Steif [32]. Equally important, the dynamics-concept-inventory has been researched by other groups including Evans, Gray, and Self [9].

\section{Dynamics Concept Inventory (DCI)}

The Dynamics Concept Inventory (DCI) is typically given as a pre- and post-course assessment tool of important dynamics concepts, providing research data which can be analyzed to determine student conceptual change through the activities and classes. The DCI contains 27 multiplechoice questions covering 11 topics that are found to be important to the study of rigid body dynamics and additional concepts from particle dynamics. Such topics include velocity, acceleration, force, energy, friction, and inertia (each topic is asked on the test by way of multiple questions). Using the Delphi process, a team of professors involved in the American Society of Engineering Education (ASEE) created and implemented the DCI around 2003 [9]. The DCI has been tested and revised to show it is both reliable and valid [9]. Our research group uses the Dynamics Concept Inventory extensively for each developed activity. 
The next section is a description of various teaching styles: traditional teaching, active learning, inductive learning, interactive engagement in physics, and model-eliciting and inquiry-based learning activities. All of these seek to improve student conceptual understanding, among other outcomes, by way of different implementation methods.

\section{Traditional Teaching}

In traditional teaching, instructors convey course material through lecturing and solve example problems in front of the class. Students absorb the material by watching the presentations, listening to the professor speak, writing notes, and working example problems. The teacher goes over several example problems for each concept or chapter in the book, and may provide solution strategies or solution patterns. Some benefits to traditional lecture are covering a great length of course material in the limited schedule time of a course.

A study of eleven physics education sources in the U.S. remarked on deficiencies of traditional instruction [34]. Such findings include: "facility in solving standard quantitative problems is not an adequate criterion for functional understanding, a coherent conceptual framework is not typically an outcome of traditional instruction, and teaching by telling is an ineffective mode of instruction for most students" [34]. These remarks imply that traditional instruction is not an effective method for developing students' conceptual understanding.

In conventional mathematical modeling the models are presented to students, practice problems are provided for applying the models, and then if time permits, strategies are taught for adapting the models to new problem situations [9]. From research on mathematical problem solving over the past 30 years, Lesh and Zawojewski [15] report on various reviews of empirical studies that suggest this conventional approach does not necessarily lead to improved mathematical problemsolving performance. Thus methods of teaching conventional mathematical models could be improved to produce better student problem solving performance. 
Traditional teaching is being contrasted with new teaching methods in education, such as active learning, inductive learning, and inquiry learning.

\section{Active Learning}

A different teaching style from traditional instructional is active learning, in which students learn by engaging the course material actively rather than passively. According to Dr. Michael Prince, the core elements of active learning are introducing student activity into traditional lecture and promoting student engagement [21]. Some ways in which the teacher can implement active learning during class are by having students review their notes, write down points of confusion, make predictions and then view or actively interact with a demonstration or simulation, work together on problems, take an ungraded quiz, and engage in think-pair-share activities. Evidence supporting the successful implementation of these strategies is prevalent in the literature. Ruhl et al. show significant results from having students pause during lecture for a few minutes and have students discuss in pairs and review their notes [26]. The results show "students taught with the pause procedure did significantly better on the free-recall quizzes and the comprehensive test" (comparing two class groups in two different semesters).

Another research group, Laws et al., examined physics students in a study of U.S. universities and noted changes in conceptual understanding between using traditional instruction and active learning methods (the study measured student understanding when they leave the course and if they understand the Newtonian viewpoint). As shown in Figure 1, Laws et al. [14] show that using inquiry-based active learning instruction dramatically increases student performance on questions relating to force, acceleration and velocity. This evidence gives credence to increasing student learning by using active instruction 


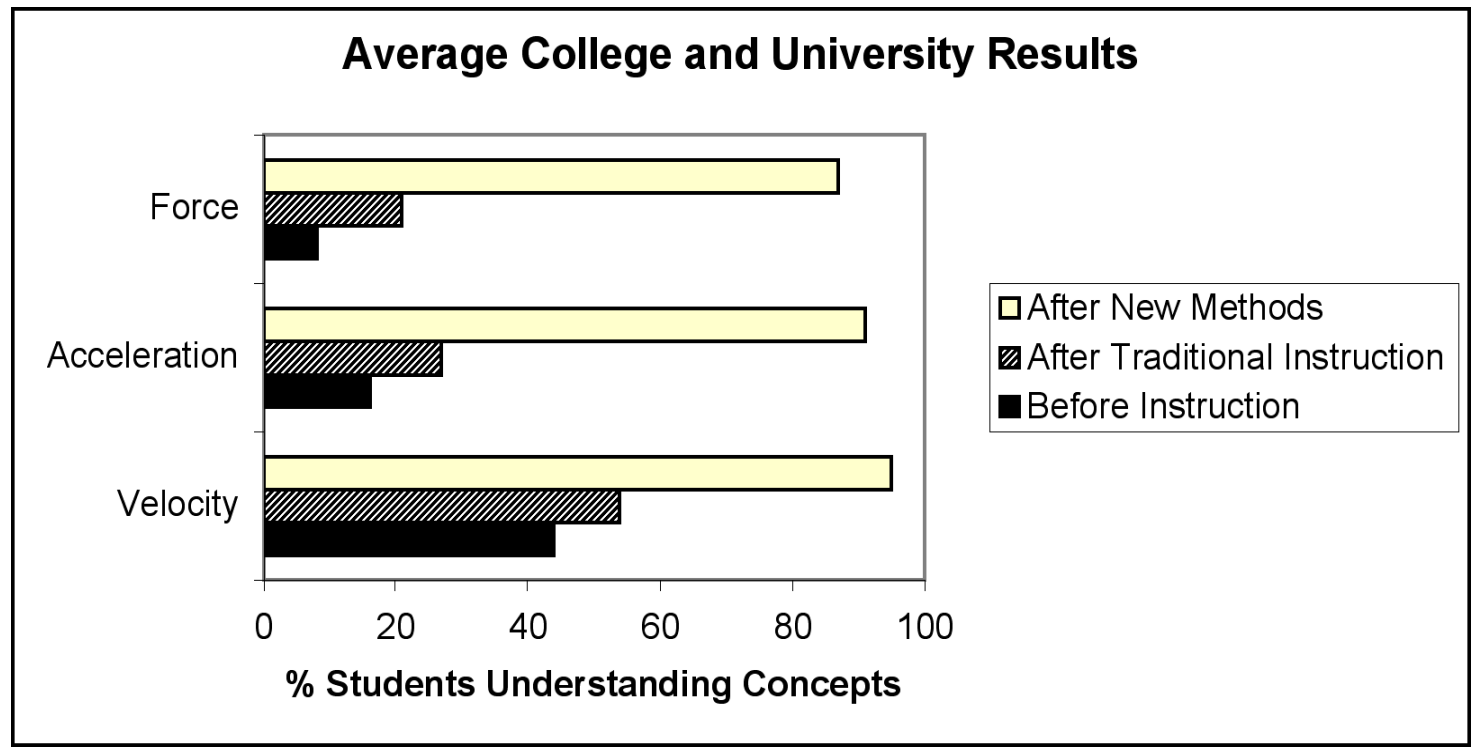

Figure 1. Active-engagement vs. traditional instruction for improving students' conceptual understanding of basic physics concepts (taken from Laws et al.,[14]). Traditional instruction led to a gain of student's conceptual knowledge by 5 to $15 \%$, while active learning results in up to $90 \%$ of students understanding concepts.

Another way to examine teaching methods is to characterize them as inductive or deductive.

\section{Inductive and Deductive Teaching}

Inductive and deductive are two types of learning and teaching which characterize the intended teaching direction of addressing course material. Inductive learning is defined as going from specific contexts to a general concept or theory. For example, the general course concepts, skills, and problem solving methods are examined by the students in the contexts of specific problems, with varying guidance from the instructor. The instructor presents some specific examples in engineering to help students see why they need to learn the material.

On the other hand, deductive learning strives to teach general concepts at the outset and then apply the knowledge to specific problems afterwards. For example, students are taught the work energy principle initially and then given example problems to work through and learn the boundaries and advantages of a given principle. Traditional instruction exemplifies deductive reasoning (starting with general concepts and ending up at specific problems). 
Prince states "while the strength of the evidence varies from one method to another, inductive methods are consistently found to be at least equal to, and in general more effective than, traditional deductive methods for achieving a broad range of learning outcomes" [23]. As a note, there is no pure induction or deduction method and moreover it can be healthy to use a combination of both modes of instruction.

Inductive learning can be implemented in a variety of forms, but here we focus on two. Inductive learning is found in: a) inquiry-based learning activities where students follow guided experiments to uncover general concepts and b) model-eliciting activities, where students are given a specific problem from a client and asked to generate memos and analysis and thus learn general concepts.

\section{Interactive Engagement in Physics}

In the physics community, a study on interactive-engagement vs. traditional lecture has been conducted by Hake to test student understanding in introductory physics classes in high school, college, and university [10]. In a survey of over 6000 students, results from the Halloun-Hestenes Mechanical Diagnostic test, Force Concept Inventory, and Problem Solving Mechanics Baseline test show a normalized gain of $0.23 \pm 0.04$ for traditional methods and $0.48 \pm 0.14$ for Interactive Engagement ${ }^{1}$ methods. His results strongly suggest that classroom use of interactive engagement (IE) methods can increase mechanics course effectiveness in both conceptual understanding and problem-solving well beyond that achieved with traditional (T) methods [11]. For more in depth details of the study and statistical results see the Hake reference [10].

\footnotetext{
${ }^{1}$ IE used Collaborative Peer Instruction; Computer Based Laboratories; Concept Tests; Socratic Dialogue Inducing Labs; Overview Case Study and Active Learning Problem Sets; Modeling; and research-based text or no text.
} 
Active learning has been shown, in some examples above, to be more effective and to better convey conceptual understanding to students than traditional instruction. Building from educational research, the research team is using active learning methods to teach dynamics concepts. Students can utilize active learning through inquiry-based learning and model-eliciting activities, which will be described next.

\section{Inquiry-Based Learning Activity (IBLA)}

The purpose of an Inquiry-Based Learning Activity is to help students learn through inquiry and engagement by having reality act as the 'authority' instead of just the word of the professor. The professor can tell the students why something happens but this may not be as effective as letting the results of the physical experiment communicate the information to the learner. The IBLA method calls for students to make a prediction of a physical situation followed by witnessing the result and reaching conclusions - similar to the scientific method with making a hypothesis followed by experimentation. The students run their own experiments and engage in the learning process during class and lab time, and thus develop conceptual understanding of the appropriate topics.

Although the exact definition of inquiry-based instruction varies somewhat between different investigators, we will use the defining features offered by Laws et al.[14] and highlighted by Prince and Vigeant - "students pose and answer questions through physical experience and direct observation rather than by listening to lectures or following a highly prescribed laboratory experience"[22]. More specifically, the elements for IBLAs are shown in the table below. 
Table 1.Elements of Inquiry-Based Activity Modules [14]
(a) Use peer instruction and collaborative work
(b) Use activity-based guided-inquiry curricular materials
(c) Use a learning cycle beginning with predictions
(d) Emphasize conceptual understanding
(e) Let the physical world be the authority
(f) Evaluate student understanding
(g) Make appropriate use of technology
(h) Begin with the specific and move to the general

In this research, student teams make use of the IBLA method by first making a prediction of a physical experiment, then running the experiment and filling out a worksheet. Their predictions and worksheet are reviewed by the research team to evaluate student understanding through the course of the activity. Because students run the experiment themselves and observe the result, the physical world acts as the 'authority', rather than the professor.

Evidence for IBLAs is found in literature. In the realm of engineering, Prince and Vigeant investigate IBLAs for teaching undergraduate heat transfer. Their data show improvements in student's conceptual understanding using inquiry-based activities over traditional instruction gains. These results were found to be comparable or better than those found for similar analysis of IBLAs with undergraduate physics students [22]. In study a by Thacker et al. students in an introductory inquiry-based physics class were compared to traditional introductory course students on a qualitative and quantitative problem. The students in the inquiry-based physics course performed significantly better on the two problems used [33].

\section{Model-Eliciting Activity (MEA)}

Students engage in model-eliciting activities in order to develop conceptual understanding by working in teams to construct, test, and refine a conceptual model which is later given to a client. 
Model-eliciting activities (MEAs) are "open ended, realistic, client-driven problems that require the creation or adaption of a mathematical model for a given situation" [9]. MEAs were developed in the mathematics community as a way to promote model creation and elicit student thought processes. In response to an open ended prompt, student teams analyze a problem and submit a model to the client to solve a specified need, which in turn displays their thinking processes. Students are required to document their thought processes - typically though a memo to the client. This submission reflects student thought processes and ways of thinking rather than just a simple numerical response. MEAs match six main principles which make them distinct from IBLAs and textbook problem learning. The six principles are displayed below:

- Model Creation - Students create a model of a realistic problem using engineering quantities and other prior knowledge. They create "symbolic descriptions of meaningful situations" [16] as opposed to decoding symbols found in textbook problems.

- Reality - the MEA presents an authentic engineering context to the students: for example, a patient needs recommendations for safe athletic motion or a Sri Lanka police department needs assistance with vehicle-accident-reconstruction. The real context motivates the student to recognize the need for a solution [16]. These real world scenarios are examples of what students might face later in their engineering career.

- Self-assessment - MEAs serve students by providing engineering context and an opportunity to iterate their own problem-solving thought processes. In order for students to improve and refine the model they create, they must be able to tell when their model is sufficient, or to judge the validity of their results. Therefore the MEA must set up students to self-assess the progress and usefulness of their results [16].

- Model Documentation - MEAs can create a "window on many facets of student learning. Instructors and researchers can attend to: students' conceptual understanding of mathematics, science, engineering, and technology; students' development of problem- 
solving interactions; technical communication; or the relationships among these," p 34, [35]. Model-eliciting activities elicit and reveal thoughts of the students for the teacher to witness, where the teacher can glance into the problem solving process of the student and pick up their reasoning or their constructs. MEAs not only document the problem solving process but "support the productivity of ongoing learning or problem solving experiences" of the students [16].

- Generalizability - One criterion is that the solution should be able to address a wide range of problems that may arise rather than just one specific problem. The solution should extend to future situations and future contexts. This allows students to refine and extend their previous conceptual knowledge [16].

- Effective Prototype - Students build an effective prototype solution, or develop concepts or tools, which can be used as a template for future work. Additionally, the activity must use important dynamics concepts.

\section{Overall Purpose of Research Project}

The main purpose of this research is to implement and assess both inquiry-based learning and model-eliciting activities to teach a selection of dynamics concepts in a meaningful way and build correct conceptual understanding. The concepts that the activities target include: workenergy, Newton's second law, angular and linear accelerations, the effects of inertia, gyroscopic motion, and work-energy and impulse momentum. The research team tests the applicability and usefulness of the two methods and improves their implementation with each revision cycle

This research project has also been motivated by National Science Foundation grants. The IBLA research has prompted work in developing and testing new IBLAs to teach important concepts, as well as to assess the effectiveness of these interventions [27]. The MEA research discussed here seeks to develop a new activity, which resulted in the gait analysis activity, as well as continue 
using previous MEAs. This research can also study students' problem solving strategies and extend their use to reasoning and problem solving [36].

With the motivation for active learning methods and specific purpose stated, we now begin to describe the implementation of the research in two classes offered at Cal Poly, following with the results and discussion after each activity section.

\section{Dynamics Content}

Two dynamics classes are taught at CSU Cal Poly: Engineering Dynamics (required course for most engineering majors) and Intermediate Dynamics (required for mechanical engineers).

Engineering (or beginner) Dynamics involves "analysis of motions of particles and rigid bodies encountered in engineering; velocity, acceleration, relative motion, work, energy, impulse, and momentum; further development of mathematical modeling and problem solving; vector mathematics where appropriate" [20]. Activities such as mass-pulley, rolling objects, spools, and vehicle-accident reconstruction teach important class concepts in introductory dynamics.

Intermediate Dynamics involves "additional analysis of planar motion of rigid bodies with particular attention to the kinematics of mechanisms; rotating reference frames; introduction to three dimensional dynamics; dynamic simulation of mechanisms" [20]. Activities such as gyroscope and gait lab are utilized in intermediate dynamics to cover relevant concepts.

The activity and concepts chosen for implementation in IBLA or MEAs are ones that are important and difficult for the students, which can be seen in the following Table 2 . 
Table 2. Activities and concepts implemented

\begin{tabular}{|c|c|c|}
\hline Activity & Type & Desired conceptual learning \\
\hline Mass-Pulley & IBLA & Newton's $2^{\text {nd }}$, the effects of inertia \\
\hline Spool & IBLA & Newton $2^{\text {nd }}$ law, FBD, friction \\
\hline Rolling Objects & IBLA & MOI, Work-energy, \\
\hline Gyroscope & IBLA & $\begin{array}{c}\text { Gyroscopic motion, } \\
\text { angular momentum }\end{array}$ \\
\hline Gait lab & MEA & $\begin{array}{c}\text { FBD, Rigid Body Kinematics/Kinetics, } \\
\text { Newton's } 2^{\text {nd }}, \\
\text { linear and angular acceleration }\end{array}$ \\
\hline $\begin{array}{c}\text { Vehicle accident } \\
\text { reconstruct }\end{array}$ & MEA & Work-energy, impulse-momentum \\
\hline
\end{tabular}




\section{IBLA ACTIVITIES}

Inquiry-Based Learning Activities have students make a prediction before running an experiment involving multiple cases. Students work in teams to learn from the physical experiment and build their conceptual understanding. The Inquiry-Based Learning activities for the 2012-13 year were mass-pulley, spools, rolling objects, and gyroscopes; occurring in either Engineering Dynamics or Intermediate Dynamics.

\section{2: MASS-PULLEY IBLA}

In this activity, students experimented with basic Atwood machines, depicted in Figure 2. Two pulley systems were attached to a single rod so they could be held by a single student and the motion behavior can be compared by a side-by-side visual "race." Two mass-pulley systems raced against each other when both

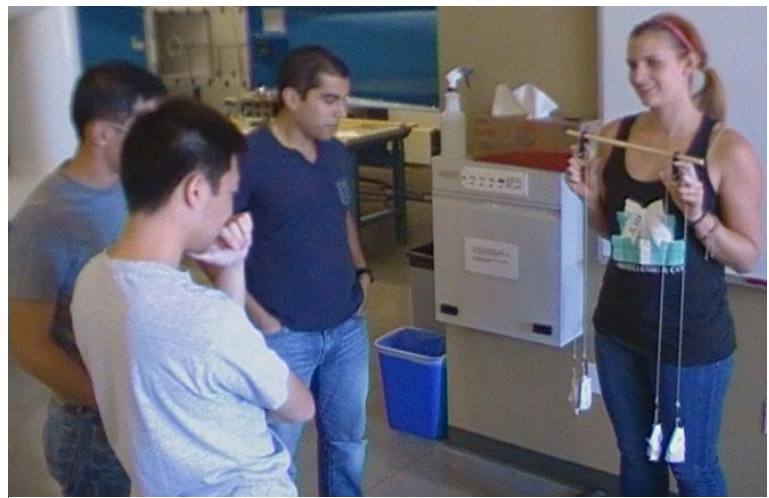

Figure 2. Mass-Pulley Inquiry activity in action

systems were released from a raised position and gravity acted on the masses to propel the system into action. The goal of this activity was for students to investigate how a system accelerates depending on the net force applied to it and its overall system mass. For each system, the heavier mass accelerated downward while the lighter accelerated upward. The pulley inertia, rope mass, and friction were considered negligible.

This activity was initially run as a class demonstration in Intermediate Dynamics in Spring 2013 and as an IBLA group activity in Introductory Dynamics in Spring 2013. With the demonstration class, the professor performed one scenario using 3, 4, 9, and 10 ounce weights and discussed the results afterwards. 


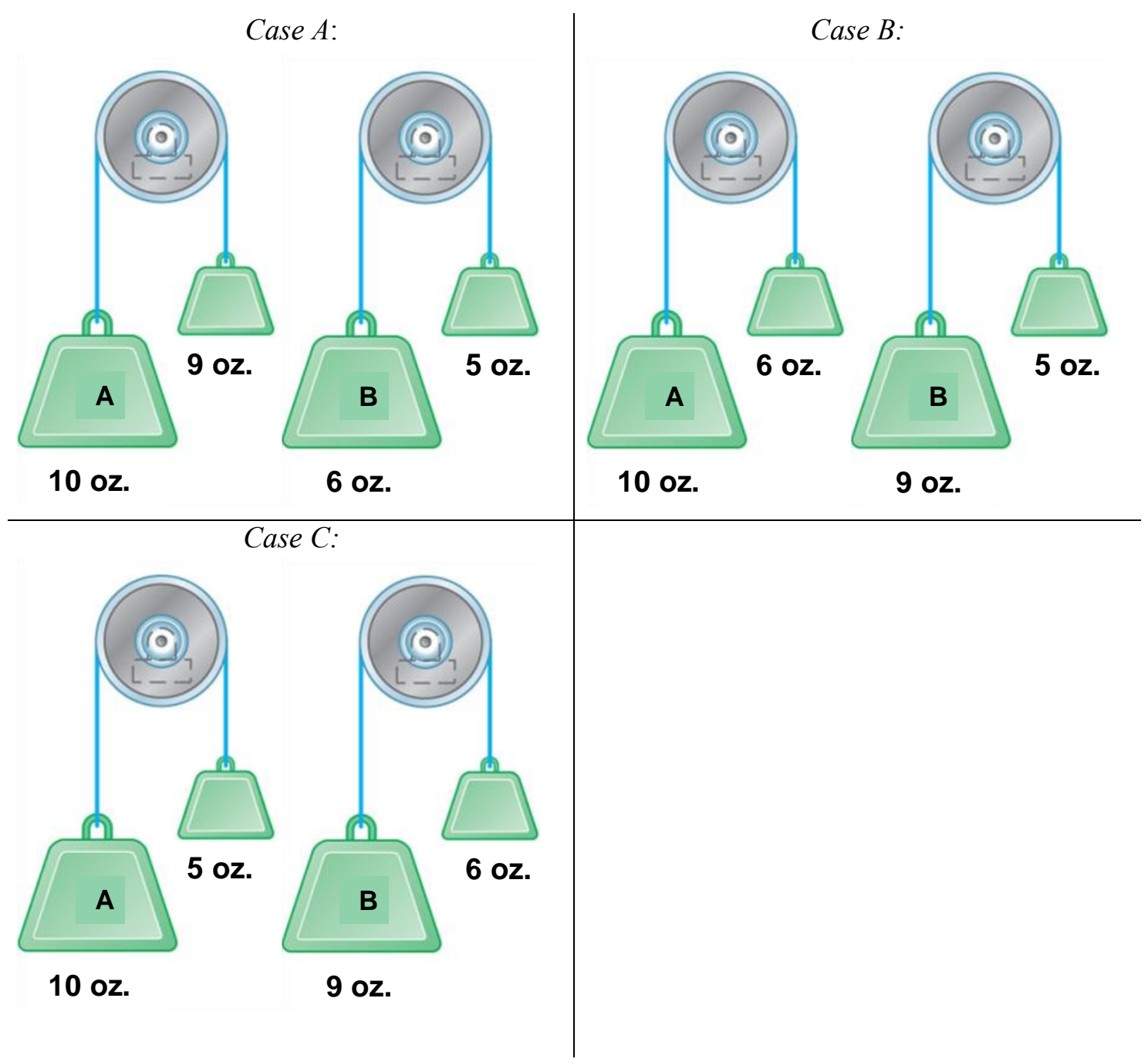

Figure 3. Mass-Pulley IBLA setup for each case.

Alternatively, students in the inquiry activity (IBLA) worked in teams through three scenarios with permutations of 5, 6, 9, and 10 ounce weights (Figure 3). Case A featured two systems where the net force was the same, but total mass was different-10-9 vs. 6-5 ounce weights. Case B featured two systems where the total mass is the same, but the net force is different-105 vs. 9-6 weights. Finally, Case C featured two systems with the same net force, but different total mass- 10-6 vs. 9-5 weights.

These different cases helped the students to explore the force and inertia concepts from various viewpoints. Following each scenario, the professor spoke to clarify concepts, mediated a class discussion, stated his logic for the system behavior, and answered student questions. 
To analyze which mass-system accelerated faster than the neighboring system, students analyzed the net force and net mass of the system. This activity investigated the application of Newton's second law $\left(\overrightarrow{\sum F}=m \cdot \vec{a}\right)$, including the DCI concept of effect of inertia, on the mass-pulley systems. For example, two systems with same net applied force do not accelerate with the same magnitude because they both have different total system mass.

Conceptual understanding of acceleration and inertia was assessed through various items. Students were first assessed with a pre-activity DCI test 1-2 weeks before the IBLA. Specifically, question 13 asks students to predict the acceleration of systems very similar to the Atwood machines used in the IBLA (Figure 4). In addition, students filled out a worksheet on the activity day, addressing the three cases. The

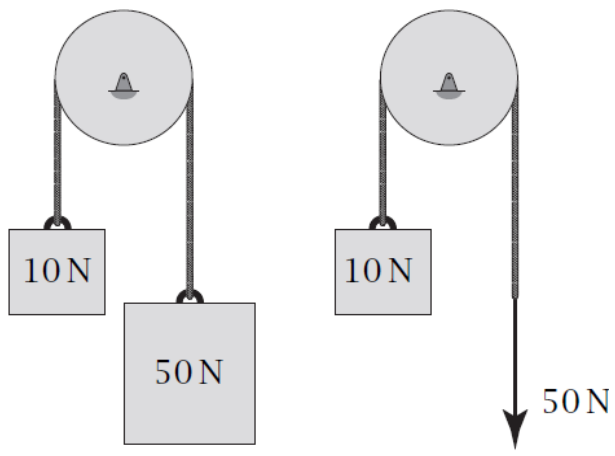

Figure 4. DCI Question \#13: Which $10 \mathrm{~N}$ block will have a larger acceleration?

worksheet had students write predictions, state observations and explain the behavior using principles of dynamics (worksheet in Appendix A). Later students took a quiz to see if they really understood the concept and even later faced a midterm question which tested their transfer ability. The midterm question consisted of two see-saws, with a weight force on one see-saw, and a massless force on the other see-saw. The question asked which see-saw would accelerate a gymnast/cat more (Figure 5). 
6. You want to launch a 10-lb cat into the air because it is making Dr Self sneeze. You use a super-dooper lightweight carbon see-saw, and wonder how two different designs will work. Design A: you put a $100 \mathrm{lb}$ weight on the end, and will release the system from rest. Design B: while the system is at rest, you will apply a constant $100 \mathrm{lb}$ force to the see-saw. Which of the following is true immediately after the systems starts from rest? Will the cat land on his feet?

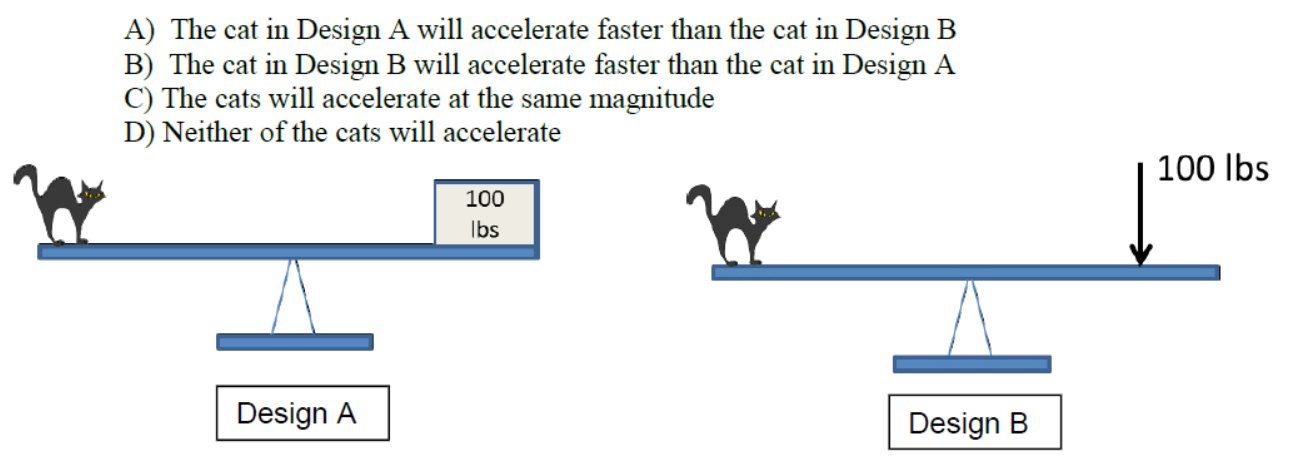

Figure 5. Midterm Problem where students transfer the concepts of Newtons $2^{\text {nd }}$ Law to the case of a seesaw, with a weight force on case ' $A$ ' and a mass-less force on case ' $\mathrm{B}$ '.

To further gauge understanding students took the Post DCI at the end of the quarter. Comments and suggestions were received when students filled out a subjective survey, also at the end of the quarter.

As a reminder, the activity was run for Introductory Dynamics as IBLA group activity and for Intermediate Dynamics as a class demonstration. The demonstration and inquiry activity results cannot be directly compared because they were for different class levels. In addition, the introductory class consisted of a range of engineering majors while the Intermediate class consisted of almost entirely mechanical engineers.

\section{Mass-Pulley Results (IBLA)}

The scores from the DCI of the Introductory Dynamics students are shown in Table 3. The IBLA was implemented in spring 2013, while non-IBLA classes were held in previous quarters at Cal Poly and also at a small and large public university. 
Table 3. DCI dealing with Mass-Pulley Concept, for multiple Introductory Dynamics classes

\begin{tabular}{|l|c|c|c|c|}
\hline \multicolumn{1}{|c|}{ Class Label } & $\begin{array}{c}\text { DCI } \\
\text { Pre class } \\
\text { Question 13 }\end{array}$ & $\begin{array}{c}\text { DCI } \\
\text { Post class } \\
\text { Question 13 }\end{array}$ & $\begin{array}{c}\text { Normalized gain } \\
\text { Pre to Post DCI } \\
\text { Question 13 }\end{array}$ & IBLA \\
\hline Large Public Univ., [9] & $4.6 \%$ & $56.1 \%$ & 0.539 & No \\
\hline Small Public Univ., [9] & $5.5 \%$ & $36.1 \%$ & 0.324 & No \\
\hline Fall 2012 BPS & $\begin{array}{l}12.2 \% \\
(\mathrm{n}=99)\end{array}$ & $\begin{array}{c}84.95 \% \\
(\mathrm{n}=91)\end{array}$ & 0.830 & No \\
\hline Fall 2012 JW & $\begin{array}{c}15.05 \% \\
(\mathrm{n}=86)\end{array}$ & $\begin{array}{c}89.16 \% \\
(\mathrm{n}=81)\end{array}$ & 0.872 & No \\
\hline $\begin{array}{l}\text { Winter 2013 JW } \\
\text { (intermediate) }\end{array}$ & $\begin{array}{l}22.2 \% \\
(\mathrm{n}=27)\end{array}$ & $\begin{array}{l}89.3 \% \\
(\mathrm{n}=22)\end{array}$ & 0.862 & Yes \\
\hline Spring 2013 BPS & $\begin{array}{l}14.8 \% \\
(\mathrm{n}=27)\end{array}$ & $\begin{array}{l}93.8 \% \\
(\mathrm{n}=32)\end{array}$ & 0.927 & \\
\hline
\end{tabular}

The spring '13 class, featuring the implementation of inquiry based learning, yielded the largest normalized gain compared to the previous three fall classes. The winter '13 class was an intermediate dynamics course, where students had already taken one class in dynamics. The low Pre-DCI score showed students had a weak understanding of the concept of the effect of inertia at the beginning of the course, and set a baseline of student understanding. The effect of inertia details how a system linearly accelerates depending upon its mass. The Post-DCI showed that students' understanding of the effect of inertia improved (students scored as much as $93.8 \%$ correct on Q13) ${ }^{3}$. The DCI scores of the IBLA class were not much greater than scores of the non-IBLA classes, but were substantially higher than those reported at other universities.

The worksheets used during the activity provided a place for team predictions, where students could display their results and explain their reasoning to the instructor. Student teams made predictions on the activity worksheet to answer the prompt: "Consider the masses $A$ and $B$ with weight as shown (Figure 3). What do you predict about the accelerations of the masses if they are

\footnotetext{
${ }^{2}$ Normalized gain is calculated as the change in scores divided by the maximum change possible.

${ }^{3}$ There was a larger quantity of students who took the post-DCI item than the pre-DCI item.
} 
released from rest?" Next, results are presented from a worksheet filled out by each team during the activity.

Table 4. Mass-Pulley Results from team inquiry, Engineering Dynamics class

\begin{tabular}{|c|}
\hline $\begin{array}{c}\text { Team predictions on worksheet, } \\
\text { correct } \%\end{array}$ \\
\hline Case A: $63.6 \%$ \\
Case B: $90.9 \%$ \\
Case C: $96.9 \%$ \\
$(\mathrm{n}=33)$ \\
\hline
\end{tabular}

Looking at the team worksheet, the students' predictions of the behavior of the mass-pulley system improved from case A to B and from B to C. This might indicate student conceptual understanding grew through the activity and thus they were able to make correct predictions when they figured out the significant concept and were able to apply it. Another reason for prediction improvement could be due to the social interaction amongst teammates and the bolstering of students' confidence.

Next, we assess the team reasoning patterns through the activity. Out of the nine teams:

- Four teams held consistent reasoning through the activity and did every case correctly.

- Four teams changed their reasoning between case B and C, and reached the correct understanding on case $\mathrm{C}$.

One team explained every case incorrectly, with the consistent reasoning. (This team drew the FBD for the heavier block and stated $\sum F=$ tension $-\mathrm{m}_{\text {heavy* }} \mathrm{g}$ for the heavier block, where their tension was 'the mass of the lighter block'). In Case A, students used different reasoning of dynamics principles to compare the behavior of the falling masses. Students mentioned Newton's 2nd Law ( $\mathrm{F}=\mathrm{ma})$ and gave such responses as "the ratio of masses in the second case [pulley system] is more prominent than the first," "dividing by a smaller mass will give a larger acceleration," and "the difference in mass but same net force will yield one to accelerate greater 
than the other." Following the class discussion, the correct conclusion was reached for Case A on a class level. This is best exemplified by one group's response: “The net force is 1oz, but higher system net mass leads to a lower net acceleration."

In Case B, students used various explanations of dynamics principles to relate the behavior between the neighboring pulley systems. Students responded with such things as "the bigger ratio between masses in the first system will yield a larger acceleration." The ratio of masses in a pulley system does positively correlate with block acceleration as seen in the equations below.

$$
\begin{aligned}
\vec{F}_{\text {net }} & =m \times \vec{a} \\
\left(w_{\text {heavy }}-w_{\text {light }}\right) & =\frac{\left(w_{\text {heavy }}+w_{\text {light }}\right)}{g} \vec{a} \\
\vec{a} & =\frac{g\left(w_{\text {heavy }}-w_{\text {light }}\right)}{\left(w_{\text {heavy }}+w_{\text {light }}\right)} \\
\vec{a} & =\frac{g\left(w_{\text {heavy }} / w_{\text {light }}-1\right)}{\left({ }^{w_{\text {heavy }}} / w_{\text {light }}+1\right)}
\end{aligned}
$$

Thus, as the ratio $w_{\text {heavy }} / w_{\text {light }}$ or $m_{\text {heavy }} / m_{\text {light }}$ increases, so does the acceleration.

(Equation (4) is plotted). 


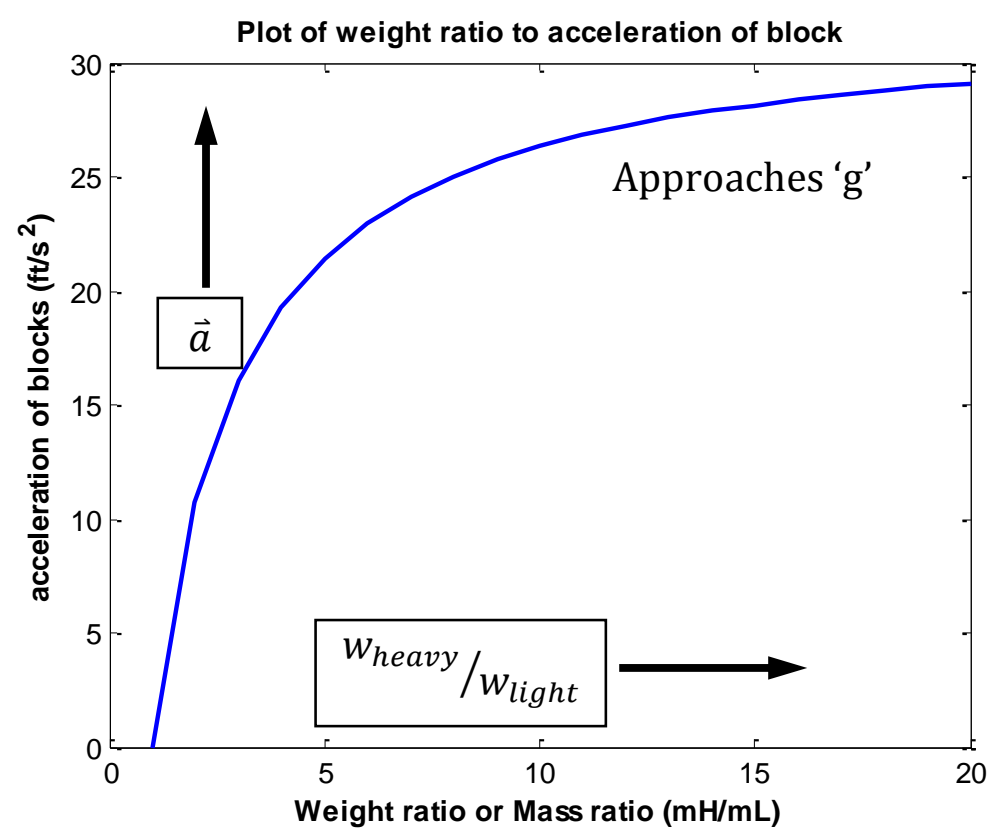

Figure 6. Plot of weight ratios to acceleration of block

Other explanations include: "the net force is bigger for block A, but both systems have the same mass so block A will go faster," and that Newton's second law can be applied. Students were able to reach the conclusion following class discussion that there is the same net mass, but lower net force, so different net acceleration.

In Case $\mathrm{C}$, students continued to explain the behavior correctly and in addition make more accurate predictions. Students mentioned case $\mathrm{C}$ was similar to case $\mathrm{A}$. They stated there was the same net force acting on the two pulley systems and they observed the masses of the two systems were different. The students again made use of Newton's second law.

Students revealed their misconceptions such as not identifying the correct total mass of the system. The reasoning of the one group who did the cases wrong stated that tension in the rope is equal in magnitude to the opposite block's weight (which is not true for accelerating systems). In the future, the instructors can address these specific misconceptions during the class discussion or in office hours. 
Students were engaged in the activity and had meaningful conversations with group members when deducing the responses to the given prompts on each mass-pulley case. Often they would ask each other questions and group members would respond back with their reasoning. For example, one group member realized the correct concept and then proceeded to explain with excitement to his team members.

A few weeks following the activity students applied the conceptual understanding from the activity to a new context on the midterm. The midterm problem was a transfer problem of a seesaw setup (instead of a pulley setup) with a weight force acting on one system and a weight-less force acting on another system.

Table 5. Midterm Results

\begin{tabular}{|l|}
\hline $\begin{array}{l}\text { Midterm Transfer } \\
\text { question }\end{array}$ \\
\hline $\begin{array}{c}\text { Q6: } 88.24 \% \text { correct } \\
(\mathrm{n}=34)\end{array}$ \\
\hline
\end{tabular}

The midterm results displayed conceptual improvement $(88.24 \%$ of students answered the question correctly).

A subjective survey was given at the end of the course to elicit feedback and garner suggestions for improvement (Table 6). The response scale used was a Likert Scale: 1=strongly disagree | $2=$ disagree $\mid 3=$ neither agree nor disagree $\mid 4=$ agree $\mid 5=$ strongly agree. The survey showed the activity was interesting, helpful to learning, and should be continued in the future (Table 6). Students also commented that a demonstration would be neutrally effective in learning the concepts as compared to a group activity (scoring 3 out of 5). Students may prefer the demonstration due to it taking less time to perform or students may prefer to avoid the effort of group work. One such student commented: "The pulley/weight activity was way too sensitive to learn from. It was really hard for me to tell what was clearly going on when I was doing the 
activity because the results changed so often. I would have preferred to have just been taught the material from Dr. Self himself." On another survey question, most students stated they trusted the results of the activity, while a small number were still skeptical (citing challenges with the experimental setup).

Table 6. Mass-Pulley IBLA Survey Results

\begin{tabular}{|c|c|c|}
\hline $\begin{array}{l}\text { The mass-pulley lab was } \\
\text { interesting and motivating: } \\
\mathbf{4 . 1} / \mathbf{5 . 0}\end{array}$ & $\begin{array}{l}\text { The mass-pulley lab } \\
\text { helped me learn about } \\
\text { F = ma: } \\
\text { 3.9 /5.0 }\end{array}$ & $\begin{array}{l}\text { You should do the mass- } \\
\text { pulley lab in future sections } \\
\text { of the course: } \\
\mathbf{4 . 1} \text { / } \mathbf{5 . 0}\end{array}$ \\
\hline $\begin{array}{l}\text { Having the professor do a } \\
\text { pulley demo at the front of } \\
\text { the room would be just as } \\
\text { effective as the group } \\
\text { activity: } \\
\mathbf{3 . 0} / \mathbf{5 . 0}\end{array}$ & \multicolumn{2}{|c|}{$\begin{array}{l}\text { Did you trust the results of the pulley lab? } \\
\text { - Yes - } \mathbf{8 7 \%} \\
\text { - A bit skeptical - } \mathbf{1 3 \%} \text {, with responses: } \\
\text {-"Due to masses hitting each other" } \\
\text { - "Race result seemed too close to call" }\end{array}$} \\
\hline
\end{tabular}

\section{Mass-Pulley Results (Demonstration)}

Alternatively, the mass-pulley activity was shown as a class demonstration with only one scenario in Intermediate Dynamics, which is typically taken 1-3 quarters after Engineering Dynamics. The scenario prompt was similar to scenario A for the introduction class, but with different values for the masses. This class is comprised of over $85 \%$ mechanical engineering students, enrolled in two class sections. Similar assessments were given to the students. A DCI pre-activity was given at the beginning of the course, while a post-activity DCI was given at the end of the 10 week quarter. Additional assessments were given: and a personal prediction and activity worksheet. Students made individual predictions for Case A only, via Poll-Everywhere using cell-phones (Polleverywhere.com). A few days later students took a post-activity quiz individually, where they ranked four cases of different masses and forces. The following midterm posed a transfer question involving a gymnast on a see-saw, with a weighted force and a mass-less force. The results can be seen in the following table. 
Table 7. Mass-Pulley demonstration results for Intermediate Class

\begin{tabular}{|c|c|c|c|c|c|}
\hline $\begin{array}{c}\text { DCI } \\
\text { Q13 }\end{array}$ & $\begin{array}{c}\text { Individual- } \\
\text { prediction Poll- } \\
\text { everywhere }\end{array}$ & $\begin{array}{c}\text { Team } \\
\text { predictions, } \\
\text { Activity } \\
\text { worksheet }\end{array}$ & $\begin{array}{c}\text { Post Class } \\
\text { Quiz }\end{array}$ & $\begin{array}{c}\text { Midterm } \\
\text { (Gymnast } \\
\text { Transfer } \\
\text { question })\end{array}$ & $\begin{array}{c}\text { DCI } \\
\text { Post Activity } \\
\text { Q13 }\end{array}$ \\
\hline $\begin{array}{c}43.3 \% \text { correct } \\
(\mathrm{n}=67)\end{array}$ & $\begin{array}{c}50 \% \text { Correct } \\
(\mathrm{n}=68)\end{array}$ & $\begin{array}{c}75 \% \text { Correct } \\
(\mathrm{n}=68)\end{array}$ & $\begin{array}{c}60 \% \text { received } \\
\text { full score } \\
(\mathrm{n}=55)\end{array}$ & $\begin{array}{c}72 \% \text { chose } \\
\text { correct answer } \\
(\mathrm{n}=68)\end{array}$ & $\begin{array}{c}87.3 \% \text { correct } \\
(\mathrm{n}=71)\end{array}$ \\
\hline
\end{tabular}

From the table, the Pre-DCI scores, which offer a snapshot into student thinking, indicated a medium range of understanding for the concepts (students answering 43\% correct on Q13). On the activity prediction sheet, the correct answer was predicted by individuals at $50 \%$, and in teams at $75 \%$ correct. Thus team interaction could lead to improved understanding of the system acceleration concept.

Teams filled out worksheets with experiment results and explanations. Most teams correctly observed that the net force is equal in the neighboring systems, but that system B has less total mass so it accelerates faster. Some groups reasoned through the activity by drawing FBDs and KDs. Other teams stated "the greater the percent difference between the masses, the greater the acceleration" and "the ratio of the masses is greater so the force from $3 \mathrm{oz}$. counteracts the $4 \mathrm{oz}$. less." These statement examples represent medium understanding, which could be improved to a higher state of understanding. One misconception that was stated was "the tension force is the same for both cases" (when really the tension depends on the acceleration of the blocks; the net force was the same in both systems).

Students were further tested on their conceptual knowledge on a quiz and midterm. Sixty percent of students got a full score on the quiz problem. On the midterm problem students scored an average score of $72 \%$ correct. These low scores from the quiz and midterm signal that students had trouble applying their knowledge to transfer problems involving a massless-constant force. 
Students may have not fully understood these concepts when taking the quiz and midterm. Furthermore, the demonstration used here may be ineffective in relaying these concepts.

\section{Mass-Pulley Discussion}

The mass-pulley activity for the team-inquiry class provided a working environment to learn the concepts through a set of active experiments and class discussion. Students in the inquiry-based learning class showed improvement in recognizing the correct conceptual behavior, answering Q13 with 93\% correct result on the Post-DCI concepts. Likewise, the Demonstration class (Intermediate) scored $87 \%$ on the post-DCI. These students showed significant transfer with their high average score on the midterm problem ( $88 \%$ for beginner class, $72 \%$ for Intermediate class).

Alternatively, the demonstration provided visual behavior of the physical mass-pulley system to the students to teach the concepts. Students improved their conceptual knowledge by witnessing the demonstration and participating in the class discussion. The intermediate class was compromised of sophomore and junior level mechanical engineering students, who experienced these concepts in previous classes. The class who witnessed the demonstration achieved an $87 \%$ average score on Q13 of the Post-DCI question at the end of the quarter, from starting the quarter with a score of $43 \%$ on the Pre-DCI.

This research investigated whether inquiry based learning was an effective method to promote conceptual understanding and whether students in turn were able to apply the concepts to other contexts. The transfer problem sampled students' ability to apply the concepts of net force, system mass, and acceleration to an unfamiliar problem context. Students became comfortable with forces due to weight, but they were less comfortable when dealing with massless forces. The low scores on the transfer problem, for the Intermediate students, suggest the need to revise the hands-on activity to better achieve understanding and thus student transfer. This could be done by adding different cases and challenging their misconceptions, or through added lecture. 
Student responses on the survey were positive concerning the helpfulness and motivation provided by the activity. They also rated the demonstration neutrally—perhaps some students prefer a demonstration style over group activity.

Overall, this inquiry activity adequately promoted conceptual understanding of the force and inertia concepts to dynamics students. The Dynamics Concept Inventory scores reflect student understanding of how inertia affects system behavior, from the beginning to the end of the course. The scores from the interactive activity were not much greater than the results from the demonstration. This activity will continue in the future and improve with subsequent implementations.

\section{Mass Pulley Improvements}

Although the aims of this activity have been properly met, we have identified areas of improvement with the experimental setup. To alleviate student confusion of the race results, we can make all of the results more obvious. This can be achieved by selecting the combination of mass values of each race to produce large differences in accelerations. Likewise, it would greatly improve the IBLA if there was a physical setup to portray system behavior caused by a constant force; this behavior is different than that caused by the force of a falling mass. This might be done with the use of a constant force spring or some other actuator. However this setup is difficult to achieve in practice due to the finicky nature of using constant force springs. In addition, thicker diameter string or rope will be used to alleviate the problem of the rope becoming entangled too easily as seen with struggles with thin fishing line. 


\section{3: SPOOL IBLA}

Students investigated the dynamic behavior of a rolling rigid body in the spool inquiry-based learning activity. A spool with a string wound around the inner radius was rolled to the side when subjected to a string pull at a horizontal or
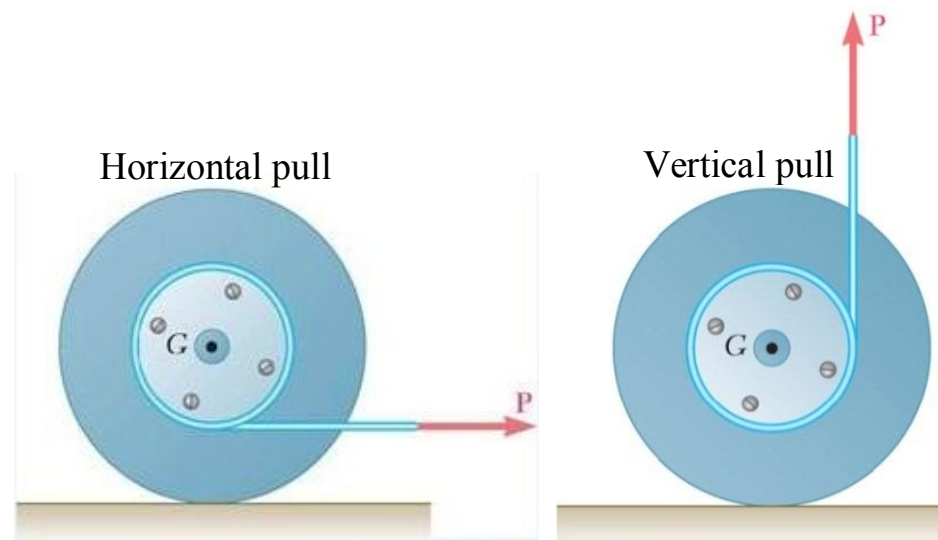

Figure 7. Spool diagram for both cases: horizontal pull (left) and vertical pull (right) [20]

vertical angle. After making predictions about the spool behavior, students experimented in teams and answered question prompts stemming from two pull-scenarios (horizontal and vertical). They were tasked to find which direction the friction force on the bottom of the spool acted and which way the spool would travel. To reason if the spool rolled left or right and determine friction direction, students analyzed the sum of forces and sum of moments about the center of gravity of the spool, as well as drew free body diagrams and kinetic diagrams as reference tools. In the first scenario, students made a prediction and then pulled the rope at a horizontal angle (Figure 7, left image).

Then the professor intervened and discussed the logic behind the rolling behavior for the entire class. This cleared up student confusion and guided the inquiry activity. He drew a free body diagram (FBD) which included forces, and then the kinetic diagram (KD) which contained accelerations multiplied with inertias. Following the professor-led discussion, students continued with the activity and for the second scenario pulled the string at a vertical angle (Figure 7, right image). Again, students made predictions, ran the experiment, discussed in teams (Figure 8), and participated in a professor-led class discussion. 
During the class discussion, students were asked if the spool could spin in place if the pull angle was in between the horizontal and vertical angles, at a 'magic angle'. They were also asked: a) how the magnitude of the static friction force approaches its maximum value before slipping occurs and switches to kinetic

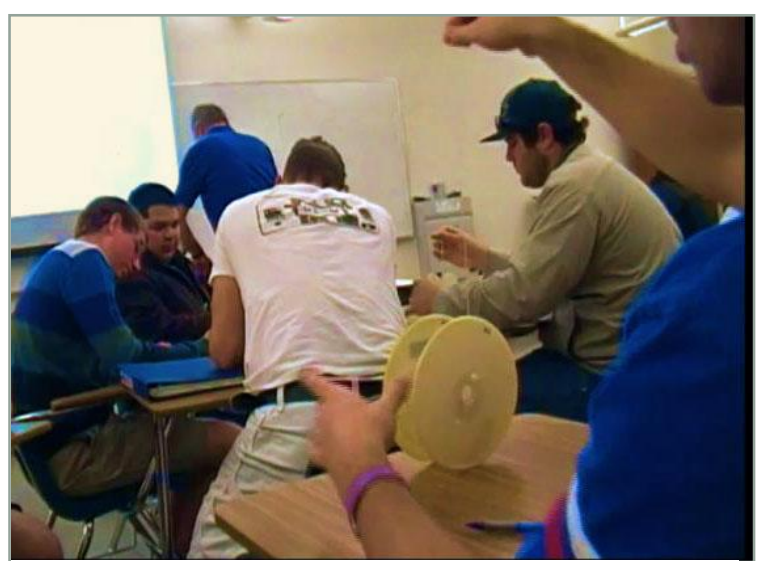

Figure 8. Spool activity is rolling into action! friction and b) what happens when a relatively large pulling force causes the spool to roll with slip.

Students worked to draw FBDs and KDs for the spool object, to correctly identify the friction force direction, and to find the corresponding rolling direction (and linear acceleration). Through the activity students confronted the DCI concept that "the direction of the friction force on a rolling rigid body is not related in a fixed way to the direction of rolling" [9]. For instance, the spool rolled in different directions in the horizontal case and vertical case, yet the friction force in both scenarios acted in the same direction.

The research team assessed students' conceptual understanding of the dynamics of rolling without slip throughout the class term. For initial assessment, students answered a conceptual question involving friction force of an automobile tire, of both front and rear, which rolled without slipping (DCI, Figure 9). The automobile tire problem is related to the Spool activity because in both cases

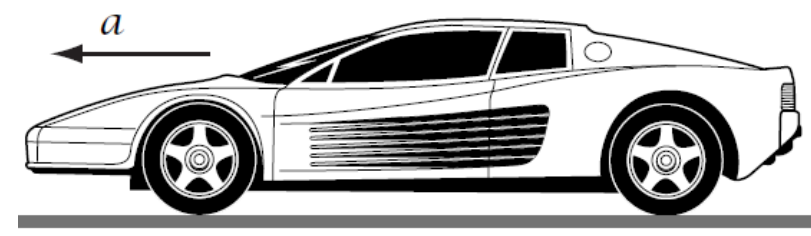
students must make the connection Figure 9. Automobile tire friction question on DCI: Find the friction force direction and expression for the front and rear tires [9].

between rolling direction and friction direction, which are not always pointing in the same direction. The automobile question is an indirect assessment and is more of a transfer problem 
than the spool problem. The automobile question gives the linear acceleration direction to the student, rather than giving the force applied, and asks to find the friction force direction. Students must also reason whether the friction is less than or equal to $\mu_{\mathrm{s}} \mathrm{N}$ or $\mu_{\mathrm{k}} \mathrm{N}$.

The assessments continued throughout the quarter after the initial DCI task. Before the inquiryactivity, students made individual predictions using their cell phones and Poll Everywhere website (www.polleverywhere.com). Next, they discussed the problem with their teammates and made a team prediction. During the activity, students recorded their experimental results on a team worksheet (found in the Appendix B).

Students were given a quiz either before the activity or weeks later to test material understanding ${ }^{4}$. The early implementations used a quiz the day before the activity to get students to think about the problem before coming to class. The later implementations instead held predictions beforehand, and administered a quiz after the activity. For example, the follow on quiz question probed concepts of angular acceleration and friction coefficients, which extended the dynamics concepts in a quantitative problem.

The professor held an intervention mid-activity in the latter three courses, from the experience of the first implementation. For example, after the horizontal pull case, the professor asked why the spool rolled a certain direction and how to reason the friction direction. Students pitched their ideas and discussed as a class. This effort was to make sure all of the students were on the same page and understood the concepts.

In addition, students were tested on what they had learned on an exam question. Weeks after the activity, students were assessed with the same conceptual test (DCI) to record their retention of

${ }^{4}$ The Fall engineering dynamics class used an online, pre-class quiz. The Winter Engineering Dynamics class used a post, paper quiz. The Winter intermediate Dynamics class used a pre-class quiz. The Spring Engineering Dynamics class used a post-class quiz. 
the concepts. Finally, students submitted a survey to give feedback and claim whether the activity was useful, interesting, and rate its importance relative to different class activities.

The spool activity ran during fall, winter, and spring of the 2012-13 year for undergraduate dynamics.

\section{Fall 2012 Spools Results}

Table 8 below shows (a) the pre- and post-DCI results of the rear and front automobile question, (b) the online quiz results from the day before the Spool IBLA, and (c) the results from the final exam question (Figure 10).

You pull gently on the spool with a force $T$ and cause it to roll without slip. Which way will the friction force act?
a. To the left
b. To the right
c. Upwards
d. Not enough information to tell

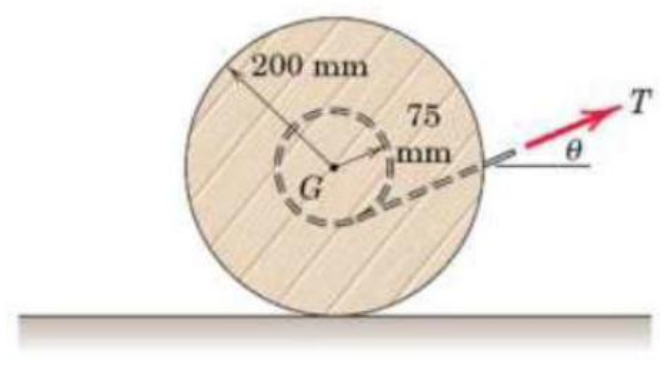

Figure 10. Final exam question assessing the Spool IBLA.

As a reminder, the DCI question is an indirect assessment of the Spool concept. The final exam question asked students to find the friction force acting on a spool when the rope is pulled at an angle, thus testing transfer of knowledge.

Table 8. Assessment of Spool IBLA in fall '12; Percentage of students answering the question correctly.

\begin{tabular}{|c|c|c|c|c|c|c|c|c|}
\hline \multicolumn{2}{|c|}{ DCI (Figure 9) } & \multicolumn{2}{|c|}{ DCI (Figure 9) } & \multicolumn{3}{c|}{ Online Quiz Problems (pre-IBLA) } & Exam \\
\hline \multicolumn{2}{|c|}{ Friction on Rear } & \multicolumn{2}{|c|}{ Friction on Front } & \multicolumn{2}{c|}{ Horizontal Pull } & \multicolumn{2}{c|}{ Vertical Pull } & Angle pull \\
\hline Pre & Post & Pre & Post & motion & friction & Motion & friction & \\
\hline $29.0 \%$ & $57.4 \%$ & $29.0 \%$ & $51.1 \%$ & $37.6 \%$ & $69.5 \%$ & $78.4 \%$ & $70.5 \%$ & $65.9 \%$ \\
\hline
\end{tabular}


The scores demonstrate conceptual improvement (around 25\% increases) from pre- to post- DCI assessments. The post-DCI score is considered low by the research team. Students exhibited beneficial understanding on the pre-IBLA quiz (around 70\%) and post-IBLA exam (66\%).

\section{Winter 2013 Results}

The following table records some of the student scores on the pre- and post -DCI, and postactivity quiz for the winter class. The DCI question is the same as that introduced earlier, with the automobile tires. The post-quiz featured a) one quantitative problem with a spool with a larger outer radius than inner radius, and b) a qualitative problem involving a spool subjected to a rope pulling vertically downwards (Figure 11). These problems permitted students to transfer the rolling concepts to a new context, and put their understanding to the test.

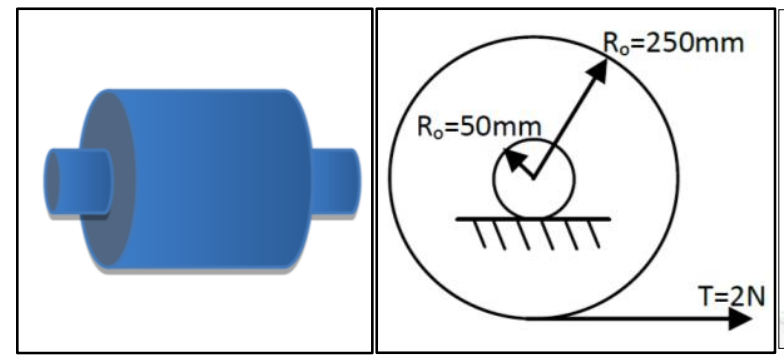

Figure 11. Diagrams from Quiz in winter 13. Part a) on Left.

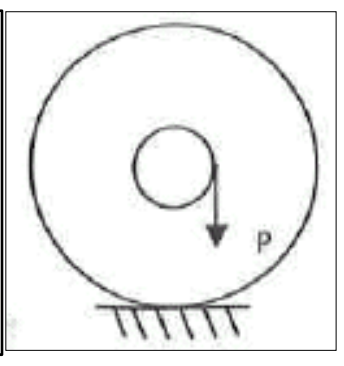

Part b) on right

Table 9. Assessment of Spool IBLA Winter 2013; Percentage of students answering the question correctly.

\begin{tabular}{|c|c|c|c|c|c|c|}
\hline \multicolumn{2}{|c|}{ DCI (Figure 9) } & \multicolumn{2}{|c|}{ DCI (Figure 9) } & \multicolumn{2}{c|}{ Post Activity Quiz } \\
\hline \multicolumn{2}{|c|}{ Friction on Rear } & \multicolumn{2}{|c|}{ Friction on Front } & $\begin{array}{c}\text { Q1: quantitative } \\
\text { inverse spool }\end{array}$ & $\begin{array}{c}\text { Q2: qualitative spool, } \\
\text { vertical pull down }\end{array}$ \\
\hline Pre & Post & Pre & Post & & $\begin{array}{c}\text { motion } \\
\text { direction }\end{array}$ & $\begin{array}{c}\text { friction } \\
\text { direction }\end{array}$ \\
\hline $37 \%$ & $42.9 \%$ & $33.3 \%$ & $35.7 \%$ & $76.3 \%$ & $69 \%$ & $82.7 \%$ \\
\hline
\end{tabular}

The learning gains from pre- to post-DCI question were small, less than ten percentage points. This demonstrates students did not really understand these concepts and this must be improved. Students exhibited decent understanding on the post-activity quiz. For example, students scored a 
mean of $76 \%$ on the first quantitative transfer question, while $69 \%$ of students correctly answered the question relating to which direction the spool will move, and $83 \%$ of students correctly determined the friction force direction.

Before running each hands-on activity, students made predictions as per the IBLA process. Students used their cell phones and Poll Everywhere to make individual predictions, and made a secondary prediction after conversing with teammates. These results are shown for the two scenarios in the following table.

Table 10. Correct Prediction Results, Winter '13.

\begin{tabular}{|l|c|c|}
\hline & Individual Predictions & Team Predictions \\
\hline $\begin{array}{l}\text { Horizontal pull - } \\
\text { motion direction }\end{array}$ & $26 \%$ & $35.7 \%$ \\
\hline $\begin{array}{l}\text { Horizontal pull - } \\
\text { friction direction }\end{array}$ & $56 \%$ & $75 \%$ \\
\hline $\begin{array}{l}\text { Vertical pull - } \\
\text { motion direction }\end{array}$ & $91 \%$ & $92.9 \%$ \\
\hline $\begin{array}{l}\text { Vertical pull - } \\
\text { friction direction }\end{array}$ & $32 \%$ & $42.9 \%$ \\
\hline
\end{tabular}

The prediction scores went up for all cases after talking amongst teammates — most evident in the horizontal-friction case ( $24 \%$ change). The vertical-motion case showed the most confidence, with little change from individual to team prediction (around $92 \%$ correct). The other items in the table show slight improvement as a result of team interaction.

The research team observed that students still had trouble grasping the concepts of friction force direction, and the spool motion direction. As a result, the professor in the winter '13 class utilized an intervention half-way through the activity, similar to the pulley IBLA. After each case (horizontal or vertical), the professor intervened the activity to discuss his logic with the class and show appropriate dynamic diagrams. He held a discussion about the friction direction and rolling direction, and called on students to explain their reasoning aloud. The professor also asked about 
the spool's magic angle, where the spool slides in place when the string is pulled at a certain angle. Then he asked if friction is tied to rolling direction (which it is not). This intervention could have cleared up confusion and promoted understanding of students. Thus students continued into the second case (vertical) with increased understanding after the intervention, and were able to 'mirror' the professor's logic path.

The class exhibited their thinking on the worksheets with correct conclusions. The majority of groups recorded the correct behavior of the plastic spool when writing down conclusions, such that the spools rolls left or right under the rope pull, and the friction acts either left or right. In addition, most students correctly stated the static friction force is less than or equal to $\mu_{\mathrm{s}} \mathrm{N}$, until slip occurs at which time fiction $=\mu_{\mathrm{K}} \cdot \mathrm{N}$.

On the other hand, students mentioned misconceptions, such as the friction force must always exist as an opposite to the pulling, tension force.

\section{Additional winter 2013 Results for Intermediate Class}

The activity was also implemented for an intermediate dynamics course with the following results.

Table 11. Results from Intermediate dynamics, Winter '13; correct responses

\begin{tabular}{|l|c|l|c|}
\hline \multicolumn{2}{|c|}{ Pre-activity Quiz } & \multicolumn{2}{c|}{ Team Predictions } \\
\hline $\begin{array}{l}\text { Horizontal pull - } \\
\text { motion direction }\end{array}$ & $52 \%$ & $\begin{array}{l}\text { Horizontal pull - } \\
\text { motion direction }\end{array}$ & $73 \%$ \\
\hline $\begin{array}{l}\text { Horizontal pull - } \\
\text { friction direction }\end{array}$ & $66 \%$ & & $84 \%$ \\
\hline $\begin{array}{l}\text { Vertical pull - } \\
\text { motion direction }\end{array}$ & $86 \%$ & $\begin{array}{l}\text { Vertical pull - } \\
\text { motion direction }\end{array}$ & \\
\hline $\begin{array}{l}\text { Vertical pull - } \\
\text { friction direction }\end{array}$ & $45 \%$ & $\mathrm{n}=65$ & \\
\hline & & & $\mathrm{n}=63$ \\
\hline
\end{tabular}


The prediction scores for the quiz and team interaction were fairly high, which should be expected because this class should have already learned the Spool concepts from the previous course. These predictions were on average greater than the beginner dynamics class predictions. The DCI assessment was again given after the activity, which can be seen in the following table. The final exam problem asks to find the rolling direction of a spool with the tension applied at an angle $\Theta$, which acts to generalize the problem.

Table 12. Percent Correct Scores from Spools in Intermediate dynamics, Winter'13

\begin{tabular}{|c|c|c|}
\hline DCI Post (Figure 9) & DCI Post $(\mathrm{n}=59)$ & Final \\
\hline Friction on rear tire & Friction on front tire & \\
\hline $55.9 \%$ & $47.5 \%$ & $64.3 \%$ \\
\hline
\end{tabular}

The post-DCI scores demonstrate decent understanding of friction direction concepts. Most students did not fully complete the worksheets due to a lack of class time. This class also featured a professor-led discussion which brought up relevant ideas and allowed students to check their explanations with the class.

\section{Spring 2013 results}

The activity was revised for the next implementation in spring by changing the wording and reorganizing the worksheet. Similar to the previous implementations, assessments were taken and the instructor guided the class discussion. In this session, the post-activity quiz asked students to find a) the acceleration of the spool (linear and angular) and b) the minimum coefficient of static friction compatible with the motion, with results shown below. 
Table 13. Percent Correct scores from Spools in Spring '13, Engineering Dynamics class

\begin{tabular}{|c|c|c|c|c|}
\hline \multicolumn{2}{|c|}{ DCI } & \multicolumn{2}{c|}{ DCI } & Post Activity Quiz \\
\cline { 1 - 3 } \multicolumn{2}{|c|}{ Friction on rear tire } & \multicolumn{2}{|c|}{ Friction on front tire } & \multirow{2}{*}{$\begin{array}{c}\text { Spool Question, a) and b })^{5} \\
\text { Combined score }\end{array}$} \\
\hline Pre & Post & Pre & Post & \\
\hline $44.4 \%$ & $59.4 \%$ & $29.6 \%$ & $40.6 \%$ & $77.4 \%$ \\
\hline
\end{tabular}

These DCI scores indicate moderate improvement from pre- to post- conditions, around fifteen percent change. The post-activity scores show an ability to solve the transfer problem and demonstrate understanding of the rolling concepts. The final exam percentage shows a decent number of students making correct transfer.

Teams again made predictions about the resultant spool motion and the friction direction, for the horizontal and vertical pull cases. Individual predictions were done with Poll-Everywhere website with cell phones (which had few responses due to setup error). Team predictions were recorded on the worksheet. Both items are shown in the following table.

Table 14. Percent Correct Spool predictions for Spring '13

\begin{tabular}{|l|c|c|}
\hline & $\begin{array}{c}\text { Individual Predictions } \\
\mathrm{n}=\sim 15\end{array}$ & $\begin{array}{c}\text { Team Predictions } \\
\mathrm{n}=30\end{array}$ \\
\hline $\begin{array}{l}\text { Horizontal pull }- \\
\text { motion direction }\end{array}$ & $9 \%$ & $20 \%$ \\
\hline $\begin{array}{l}\text { Horizontal pull }- \\
\text { friction direction }\end{array}$ & $44 \%$ & $46.7 \%$ \\
\hline $\begin{array}{l}\text { Vertical pull - } \\
\text { motion direction }\end{array}$ & $59 \%$ & $76.7 \%$ \\
\hline $\begin{array}{l}\text { Vertical pull - } \\
\text { friction direction }\end{array}$ & $72 \%$ & $60 \%$ \\
\hline
\end{tabular}

${ }^{5}$ a) the angular acceleration of the disk and the linear acceleration of $G$.

$b$ ) the minimum value of the coefficient of static friction compatible with this motion. 
Students did not predict the horizontal pull motion and friction questions very well (less than one quarter of the class was correct). The vertical pull predictions were slightly better than the horizontal scenario.

Unfortunately, some student groups wrote on their worksheets the wrong friction direction even after running the physical experiment (and thus keeping with their prediction). Students may have made this mistake because the friction direction is not directly observable. Fortunately, the same groups wrote the correct spool motion direction on the worksheet, which should be obvious from viewing the experiment.

Teaching assistants facilitated this activity because the professor was unavailable. After each scenario the assistants spoke to the class to explain the correct behavior and dynamic principles. Unfortunately, students spent more time than needed, getting hung up on some details (deciding if the spool was supposed to roll without slip or slide when pulling on the string).

\section{Additional Combined Results}

Later in the quarter students filled out a survey questionnaire which asked: When did the behavior of the spool finally make sense to you (e.g. in the middle of the activity, after you talked to your team about it, after it was discussed in class, when you took the quiz, after you saw the quiz solution, after you did the homework problem, after you saw the homework solution, it still doesn't make sense....)? See the table below for a count of their responses. 
Table 15. When did the spool concepts make sense to you? Engineering Dynamics class

\begin{tabular}{|l|c|c|c|}
\hline & Fall '12 & Winter '12 & Spring '13 \\
\hline Understood beforehand & $6 \%$ & $0 \%$ & $0 \%$ \\
\hline During or after Pre-quiz & $6 \%$ & N/A & N/A \\
\hline During activity & $\mathbf{2 1 \%}$ & $\mathbf{2 4 \%}$ & $10 \%$ \\
\hline Talking with team & $\mathbf{2 5 \%}$ & $8 \%$ & $13 \%$ \\
\hline After activity & $4 \%$ & $0 \%$ & $3 \%$ \\
\hline Discussion in class & $\mathbf{2 2 \%}$ & $\mathbf{4 0 \%}$ & $\mathbf{3 8 \%}$ \\
\hline Studying it later & $3 \%$ & $4 \%$ & $10 \%$ \\
\hline During or after Post-Quiz & N/A & $12 \%$ & $10 \%$ \\
\hline After homework & $0 \%$ & $8 \%$ & $10 \%$ \\
\hline Still confused & $13 \%$ & $4 \%$ & $10 \%$ \\
\hline Total class size & $n=168$ & $n=25$ & $n=31$ \\
\hline
\end{tabular}

Bold entries in the table are significant because they represent the most densely populated times when understanding occurred, as reported by the students. For instance, the significant times of understanding occurred during the activity, while talking with teammates, and during the class discussion. The instructor should take advantage of the learning gains during these three time points and focus teaching efforts. In addition, instructors can improve the teaching during the minor time points where less learning took place.

In fall we did not do an intervention; in winter and spring there was an intervention. With intervention there was more discussion with the class and instructor. As stated in the survey, fewer students were confused when the intervention was held, $4 \%$, vs. $13 \%$ without intervention (percentage from 'still confused' from the table).

Students filled out a survey after the activity date, with questions and responses found below. The first two questions used a Likert scale (ranging from $1=$ strongly disagree, up to $5=$ strongly agree), while the third question used a ranking from one to eleven $(1=$ most important down to $11=$ least important). This can be seen in the following table. 
Table 16. Survey Results

\begin{tabular}{|c|c|c|c|}
\hline $\begin{array}{c}\text { Pulling the spools } \\
\text { helped me learn } \\
\text { dynamics. } \\
\text { (Likert scale) }\end{array}$ & $\begin{array}{c}\text { Pulling the spools } \\
\text { was interesting } \\
\text { and motivating. } \\
\text { (Likert scale) }\end{array}$ & $\begin{array}{c}\text { Importance of } \\
\text { Activity relative } \\
\text { to other activities. } \\
(1 \text { is most, } 11 \text { is } \\
\text { least) }\end{array}$ & Class session \\
\hline $4.18 / 5$ & $3.81 / 5$ & $7.12 / 11$ & Fall '12 \\
\hline $4.27 / 5$ & $3.92 / 5$ & $6.31 / 10$ & Winter '12 \\
\hline $3.6 / 5$ & $3.3 / 5$ & $6.7 / 11$ & Spring '13 \\
\hline
\end{tabular}

Students reported the Spool activity to be helpful and motivating to learn dynamics concepts. The activity was ranked lower on the list of importance relative to other class activities (which included lecture, PowerPoint examples, reading the textbook, rolling objects activity, other projects, homework, team quizzes, and class discussion). On average from the fall, winter, and spring, students responded that the most important activity to learn course material was lecture.

\section{Spool Discussion}

The friction direction and rolling direction of the spools are not obvious initially, but later discovered when dynamic analysis is carried out. The prediction scores were low and varied from course to course. Most of the students from the dynamics class were successful in figuring out the friction direction and motion direction in response to a pull force at different angles and locations. A big influence in their understanding may be the professor intervention after both the horizontal pull case and vertical pull case, in which the professor answered questions and gave his explanation. Student commented in the survey that this discussion in class helped to clear up the concepts. Therefore the instructor played a major component in guiding the correct concepts of this inquiry activity.

The behavior of the spool under applied rope pulling was non-intuitive to some students. One might think that spools roll in the opposite direction of pulling, much like a toilet paper roll rolling away. But these spools displayed the opposite effect of rolling towards the direction of 
pulling, when the rope is wound underneath the inner column. Another difficulty is that you cannot see the friction force. The professor stated that students perform more poorly on the spool activity than simpler activities because the spool topic has more than one concept going on at once (friction force and applied load not at the mass center). The activity hits concepts from multiple angles (horizontal pull, vertical pull, angle pull). This forces them to apply concepts through multiple cases.

The DCI scores measured the level of understanding at the beginning and end of the course. The DCI scores show mild to medium levels of conceptual improvement. The transfer questions on the quiz and final exam tested the students to analyze the rolling motion of a new problem context. The quiz and final scores indicate medium levels of understanding (scores range from $65-80 \%$ correct) and indicate improvement from the beginning of the course.

In conclusion, once students overcame their apprehension of the spool rolling behavior from performing the activity, they were able to make sense of the concepts of rigid body rolling without slip. The activity is not as successful as the mass-pulley activity because it is more challenging (contains sum of forces and moments) and the friction force is not directly observable. This activity will continue in future classes and improve upon previous implementations.

\section{Spools Improvements}

The spool activity has some room for improvement in its implementation. One problem is that some students were confused about how hard to pull the string. The instruction team told them to pull softly. Like the other inquiry activities, the instructors do not first perform the experiment to instruct the class. The professor must be clear in guiding the inquiry activity to avoid student confusion. 
There is a need to somehow visually represent the friction force between the spool and the ground. Such a need is being addressed with a computer simulation showing the spool rolling behavior and friction presence. There is a question as to whether simulation is better than experiment; including advantages such as low cost and low resource use, but at the expense of perhaps less credibility or belief by the students. Feedback from students about the simulations will dictate if it is a beneficial addition to the activity. 


\section{4: ROLLING OBJECTS IBLA}

Students investigated the principles of mass

moment-of-inertia and work-energy in the

rolling objects IBLA. Students were evaluated

with a Dynamics Concept Inventory (DCI), a

pre-quiz, hands-on activity, survey, and a final

exam question, which were analyzed by the

professor and teaching assistants to gain insight

into student thinking and to improve course

outcomes. Two implementations will be

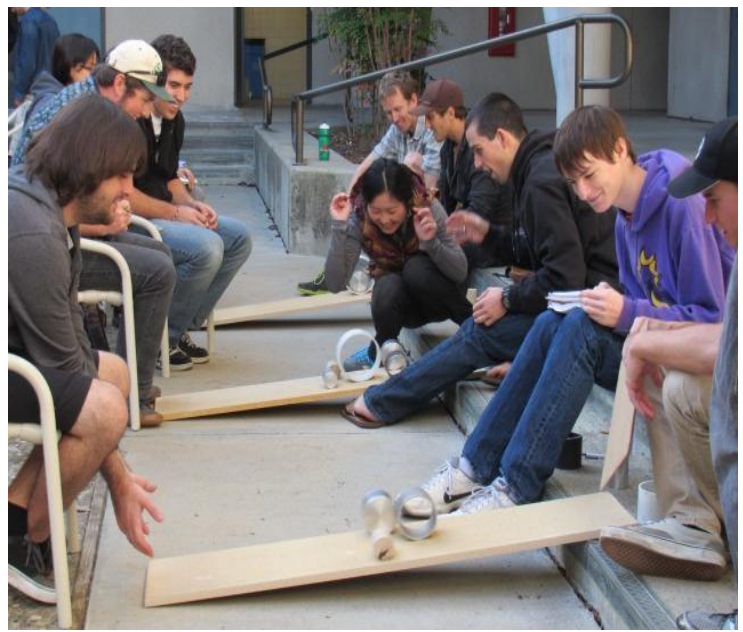

Figure 12. Rolling Objects IBLA

discussed: (a) a full IBLA where teams of 4-5 students manipulate the different objects, and (b) a demonstration mode in front of a class of 60 students.

\section{Implementing the Rolling Objects IBLA}

Students released objects to roll down an inclined ramp and observed how the different objects reached the bottom with different speeds (Figure 12). Objects ranged from metal pipes to wooden solid cylinders to plastic pipes, with varying radii and masses.

Students proceeded through the activity by making a prediction before each test case, then running the experiment. Next, they recorded the results on a worksheet, and explained their conceptual understanding. Through the inquiry activity, students confronted their predictions and previous ideas, and later went on to create informed conclusions. For example, a student initially thought an object rolls fast because it is lightweight, but later went on to learn the full description of why it behaves using dynamics principles, including the effects of varying mass and radius.

During the lab experience, the professor and teaching assistants oversaw the activity alongside the undergraduate students. They were able to aid the students, ask them thought-provoking questions, 
and guide them towards the correct conceptual understanding. For example, if the students roll a given set of objects and had inconsistent results, the assistants would have them repeat the roll a few more times to make sure the correct conclusion was reached.

Work-energy and mass moment of inertia are important principles in understanding rotating dynamics, and are assessed on the DCI. For this reason these concepts are the main focus of this IBLA. Additional concepts include: a) the mass moment of inertia is based upon mass distribution, and objects with the same 'form' of moment of inertia behave similarly when rolling, and b) potential energy of the rolling object at the top of the ramp is transferred into both rotational and translational kinetic energy at the bottom of the ramp.

The experiment compares solid cylinders and pipes as they roll without slip. Objects that are homogenous solids are called 'solid cylinders', while objects with hollow insides are referred to as 'pipes' (Figure 13). Solid cylinders roll differently than pipes due to different mass distributions about the rolling axis, referred to as its moment of inertia. The full dynamics analysis will be explored further later.

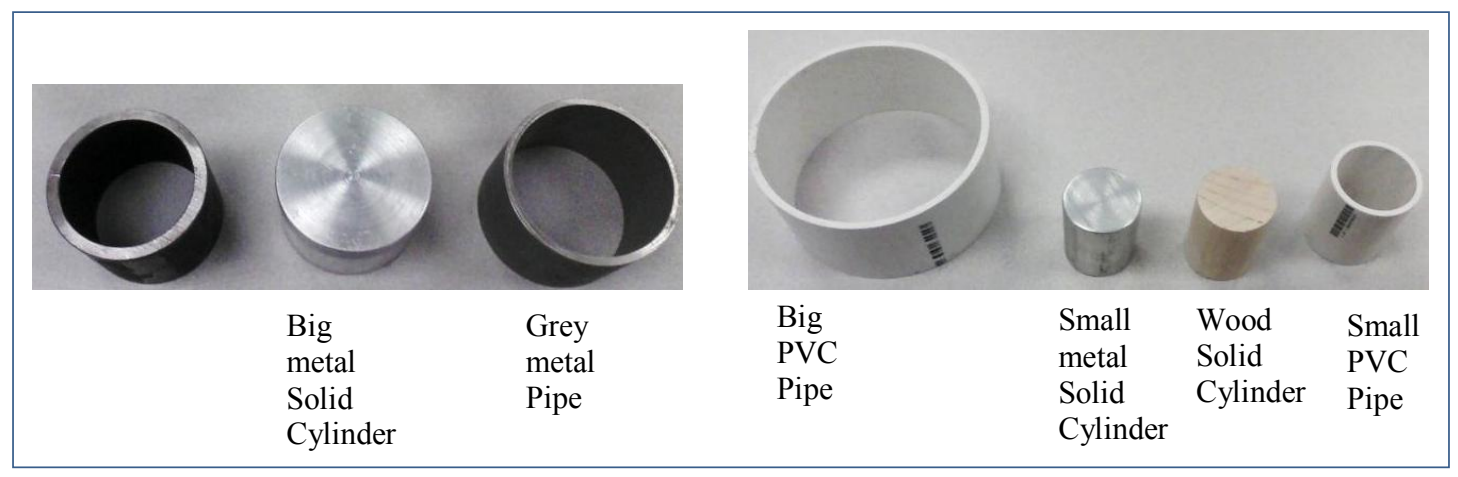

Figure 13. Collection of items for the Rolling IBLA

The Rolling Objects activity addresses the effects of distribution of mass with the first exercise (big metal solid cylinder and the black metal pipe with same radius, length, and mass). The IBLA then goes on to explore different concepts of work and energy by comparing races between different 
combinations of solids and pipes with varying radii and masses. By testing different combinations of objects, common student misconceptions are challenged using multiple scenarios.

This demonstrates to students that as long as there is rolling without slip all solid homogeneous cylinders will have the same linear velocity at the end of the ramp, independent of mass and radius. Furthermore, all cylinders will always get to the bottom of the ramp before all pipes, regardless of their radius and mass. This is surprising to students. To gain a more detailed understanding, one can look at the dynamics equations as follows.

This is demonstrated by examining the work-energy equation: $T_{1}+V_{1}=T_{2}+V_{2}$, where $T$ and $V$ are kinetic and potential energy, respectively. If the cylinder starts from rest, then $T_{1}=0$. For a given ramp, the change in height will be the same for all circular objects. Therefore, we can rewrite the equation as:

$$
m g h=\frac{1}{2} I_{G} \omega^{2}+\frac{1}{2} m v_{G}^{2}
$$

We now set the mass moment of inertia equal to $I_{G}=c m r^{2}$, where $c$ is a scaling factor. For a thin ring, $c=1$, and for a solid cylinder, $c=1 / 2$. If we also substitute the roll without slip condition, $\omega=v_{G} / r$, we obtain:

$$
m g h=\frac{1}{2} c m r^{2}\left(\frac{v_{G}^{2}}{r^{2}}\right)+\frac{1}{2} m v_{G}^{2}
$$

Solving for $v_{G}$, we see that the mass and the radius both cancel.

$$
v_{G}=\sqrt{\frac{2 g h}{1+c}}
$$


Examining Eq (3) shows that the linear velocity $v$ only depends on the mass moment of inertia factor, $c$. Therefore a round object with a higher mass moment of inertia coefficient 'c' will get to the bottom of the ramp more slowly than an object with a smaller ' $c$ '. Many students realized that this really indicates a distribution of the translational and rotational kinetic energy of the objects. A cylinder will have greater translational energy than a pipe of identical radius and mass; the pipe will have a greater rotational kinetic energy due to its greater mass moment of inertia. When released from identical locations on the ramp, the solid will reach the bottom fastest due to its greater translational speed.

\section{Rolling Objects Results}

To assess changes in conceptual understanding, the research team offered multiple assessment items.

Students were assessed with a DCI question (pre- and post-), a pre-activity-quiz, and a prediction sheet. During the activity, student teams completed a worksheet. Two-three weeks later, students completed a post-activity exam question and subjective survey (see later sections for more information). The results are shown in the following table and discussed later.

Table 17. (a) The pre- and post-DCI results of the rolling objects question along with normalized gain, (b) the quiz results from the day before the IBLA, and (c) exam results from after the IBLA.

\begin{tabular}{|c|c|c|c|c|c|c|c|}
\hline \multirow{2}{*}{ Class } & \multirow{2}{*}{$\begin{array}{l}\text { Number } \\
\text { of } \\
\text { Students }\end{array}$} & \multirow{2}{*}{$\begin{array}{c}\text { Activity } \\
\text { Type }\end{array}$} & \multicolumn{3}{|c|}{ DCI } & \multirow{2}{*}{$\begin{array}{c}\text { Quiz } \\
\text { (pre-IBLA) }\end{array}$} & \multirow{2}{*}{$\begin{array}{c}\text { Exam } \\
(\text { post-IBLA) }\end{array}$} \\
\hline & & & Pre & Post & $\begin{array}{l}\text { Normalized } \\
\text { gain }\end{array}$ & & \\
\hline Fall'12 & 169 & IBLA $^{6}$ & $31.3 \%$ & $89.8 \%$ & 0.852 & $43.4 \%$ & $84.5 \%$ Exam \\
\hline Fall'12 & 60 & Demo $^{7}$ & $58.1 \%$ & $55.7 \%$ & * 0.0413 & & \\
\hline Winter ' 13 & 29 & IBLA $^{6}$ & $25.9 \%$ & $89.3 \%$ & 0.856 & $20.7 \%$ & $\begin{array}{c}57.7 \% \\
\text { Ranking Task }\end{array}$ \\
\hline $\begin{array}{l}\text { Spring'13 } \\
\text { (Interm.) }\end{array}$ & 70 & IBLA $^{6}$ & $38.8 \%$ & $88.7 \%$ & 0.815 & & $69 \%$ Midterm \\
\hline Spring'13 & 34 & IBLA $^{6}$ & $14.8 \%$ & $93.8 \%$ & 0.927 & $64.7 \%$ & \\
\hline
\end{tabular}

$*$ Different normalized gain formula used for unusual case of Pre $>$ Post.

$\mathrm{G}=($ Post- Pre) / Pre

${ }^{6}$ Data from Cal Poly SLO

${ }^{7}$ Data from Eric Wang at University of Nevada, Reno 


\section{Implementing the Rolling Objects Demonstration}

At the University of Nevada, Reno (UNR) dynamics is a semester-long course taught in a traditional large, lecture style format (90-100 students is typical). In an effort to repair misconceptions concerning inertia, an in-class demonstration is conducted that lasts one full lecture period (50 minutes).

Personal response devices (a.k.a. "clickers") are used daily to enhance student involvement. For this study, the clicker responses were used in lieu of a pre-test. It must be noted that students are allowed to discuss the question posed before answering, which confounds the results.

When prompted (via a PowerPoint slide) whether an aluminum or steel solid cylinder would have a higher speed at the bottom of a ramp, $37.4 \%$ of students indicated steel, $40.7 \%$ indicated aluminum, and $22.2 \%$ indicated they would have the same speed (correct).

Likewise, when asked whether an aluminum cylinder or aluminum hoop would have a higher speed at the bottom of a ramp, $58.1 \%$ students chose the cylinder (correct), $31.1 \%$ chose the hoop and, $10.8 \%$ indicated they would have the same speed. This pretest implied students have misconceptions about the behavior of rolling objects.

After the initial questions were posed, the rest of the class period was devoted to demonstrating how different objects behaved as they rolled down a ramp. The equations discussed above were also covered followed by more demonstrations using cylinders and hoops with varying mass, radii and inertia.

\section{Additional IBLA Results}

Students predicted the rolling behavior of the objects before each test case. Such predictions are tabulated below for each class. The questions ask for the race result between different pipe and solids. The predictions questions are as follows. Note: the question number in Table 19 corresponds to the scenario shown in Table 18. 
Table 18. Prediction Questions shown as pictures

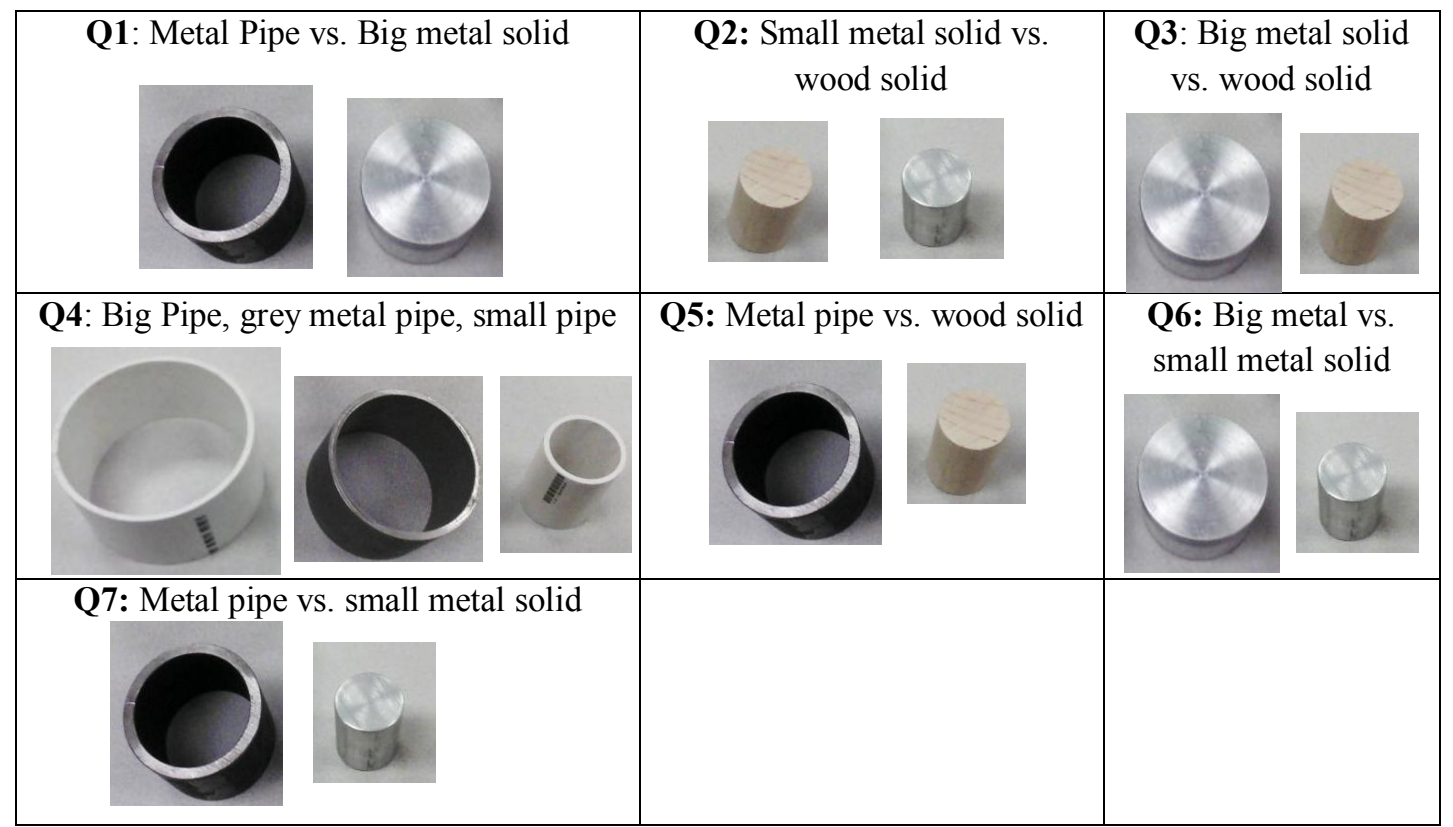

Table 19. Predictions for object races, by class and question \# (see Table 18). (intm) $=$ Intermediate Dynamics course

\begin{tabular}{|c|c|c|c|c|}
\hline Fall '12 & Winter'13 & Spring'13 (intm) & Spring '13 (intm) & Spring'13 \\
\hline $\begin{array}{c}\text { from worksheet } \\
(\mathrm{n} \sim 160)\end{array}$ & $\begin{array}{c}\text { from worksheet } \\
(\mathrm{n}=29)\end{array}$ & $\begin{array}{c}\text { from Poll- } \\
\text { everywhere } \\
(\mathrm{n}=51)\end{array}$ & $\begin{array}{c}\text { from anonymous } \\
\text { worksheet } \\
(\mathrm{n}=58)\end{array}$ & $\begin{array}{c}\text { from } \\
\text { anonymous } \\
\text { worksheet } \\
(\mathrm{n}=23)\end{array}$ \\
\hline Q1: $81 \%$ & Q1:51.7\% & Q1:56.8\% & Q1: 72.4\% & Q1: $95.6 \%$ \\
Q2: $25 \%$ & Q2: $17.2 \%$ & & Q2: $31.0 \%$ & Q3: $31.0 \%$ \\
Q3: $16 \%$ & Q3: $20.7 \%$ & & Q4: $48.3 \%$ & Q4: $56.5 \%$ \\
Q4: $63 \%$ & Q4: $24.1 \%$ & & Q5: $74.1 \%$ & Q6: $13 \%$ \\
& & & & Q7: $87 \%$ \\
\hline
\end{tabular}

From the results table, students predicted the first question reasonably well (over 50\%). Some of them have seen this question in previous physics classes. In question two, most students thought the objects would not tie because one is heavier than the other. In question three, students thought the wood object would roll faster because it is lighter than the big aluminum solid. Question four was predicted well, after students began to develop the correct concept that objects with the same form of moment of inertia roll the same. Question five was predicted well by the intermediate 
class. The sixth question was scored poorly, where students thought the small solid would beat the big solid. Students still hold misconceptions at this stage. The seventh question was predicted well, students seem to understand the concepts by this last prediction stage.

The prediction sheets were reviewed to look at conceptions, misconceptions, and themes from Fall to Spring classes. In Fall'12: The concepts from the team prediction sheet included recognizing that the pipe has a bigger moment of inertia than the solid, and that it has mass concentrated farther away from its rotational axis; mass/radius cancel from the work-energy equation; and bigger rotational velocity ' $\omega$ ' because of smaller radius ' $r$ '. On the other hand, misconceptions were revealed such as: $\mathrm{I}_{\text {pipe }}<\mathrm{I}_{\text {solid }}$ (incorrect) and that the work-energy equation was missing terms. Some teams exhibited an improved progression through the worksheet and improved dynamics understanding.

As teams progressed through the different stages of the activity, their conceptual understanding began to match dynamics principles; they used the W.E equation to show why race results occur, and refined previously held beliefs (eg. incorrectly thought wood solids rolls faster than metal solids). From performing each case, students learned inductively. They built the general idea that rolling behavior is based on the form of 'I', and that solids beat hoops. Each group had its own reasoning for explaining things.

In the winter' 13 class there were no explanations on prediction sheet so the pre-activity quiz was investigated. In a pre-activity quiz, the race between big solid vs. big pipe was asked. Students correctly used the work-energy equation, canceled mass, and recognized that the 'I' affects the rolling acceleration. Some misconceptions were stated that the net force was the same so the acceleration should be equal, and stating that mass located far from the axis of rotation caused greater acceleration. 
In the Spring'13 class there were no explanations on the prediction sheet, only tally marks. On the pre-quiz, Students ( 22 out of 34 ) correctly reasoned that the cylinder reaches the bottom faster due to lower moment of inertia, but few people put the pieces together to cancel mass and radius. The activity can help to realize this objective. Students ( 6 out of 34 ) wrote out the work-energy equation. Some people made a good connection to the previous figure skater example — holding arms out produces a different moment of inertia than when arms are pulled in. Some students wrote down starting points of analysis but stopped short of reaching the final conclusion, then made a semi-supported guess to answer the prompt. And out of the people who chose the wrong answer B, most of them showed proper logic albeit missed the correct form of the moment of inertia. The activity can help to advance conceptual understanding.

In Spring'13 intermediate class, anonymous prediction sheets were assessed. Students did well on the first question (big solid vs. big pipe) - this question had been seen before in poll everywhere. Then predictions were below 50\% for Q2-Q4. Finally, students did well on the last prediction Q5 (wood solid beats big metal pipe). Perhaps the IBLA taught them something so by the end they were able to make accurate predictions.

Students were tested on Dynamics concepts on an activity worksheet, as can be seen in Table 20; worksheet responses were broken up into themes in the left hand column. The right column lists the percentage of students who reported the concept or statement. This allows the investigation of which aspects of the material are getting through to the students, providing more specific feedback to the instructor. 
Table 20. Categorizing student in-class worksheet responses Fall'12 IBLA

\begin{tabular}{|l|c|}
\hline $\begin{array}{l}\text { Concept or Statement written explicitly } \\
\text { on worksheet }\end{array}$ & $\begin{array}{l}\text { Percent of teams } \\
\text { responding }\end{array}$ \\
\hline Moment of Inertia based upon mass distribution & $38.8 \%$ \\
\hline $\begin{array}{l}\text { Moment of Inertia relates to rolling acceleration or } \\
\text { translating velocity }\end{array}$ & $67.4 \%$ \\
\hline $\begin{array}{l}\text { Potential Energy at top of ramp converts to Kinetic } \\
\text { Energy at the bottom of ramp }\end{array}$ & $75.5 \%$ \\
\hline $\begin{array}{l}\text { Kinetic energy distributes into linear and angular } \\
\text { components }\end{array}$ & $44.9 \%$ \\
\hline Work-Energy equation & $59.2 \%$ \\
\hline Solid cylinders beat hoops down ramp & $2.1 \%$ \\
\hline $\begin{array}{l}\text { Solid cylinders roll the same down the ramp; } \\
\text { pipes roll the same down the ramp }\end{array}$ & $22.5 \%$ \\
\hline
\end{tabular}

The most stated concept was the 'conversion from potential to kinetic energy' (75.5\%); while the least stated concept was that 'solid cylinders beat hoops down the ramp' $(2.1 \%)$. Some of these concepts were addressed in subsequent quarters in hopes of building attention to these concepts. A question was specifically added to the worksheet that 'all solids roll the same' and another question asked whether 'all thin walled hoops rolled the same'.

The research team must take caution when making conclusions from students' responses. Perhaps only a minority of the group decided what to write down, and understanding could be deeper than what was written on the worksheets. Perhaps the format of the worksheet influenced learning outcomes. For example, some of the concepts were explicitly included in the question prompt, while others were not. The research team hoped that the students would think critically and reach the right conclusion for each prompt.

Students were again assessed by a midterm or final exam question, depending on the class. The midterm question for the Spring' 13 intermediate class is shown next. 
1) (10 pts) Each of the objects below is released from rest at the top of a ramp. Rank in order which object will get to the bottom of the ramp the fastest (assume rolling without slip).

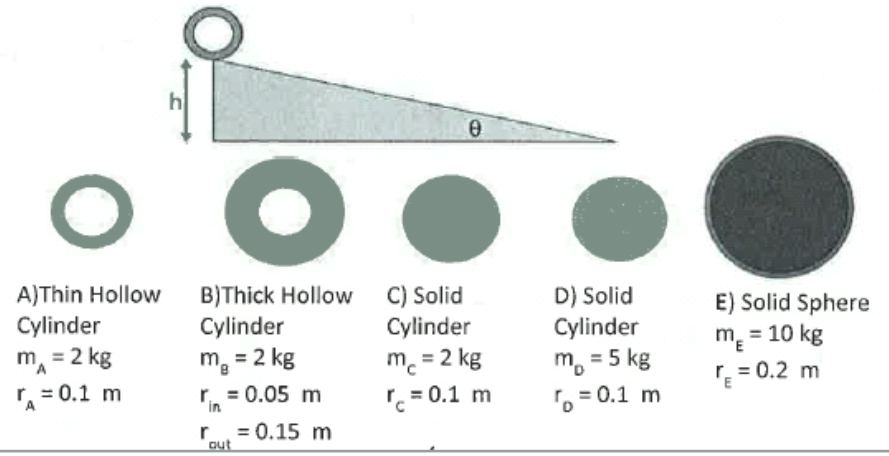

Figure 14. Midterm ranking question, Spring '13 intermediate.

These values can be seen in Table 17 and Table 21.

Table 21. Midterm question assessment, Spr '13 Intermediate class

\begin{tabular}{|c|c|c|}
\hline \multicolumn{2}{|c|}{ Concepts mentioned in explanation } & Score \\
\hline Speed is independent of mass and radius & $40.0 \%$ & Mean score: $6.9 / 10 \quad(n=70)$ \\
\hline Moment of inertia coefficient & $27.1 \%$ & 5 mint \\
\hline Distribution of mass & $25.7 \%$ & correct rank and 5 points given for \\
\hline $\begin{array}{l}\text { Rotation/kinetic components of kinetic } \\
\text { energy }\end{array}$ & $7.1 \%$ & correct conceptual explanation. \\
\hline
\end{tabular}

Students in the intermediate class recognized that mass and radius cancel from the work energy equation (40\%), which is a major conclusion to reach in the module of rolling objects. The remaining categories were presented less by students. One misconception listed was that speed is directly proportional to moment-of-inertia value, which is not always true. Note: Some concepts can be understood but may have not been explicitly stated by students.

\section{Rolling Objects Subjective Assessment}

Additionally, they were asked "When did the behavior of the different rolling cylinders finally make sense to you (e.g. in the middle of the activity, after talking to your team about it, after it 
was discussed in class, when you took the quiz, after you saw the quiz solution, it still doesn't make sense...)?" Responses were coded in Table 22, which helps the professor to pinpoint when the students experienced the 'aha' moment and understood the course concepts.

Table 22. Student responses as to when they understood the concepts in the IBLA. (Engineering Dynamics only, data collected from survey)

\begin{tabular}{|l|c|c|c|}
\hline \multirow{2}{*}{ When in time understood concepts } & \multicolumn{3}{|c|}{ Quantity of response } \\
\hline & Fal.'12 & Win.'13 & Spr.'13 \\
\hline Understood beforehand & $6 \%$ & - & $6 \%$ \\
\hline During/after pre-quiz & $1 \%$ & - & - \\
\hline During activity & $\mathbf{3 1 \%}$ & $\mathbf{4 2}$ & $\mathbf{2 5 \%}$ \\
\hline Talking with team & $\mathbf{2 2 \%}$ & $15 \%$ & $16 \%$ \\
\hline After activity & $4 \%$ & $4 \%$ & $3 \%$ \\
\hline Discussion in class & $11 \%$ & $8 \%$ & $16 \%$ \\
\hline Look at equations & - & - & $\mathbf{1 9 \%}$ \\
\hline Studying it later & $7 \%$ & - & - \\
\hline After homework & $\mathbf{1 3 \%}$ & $\mathbf{2 7 \%}$ & $12 \%$ \\
\hline After post-quiz & N/A & $4 \%$ & N/A \\
\hline Still confused & $4 \%$ & - & $3 \%$ \\
\hline (Total class size) & $(166)$ & $(26)$ & $(32)$ \\
\hline
\end{tabular}

Students were asked a number of questions on an end-of-course survey. They were able to express their opinions and rate course content. The first set of questions asked (a) if different course components helped the students learn the material and (b) if it was interesting and motivating (Likert scale: $1=$ strongly disagree, $2=$ disagree, $3=$ neutral, $4=$ agree, and $5=$ strongly agree). The next question asked students to rate how the IBLA helped you learn the material relative to other course material (rank from 1 to 11). Averages for the responses are shown in Table 23. 
Table 23. Results from end of the course survey (no intermediate class survey data)

\begin{tabular}{|c|c|c|c|}
\hline & $\begin{array}{c}\text { The Rolling } \\
\text { IBLA helped } \\
\text { me learn the } \\
\text { material }\end{array}$ & $\begin{array}{c}\text { The Rolling } \\
\text { IBLA was } \\
\text { interesting and } \\
\text { motivating }\end{array}$ & $\begin{array}{c}\text { Activity helped you learn the course } \\
\text { material relative to other course material. } \\
\text { (i.e. the number 1 ranking helped you the } \\
\text { most, 2 the second most, to 11 least) }\end{array}$ \\
\hline Fall'12 & $4.38 / 5$ & $4.12 / 5$ & 6.43 \\
\hline Win.'13 & 4.54 & 4.23 & 6.27 \\
\hline Spr.'13 & 4.16 & 3.75 & 6.28 \\
\hline
\end{tabular}

The values from the first two questions, at an average of $4 / 5$, demonstrate overall student satisfaction with the activity being helpful and interesting, and mildly helpful for learning course material relative to other course material.

Video footage was taken to witness student learning progress during the Fall activity. Through the recordings, the research team could investigate students' justifications and thought processes while they answered the different prompts. From the video footage, one group of students began to see a trend in the outcomes. For example, one student reported that "mass and radius did not affect rolling behavior." Furthermore, by the end of the worksheet they started to make the correct predictions, such that "all pipes would roll the same." One group compared the gravity force from a large cylinder to the large moment of inertia it possessed. One group mentioned, "Gravity force gets bigger with cylinder/pipe mass, but longer to accelerate." One group stated their "predictions were wrong", showing they were perceptive of their previously held misconceptions, which can later be repaired with the correct conclusions. Most groups managed to stay on task - usually one person in the group acted as the writer, while another acted as the lead "roller." 


\section{Misconceptions}

Some common misconceptions from this activity are as follows:

- "Speed is proportional to moment-of-inertia value magnitude." This semi-misconception is that rotational acceleration is based upon the magnitude of mass moment of inertia $(\vec{M}=$ $I \vec{\alpha})$, when the 'form' of MOI is what really matters. This concept can explain many of the rolling object cases, but is not entirely correct.

- "Mass is a determining factor, lighter objects are faster" or "heavier objects roll faster." Students may think that heavier objects roll more slowly, but one must look at the distribution of mass rather than the value of the mass, as well as the coefficient of the moment of inertia. Mass ends up canceling out of the work-energy equation, so it does not come into play.

- "The ratio of two objects' moments of inertia was equivalent to the ratio of their radius or the ratio of their mass". $I_{\text {metal }} /_{I_{\text {wood }}}=r_{\text {metal }} / r_{\text {wood }}=m_{\text {metal }} / m_{\text {wood }}$ (false). This misconception was to explain rolling behavior of two solid cylinders of different radius and mass (eg. some guessed that the wood solid would beat the metal solid, which is untrue). Such misconceptions sometimes correctly explain rolling behavior but do not hold in all cases.

- Some groups felt the time crunch and sought to finish the activity quickly and write something down on paper, including incomplete reasoning schemes, even if they were not fully sure of their results.

\section{Compare Demonstration to Small Group IBLA}

As can be seen in Table 17, the students who participated in the IBLA scored considerably higher on the DCI post question (Appendix) than those who witnessed the demonstration. Although this cannot be attributed totally to the IBLA, it does suggest that active participation in the activity and continued testing and discussion of different rolling objects may have a large effect on student understanding. The follow-up homework assignment may also play a large role in the outcome: asking students to make calculations after doing the physical activity could have strongly reinforced the IBLA. From Table 22, about $15 \%$ of students reported they had their 'aha' 
moment during the homework. A comparison of the IBLA and demonstration mode certainly bears additional investigation before the comparison can be more accurately defined.

\section{Discussion}

The first four implementations of the IBLA were largely successful. Student scores on a relevant DCI question were nearly three times higher at the end than at the beginning of the course, and $44 \%$ higher than a control group where a similar demonstration was provided. The IBLA forced students to make predictions, directly confront their misconceptions, and formulate new conceptual frameworks to explain the behavior of the rolling objects. It is hypothesized that the follow-on homework assignment helped to solidify this new conceptual framework and improved student understanding of mass moments of inertia and the principles of work-energy. Through the hands-on activity the majority of students reached correct conclusions from the rolling behavior of the objects. Students seemed to see the critical concepts: rolling behavior is independent of mass and radius, and depends on the distribution of mass and the coefficient of the moment of inertia.

Students brought misconceptions to the activity, which were revealed through their predictions. Students got hung up on the concepts of the correct form of moment of inertia (which can be looked up in a book), and not understanding all the terms of the Work-Energy equation.

One issue that arose was that a small difference in the starting position changed the final outcome, so that two solid cylinders may not reach the bottom of the ramp at exactly the same time (when they should tie). This issue was addressed by utilizing a proper starting gate. Interestingly, students will cling to their previously held misconceptions even if there is only a slight difference in velocities at the bottom (e.g. a steel cylinder just barely beats a wooden cylinder), which is interesting because students are shaping the experimental outcome to match their misconceptions. 
From the coded responses in Table 20, understanding of the lower percentage scoring areas (example: solid cylinders always beat hoops down ramp) could be improved through new methods or by more effort in current methods. Although moment-of-inertia is an important concept it was only shown by $38 \%$ in student's worksheet responses - there is room for improvement. Another concept, work-energy equation, an important dynamics relationship, was stated on $59.2 \%$ of group worksheets. Both of these topics were covered on the follow-up homework assignment. Emphasis could be added to promote such concepts and steer the student in the right direction towards the right answer. Improvements could be implemented by a question explicitly probing this idea or by more coaching to direct the student. Such questions would elicit students' held misconceptions, which can then be repaired.

From the survey represented in Table 22, performing the physical activity proved to be a significant influence in understanding of the subject as well as talking with teammates. Therefore the instructor should spend class time using the physical activity and letting students talk in teams. One teaching assistant noted that when students collaborated with others, they did well.

Survey comments show that student understanding grew because of the activity, and found the activity motivating and helpful to their learning.

\section{Rolling Objects Improvements}

Though this activity is successful in the majority of its aims, there is still room for improvement. The wooden cylinders had non-uniform density as well as outer flat spots. In the future, a plastic Delrin solid object should be used in place of the wood object. Some work needs to be done to fix the wood rolling object because when it gives erroneous rolling behavior, students are presented with the wrong conclusion. This would lead to an incorrect understanding continuing throughout the quarter. 
One of the problems with the IBLA is that objects finish the race down the ramp slightly ahead of other objects. Students conclude these objects beat another when they should in fact tie. To minimize these starting effects, we recommend a shallow ramp angle (see Figure 2) and currently utilize a starting gate to ensure "fair" race starts.

There will be an ongoing process to refine worksheets and refine activities. The research team will continue analysis of student learning processes by looking at a timeline of their learning and see when they reach that 'aha!' moment. This assessment and refinement will lead to better teaching methods in Cal Poly Dynamics courses. 


\section{5: GYROSCOPE IBLA}

\section{Introduction}

In the gyroscope activity students

experimented with gyroscopes to observe precession under applied moments and concepts of angular momentum (Figure 15). Students also experimented with bicycle wheels to feel the moment applied to their hands when rotating the wheel and standing

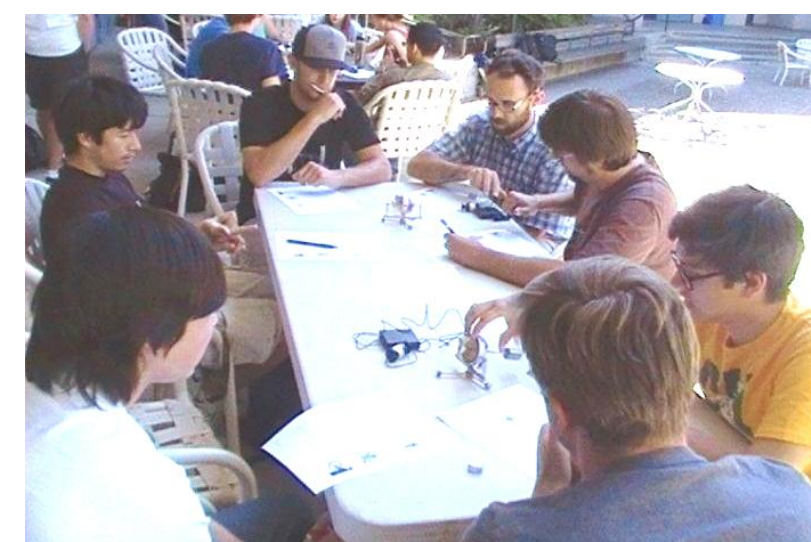

Figure 15. Students enjoying stations $1 \& 2$ and twisting on rotating platforms.

The activity was composed of four stations involving the tools of a precision gyroscope, a bicycle wheel with handles, and a lazy-susan platform. The first station had students apply a moment to a precision gyroscope and observe the resulting precession (Figure 16). The second station had students translate the gyro around the flat table to demonstrate that angular momentum is unchanged in the absence of external moments. Then they pushed on the side of the gimbal and observed the precession

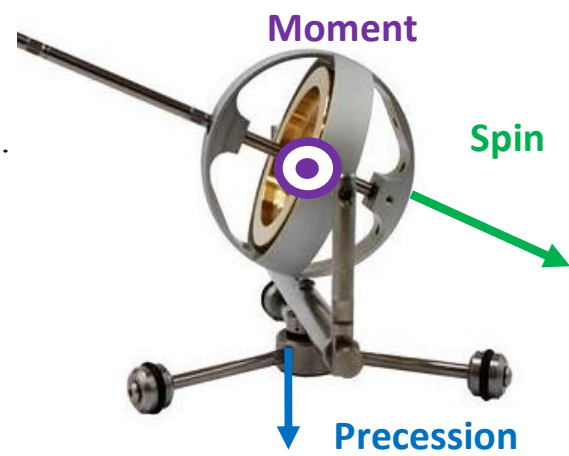

Figure 16. First station figure (similar to one of the final exam questions). On the third station, students held a spinning bicycle wheel by its two handles and yawed about a vertical axis (the spin axis is perpendicular to the outstretched arms). Next, the student held the spinning bicycle wheel by just one handle and rotated to the side (the spin axis is in the same direction as the outstretched arm).

The fourth station had students apply a roll-moment to the bicycle wheel when standing on a freespinning turn-table, as well as suspend a spinning wheel by its handle attached to a rope. 
Students made predictions before running the experiment at each station. Next, they recorded the results on a worksheet, and responded to the worksheet prompts. Through the inquiry activity, students revisited their predictions and previous ideas after seeing the physical results, and later went on to create informed conclusions.

During the hands on activity, the professor and teaching assistants walked around and checked up on student teams to make sure they were doing the activity correctly and helped to answer their questions. The instructors offered additional guidance on how to position the gyroscopes during the activity and how to set

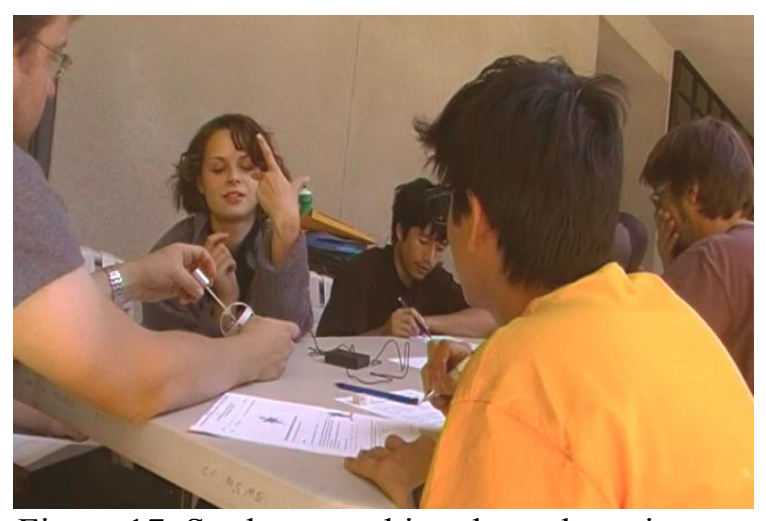
Figure 17. Students working through station two in the Gyroscope IBLA.

up the bicycle wheel on the rope.

The concepts that were addressed in this activity were the simplified gyroscopic equation (containing moment, precession, and spin) and angular momentum (magnitude and direction). One concept is shown in Figure 16; the cross-product of the spin with the moment yields the precession direction, $\vec{M}=I \overline{\dot{\Phi}} \times \overline{\dot{\Psi}}$, where $\dot{\Phi}$ and $\dot{\Psi}$ are precession and spin respectively. For example, if the spin axis is as shown, with the moment coming out at you, then the cross product of spin with moment yields the precession pointing downwards.

The concepts in this activity, 3D kinetics and kinematics and angular momentum, are important to understand dynamics. These concepts are relatively difficult for students to analyze and are not present on the DCI list. Gyroscopic behavior is very non-intuitive and challenging, thus the physical activity allows students to see the precession and spin directions and to physically feel the moments applied to spinning rotors. 
This activity was done for Intermediate Dynamics class at Cal Poly for the 2012-2013 year.

Teams of students were comprised of three to four students.

\section{Predictions review}

Students made individual predictions before each of the four stations of the activity; the results are compiled in the table below. The prediction questions and worksheet are found in Appendix C. The spring quarter and winter quarter had the same tasks to complete, but labeled the stations differently (3c-3d turned into 4a-4b). The number of stations in Spring was reduced from four to three, changing only the category titles.

Table 24. Prediction correct percentages, winter and spring 2013

\begin{tabular}{|l|c|c|c|c|c|c|c|c|}
\hline $\begin{array}{l}\text { Prediction } \\
\text { Question }\end{array}$ & Station 1a & $1 b$ & $\begin{array}{c}\text { Station } \\
2 a\end{array}$ & $2 b$ & $\begin{array}{c}\text { Station } \\
3 a\end{array}$ & $3 b$ & $\begin{array}{c}\text { Station } \\
4\end{array}$ & $4 b$ \\
\hline $\begin{array}{l}\text { Winter } 13 \\
(\mathrm{n}=66)\end{array}$ & $57 \%$ & $68 \%$ & $65 \%^{8}$ & $58 \%{ }^{9}$ & $67 \%$ & $43 \%$ & $70 \%$ & $56 \%{ }^{9}$ \\
\hline $\begin{array}{l}\text { Spring } 13 \\
(\mathrm{n}=59)\end{array}$ & $71 \%$ & $83 \%$ & $70 \%$ & $81 \%{ }^{9}$ & $68 \%$ & $48 \%$ & $44 \%$ & $58 \%{ }^{9}$ \\
\hline
\end{tabular}

The prediction scores do not really show any trends; some questions seemed to make more sense than others to students. The low prediction scores hint that there is room to better understand the concepts by the end of the class term. Student groups rotated through each station at different times - some perform the precision gyroscope station first, some perform the bicycle wheel station first. This may affect understanding because some stations may be clearer to students.

\section{Worksheet Review}

Students filled out worksheets in teams for the activity. The research team noted the common conceptions and misconceptions as follows.

\section{Fall Worksheets Response Themes:}

\footnotetext{
${ }^{8}$ Erroneous results due to directions not drawn on figure, fixed for spring '13

${ }^{9}$ Mentioned correct concepts on open ended question
} 
Student teams specified the fundamental concepts such as: angular momentum changes when a moment is applied, the simplified gyroscopic equation $(\vec{M}=I \overline{\dot{\Phi}} \times \overline{\dot{\Psi}}$, Moment $=$ Precession $\mathrm{x}$ Spin) (Figure 18), the angular impulse momentum equation $\left(\overrightarrow{H_{1}}+\int \vec{M} d t=\overrightarrow{H_{2}}\right)$, and that angular momentum keeps the wheel from falling to horizontal (rope bike-wheel station).

Students wrote different explanations on the worksheet, such as "moment applied causes angular momentum to increase, leads to precession increase." The correct idea is that

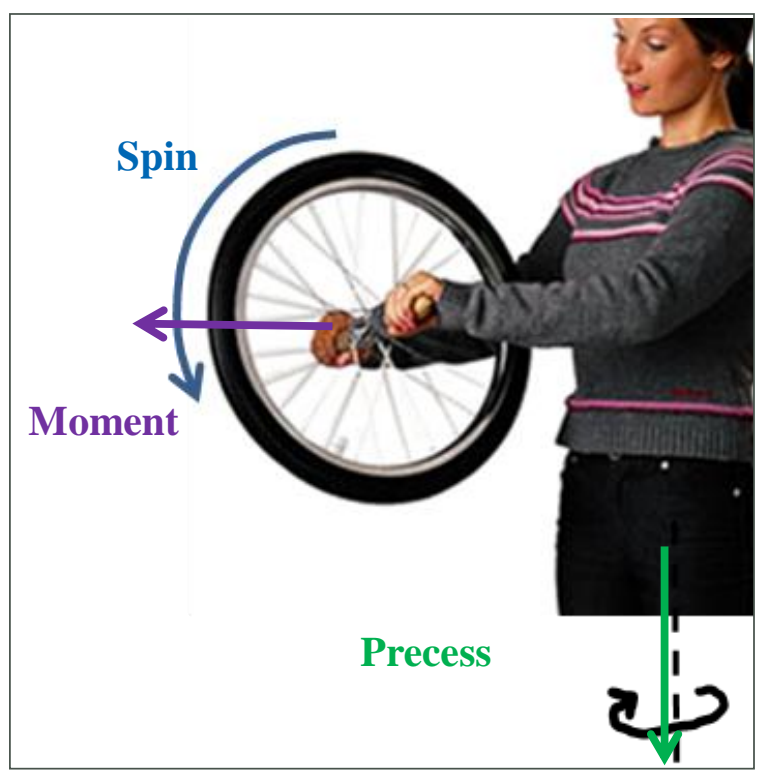

Figure 18. Precession occurs as person spins, they apply a counter moment to the wheel to keep it in place. Station 3a.

angular momentum magnitude stays constant in these experiments, and thus precession is also constant. Also, the moment causes the direction of the angular momentum vector to change, similar to how a force can cause the direction of linear momentum to change.

\section{Winter Worksheets Response Themes:}

Besides answering the worksheet questions correctly, students exhibited the following ways of thinking. Their correct concepts featured: moment causes a change in angular momentum thus leads to precession; when precession and spin point co-linearly the cross product is zero thus moment is zero; and angular momentum of objects resists changing orientation unless a moment is applied. They did a good job on writing physical experiment results, what they felt and saw from the experiment. Teams did not utilize the conservation of angular momentum equation much and focused more on the simplified gyroscopic equation. 
On the other hand, their misconceptions included: precession direction is opposite of the change in angular momentum. Similarly to the previous quarter, one misconception was not putting correct directions for moment-spin-precession axes.

\section{Spring Worksheets Response Themes:}

Students observed and explained multiple concepts, such as "moment applied to the disk causes a change in the direction of angular momentum, which causes a precession”, and that angular momentum is conserved. On the other hand, misconceptions were listed, such as 'the spin causes the rod to precess because the new angular velocity changes the angular momentum'.

The spring worksheet had spin direction drawn on the figure for station 2, which aided the analysis of the cross products. Students explained their thinking in a way that we could see what they were trying to say, by using figures and vectors.

\section{Final exam/quiz question}

Around the time of the activity, a homework assignment and quiz were given. The homework and quiz were different for each quarter, but shared the idea of reinforcing and testing the relevant concepts. The homework assignment featured an electric fan with a weight on the end to induce precession (winter '13) or the precession of a top, and the precession of a simplified space station (spring'13).

The quiz question featured a) a person standing holding a bicycle wheel pointing outwards and swung the object, find the resulting motion, and question b) find the resultant moment when an automobile's tires turn for a right hand turn (spring '13).

At the end of the ten week quarter a final exam problem was posed to the students to assess their understanding of the gyroscope concepts, which is listed next. 
The gyroscope at the right (Figure 19) has a spin direction as shown, with angular velocity in the positive $x$ direction. If you push gently on the outer gimbal in the negative $x$ direction (shown as force F), what will happen (be specific)?

The grading of the final exam questions follows the format from previous implementations:

- Score of 5 was given if students recognized that

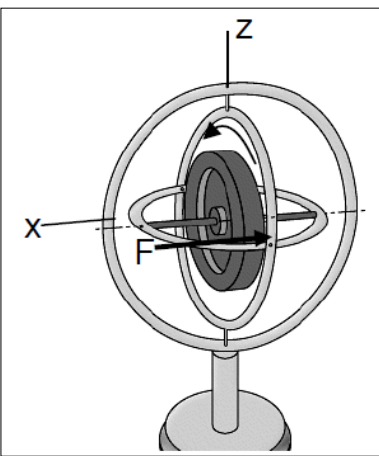

Figure 19. Final exam question the result was a precession of the disk, could apply the correct equation, and compute the axis and the correct direction of the precession.

- Score of 4 was given if students did everything, including finding the correct axis, but gave the wrong direction of precession.

- Score of 3 was given if the students recognized that the result was a precession, wrote the governing equation but then applied it incorrectly.

- Score of 2 was given if the students either recognized precession, or wrote the governing equations but not both.

- Score of 1 was given if student made a basic observation that the force caused a moment, or made some other basic observation.

- Score of 0 was given when the student gave no response or the response showed no understanding of the system.

A second problem, based on a jet engine,

(Figure 20) asks students to find the resulting

moment acting on the spinning rear-turbine

when the aircraft rotates. This problem was

modified to the context of a helicopter in

motion for the spring quarter ' 13 , and asks the

same concept. The assessment scoring is as

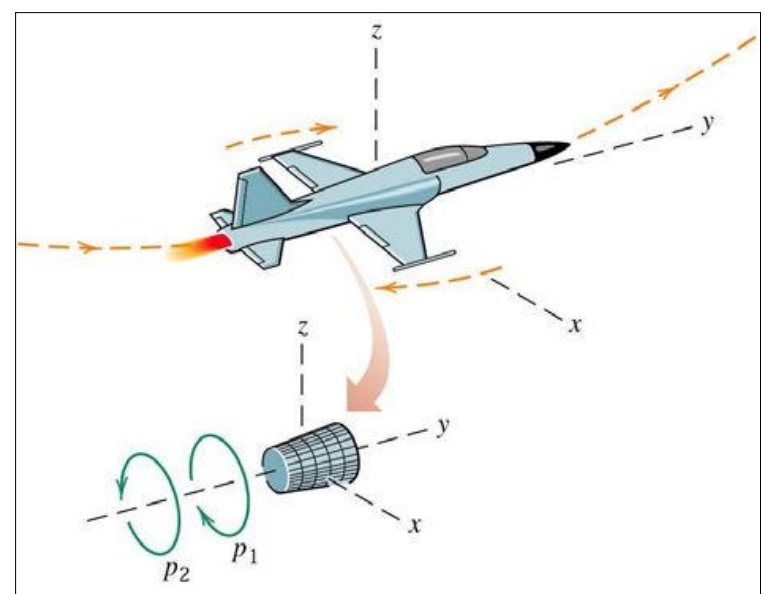

Figure 20. Final exam question two, Rotating follows: turbine in jet. From Meriam and Kraige, 2006

- Score of 3 was given if the axis and compensation decisions were both correct

- Score of 2 was given if students found the correct axis, but drew the wrong conclusion on how the pilot should compensate

- Score of 1 was given if students found wrong direction of moment and wrong direction of pilot compensation

The gyroscope activity taught in the 2012-2013 year is different from the activity in 2007 due to a different instructor and an improved worksheet. The same final exam question was asked for 
different implementations (albeit slightly different contexts for the helicopter problem). Results from the final exam questions are shown in the table below.

Table 25. Gyroscope exam question score summary

\begin{tabular}{|c|c|c|c|}
\hline Class & Quiz & $\begin{array}{c}\text { Final } \\
\text { Gimbal Problem /5 }\end{array}$ & $\begin{array}{c}\text { Final Problem } \\
\text { Jet turbine/Helicopter /3 }\end{array}$ \\
\hline $\begin{array}{c}\text { Spring '07 } \\
\text { (no lab) }\end{array}$ & - & 3.23 & 2.1 \\
\hline $\begin{array}{c}\text { Fall'07 } \\
\text { (mini-lab) }\end{array}$ & - & 3.45 & 2.26 \\
\hline $\begin{array}{c}\text { Winter '13 } \\
\text { IBLA (n=29) }\end{array}$ & - & 3.48 & 2.38 \\
\hline $\begin{array}{c}\text { Spring '13 } \\
\text { IBLA (n=69) }\end{array}$ & $6.7 / 10$ & 4.06 & 1.9 \\
\hline
\end{tabular}

The scores on the final exam problem indicate improvement on the gimbal problem, but a decrease in performance on the jet turbine/helicopter problem. The change in exam scores from spring 07 ' to fall '07 was found to be not statistically significant (from the ASEE 2008 paper [28]).

From looking at the final problem (helicopter problem in spring'13) most students found the correct direction of the rotor moment. But only a few students figured that the moment on the rotor blades is equal and opposite of the moment acting on the craft body. Some students got the symbols mixed up (spin and procession) or did not put terms into the correct units (which changes the magnitude of the answer). On the final gimbal problem, most students figured out the correct resulting precession direction; this is due to the hands on activity covering this behavior in the question.

\section{Subjective Survey}

Students provided feedback on the course, the activity, and their experiences in the subjective survey, summarized in the tables below. A Likert scale was used: $1=$ strongly disagree to $5=$ strongly agree. The activity was reported as motivating and helpful to students. 
Table 26. Gyroscope Survey Summary

\begin{tabular}{|c|c|c|c|}
\hline Class & $\begin{array}{c}\text { The gyroscope lab } \\
\text { was interesting and } \\
\text { motivating }\end{array}$ & $\begin{array}{c}\text {..helped me learn about } \\
\text { angular momentum and } \\
\text { 3d kinetics }\end{array}$ & $\begin{array}{c}\text { You should do the gyro } \\
\text { lab in future sections of } \\
\text { the course }\end{array}$ \\
\hline $\begin{array}{c}\text { Winter } \\
\text { '13 }\end{array}$ & $4.1 / 5$ & $4.0 / 5$ & $4.3 / 5$ \\
\hline $\begin{array}{c}\text { Spring } \\
\text { '13 }\end{array}$ & $4.3 / 5$ & $4.0 / 5$ & $4.3 / 5$ \\
\hline
\end{tabular}

The survey also asked when the gyroscope concepts made sense. Each number on the right column states the number of students that realized the concepts made sense for the first time, and understood the concepts afterwards. This can be seen in the table below.

Table 27. When did the concepts make sense? (Spring 2013)

\begin{tabular}{|l|c|}
\hline $\begin{array}{c}\text { First time understanding } \\
\text { concepts }\end{array}$ & $\begin{array}{c}\text { Percentage } \\
\text { Of Students }\end{array}$ \\
\hline Beforehand & $0 \%$ \\
\hline After first lecture & $\mathbf{1 8 \%}$ \\
\hline During activity & $14 \%$ \\
\hline Talking with teammates & $5 \%$ \\
\hline After activity & $\mathbf{1 5 \%}$ \\
\hline Discussion in class & $5 \%$ \\
\hline Studying it later & $2 \%$ \\
\hline After homework & $9 \%$ \\
\hline During/after post quiz & $\mathbf{2 3 \%}$ \\
\hline Still confused & $9 \%$ \\
\hline Total Class size & $(\mathrm{n}=65)$ \\
\hline
\end{tabular}

Some selected responses from students on the survey:

- "Gyros are really cool and aren't intuitive."

- "Gyroscopic motion confused me the most. Partly because it was at the end of the quarter and everything felt rushed. I always confused the moment and the precession."

- "Gyroscopic motion: It was a difficult concept to grasp because I hadn't seen anything like it before. Working through the activity definitely helped and it all seemed to click once I saw the bike wheel demonstration. "

When separately asked on the survey 'what topic in the course confused you the most?' the response gyroscopes was mentioned $25 \%$ of the time (Spring ' $13, \mathrm{n}=64$ ). 


\section{Discussion}

Overall the hands-on IBLA provided a physical experience with using gyroscopes and bicycle wheels in order to learn the relevant concepts of 3D kinetics and kinematics. Students could feel precession and moments needed to sustain certain spinning motions and witness the non-intuitive nature of precession. Students made sense of the simplified gyroscope equation and were able to apply it to new situations.

Students captured the significant concepts of the gyroscope equation and conservation of angular momentum through their worksheet responses. The data from the final exam question suggests slight improvements from using no activity to using the IBLA implementation. The instructor does not do an intervention or explanation for the gyroscope IBLA. Instead, students teach themselves by performing four stations in accordance with the inquiry principles. The subjective survey suggests the activity was helpful and motivating, and should continue in the future as reported by students.

\section{Improvements}

The worksheets will be revised with each implementation in a similar fashion to the other IBLA activities. Perhaps increasing time to complete the activity will be beneficial towards student learning. Some student suggestions for improvement are:

- "Make the activity itself more about observing what happens with gyroscopes rather than why it happens. Perhaps the explanations on the worksheet could be completed as homework after the activity rather than everyone rushing to complete them."

- "Due to the order of activities and that everyone started at different stations, the questions grew redundant. If there is a way to make it so that the most basic questions/concepts are always answered wherever you start, and then become more involved from there, it would be better for a growth of knowledge." 


\section{6: OVERALL ASSESSMENT OF IBLAS}

Here we investigate IBLAs as a whole, judge their effectiveness and make future recommendations. We also see whether IBLAs increase conceptual understanding compared to not using activities. Looking at the data, the DCI scores (pre and post) are in some instances higher and in other instances lower between the IBLA classes and those using demonstration or no activity. There was a DCI question for three of the four activities: mass-pulley, spool, and rolling cylinders, while the gyroscope did not have a DCI question. The DCI scores measured at the beginning and end of the course, along with normalized gain, are shown in the following table: This table is meant to compare performance of classes during the academic year 20122013, along with previous classes and data taken from other schools. The first column displays the class and activity given, the second and third column give the pre and post DCI percentage answering correctly, and the last column gives the normalized gain calculated from pre to post. 
Table 28. DCI scores from dynamics classes, including both IBLA and non-IBLA classes. 20122013.

\begin{tabular}{|c|c|c|c|}
\hline DCI question, class & Pre Score & Post Score & $\begin{array}{c}\text { Normalized } \\
\text { Gain*,g }\end{array}$ \\
\hline Large Public Univ., Q13 [9] & $4.6 \%$ & $56.1 \%$ & 0.539 \\
\hline Small Public Univ., Q13 [9] & $5.5 \%$ & $36.1 \%$ & 0.324 \\
\hline $\begin{array}{l}\text { Mass-Pulley, fall } \\
\text { (No IBLA) n } \sim 90\end{array}$ & $12.2 \%$ & $84.95 \%$ & 0.830 \\
\hline $\begin{array}{l}\text { Mass-Pulley, fall } \\
\text { (No IBLA) } \mathrm{n} \sim 82\end{array}$ & $15.05 \%$ & $89.16 \%$ & 0.872 \\
\hline $\begin{array}{l}\text { Mass-Pulley, winter } \\
\text { (No IBLA) } \mathrm{n} \sim 25\end{array}$ & $22.2 \%$ & $89.3 \%$ & 0.862 \\
\hline $\begin{array}{l}\text { Mass-pulley, spring } \\
\mathrm{n} \sim 30\end{array}$ & $14.8 \%$ & $93.8 \%$ & 0.927 \\
\hline $\begin{array}{l}\text { Mass-pulley, spring, demonstration, } \\
\text { intermediate. } \mathrm{n} \sim 70\end{array}$ & $43.3 \%$ & $87.3 \%$ & 0.776 \\
\hline $\begin{array}{l}\text { Spool, fall } \\
\mathrm{n} \sim 169\end{array}$ & $\begin{array}{l}\text { Rear: } 29.0 \% \\
\text { Front: } 29.0 \%\end{array}$ & $\begin{array}{l}\text { R: } 57.4 \% \\
F: 51.1 \%\end{array}$ & $\begin{array}{c}\text { R: } 0.4 \\
F: 0.311\end{array}$ \\
\hline $\begin{array}{l}\text { Spool, winter } \\
\mathrm{n} \sim 30\end{array}$ & $\begin{array}{c}\text { Rear: } 37 \% \\
\text { Front: } 33.3 \%\end{array}$ & $\begin{array}{l}\text { R: } 42.9 \% \\
F: 35.7 \%\end{array}$ & $\begin{array}{l}\text { R: } 0.094 \\
F: 0.036\end{array}$ \\
\hline $\begin{array}{l}\text { Spool, spring } \\
\mathrm{n} \sim 30\end{array}$ & $\begin{array}{l}\text { Rear: } 44.4 \% \\
\text { Front: } 29.6 \%\end{array}$ & $\begin{array}{l}\text { R: } 59.4 \% \\
F: 40.6 \%\end{array}$ & $\begin{array}{l}\mathrm{R}: 0.27 \\
\mathrm{~F}: 0.156\end{array}$ \\
\hline $\begin{array}{l}\text { Spool, spring, intermediate } \\
n=59\end{array}$ & - & $\begin{array}{l}\text { Rear: } 55.9 \% \\
\text { Front: } 47.5 \%\end{array}$ & - \\
\hline $\begin{array}{l}\text { Rolling Objects, fall } \\
\mathrm{n} \sim 169\end{array}$ & $31.3 \%$ & $89.8 \%$ & 0.852 \\
\hline $\begin{array}{l}\text { Rolling, fall, demonstration } \\
\text { UN Reno, Eric Wang, } n=60\end{array}$ & $58.1 \%$ & $55.7 \%$ & $\begin{array}{c}-0.057 \\
(* * 0.0413)\end{array}$ \\
\hline $\begin{array}{l}\text { Rolling, winter } \\
\mathrm{n} \sim 30\end{array}$ & $25.9 \%$ & $89.3 \%$ & 0.856 \\
\hline $\begin{array}{l}\text { Rolling, spring, intermediate } \\
n=70\end{array}$ & $38.8 \%$ & $88.7 \%$ & 0.815 \\
\hline $\begin{array}{l}\text { Rolling, spring } \\
n \sim 34\end{array}$ & $14.8 \%$ & $93.8 \%$ & 0.927 \\
\hline
\end{tabular}

*Normalized gains here are averaged course-wide from pre to post, not on individual matching pre to post basis. $\mathrm{G}=$ (Post-Pre) / (100-Pre).

** Different normalized gain formula used for unusual case of Pre $>$ Post. $\mathrm{G}=($ Post- Pre) / Pre. 


\section{DCI scores}

From the table results, we can see the addition of the mass-pulley activity yielded minimal improvement in DCI scores compared to that of not using the activity. As a note, the DCI question for the mass pulley is a different question than seen on the activity. The DCI question uses a constant, mass-less force, which is not seen in the activity. The scores from the interactive class are not significantly higher than the traditional instruction class; this may suggest the activity produces a small effect in learning, which may not be enough to support its continued use. Furthermore, a new activity or lecture could be used in place of this activity as a more effective use of time. But the activity may have some merits such as inducing student participation in class and providing a personal learning experience.

For the spool activity, there are minor improvements from pre to post scores in the activity classes, with no scores from the non-activity classes. There are no significant trends with the Spool IBLA DCI data. There is improvement from pre to post DCI scores for each instance of the Spool activity, but not enough to imply causation from using the IBLA. There is no significant difference in scores between the fall, winter, and spring quarters of the activity. As a note, the spool concept is not directly tested on the DCI. For instance, the question asks for friction direction on the front and rear tires of an automobile, which is related but not the same as pulling a spool with a rope and finding the friction and rolling directions. Because of the indirect testing, there may be a weaker correlation between DCI scores and learning gains from the spool activity.

For the rolling objects activity, there is an increase in pre to post DCI scores for each quarter. This signifies the activity is contributing towards student learning. There appears to be an apparent difference in the scores from the IBLA compared to the class demonstration, therefore the inquiry activity is superior in its outcomes and will continue. The rolling demonstration activity only uses data taken from one class from Eric Wang at UN Reno, so more data is needed to validate this claim. 
As a group, the IBLA activities influence the pre to post DCI scores and produce gains in student conceptual understanding in undergraduate dynamics. The average normalized gain was calculated for the three activities (considering IBLA only and beginner/Engineering Dynamics classes only) with the results: rolling $=0.86$, mass-pulley $=0.92$ and spool $=0.29$. The rolling objects and mass-pulley activity produce a larger conceptual gain than that of the spool activity, from looking at the average normalized gain G. Students tend to do better on the rolling activity than in the spool activity. This may be connected to the inability to see the friction direction when doing the spool activity. The mass-pulley activity average $G$ is slightly higher than that of the rolling cylinders, but this may be due to a small sample size and may not accurately reflect the state of student understanding. The gyroscope activity is not associated with any DCI question.

Student performance gains are not solely attributed to participation in the IBLA activities; other influences such as instructor intervention, studying, and assignments may play a significant role.

\section{Misconceptions}

Students mentally replaced their own preconceptions with experimental results in all four of the IBLA activities. Most misconceptions appeared to diminish by the time of the final exam, nevertheless some students still held onto their misconceptions. The research group did not directly test or assess for tracking misconceptions throughout the quarters.

\section{Time and Energy}

The IBLAs take time and energy to prepare, administer, and assess, in hopes of producing positive outcomes. Time is a limited resource in the classroom. Additionally the research team must be efficient in planning and time management. The IBLA activities that were put into action were the ones which teach a difficult, challenging and important concept, so the research team spent its time mostly on those items. The mass-pulley activity took around 30-40 min, spool around $30 \mathrm{~min}$, rolling activity took $50 \mathrm{~min}$, and gyroscope around $50 \mathrm{~min}$. In addition, time was 
spent outside of the activity in order to prepare, revise the worksheet, and work on improving the activity. On the other hand, performing a demonstration for each activity can take 5 minutes to 30 minutes. Based off this information, the IBLA activities are worth the time and effort that are required, resulting in benefits to student engagement and understanding.

\section{Survey}

Subjective surveys provided an opportunity for students to express their opinions on the activities. They addressed whether or not the activities were motivating, helpful towards learning the material, and important relative to other class activities. An average was taken from nine classes in which a survey was given to students. Average helpfulness of the four IBLAS was 4.18/5 on a Likert scale. Average motivating effect of the four IBLAs was 3.98/5 on Likert scale. The rolling cylinder activity was rated as more important than the spool activity on average importance (6.33 rolling vs. 6.71 spool, where lower the value means most important). Also students reported they learned the concepts from doing the activity and talking with teammates (rolling: 52\%, spool $41 \%$, and gyro $18 \%$ ). Some values are also shown in the table below:

Table 29. Average of subjective survey results

\begin{tabular}{|c|c|}
\hline $\begin{array}{c}\text { Average score of: IBLA was } \\
\text { interesting and motivating: }\end{array}$ & $\begin{array}{c}\text { Average score of: IBLA } \\
\text { helped me learn concepts: }\end{array}$ \\
\hline $3.98 / 5$ & $4.18 / 5$ \\
\hline
\end{tabular}

Based on the reported results, the IBLA activities are motivating and helpful to students from their accounts.

\section{Transfer}

Transfer is the ability to solve similar problems dealing with the same underlying concepts. Students showed transfer on the mass-pulley activity ( $70 \%$ on midterm), transfer on spool activity (65-80\% on exams), transfer on the rolling activity (50-80\% on exams), and on 
gyroscope activity ( $\sim 70 \%$ on final exams). These scores indicate students who participated in the activities understood the material to a certain degree and could transfer the concepts to a new problem context.

\section{Personal Experience of author}

From my personal experience of helping revise, design, and implement the IBLAs:

- The mass pulley teaches to most students how net force and inertia play a role in linear acceleration of the pulley systems. The activity does guide them towards seeing which dynamics parameters are important (net force and inertia) and how they affect the system behavior.

- The spool activity helps to teach the rolling behavior from the action of applied tension and friction. The results are non-intuitive to most students, including myself. From applying the FBD and KD tools, students can figure out the correct moments and forces applied. This leads to finding the correct directions and accelerations (both translation and rotation). These equations can prove why the spool rolls away from the user in the horizontal pull, which is not obvious initially.

- The rolling objects activity helps students towards understanding how hoops and solids roll in comparison to each other, with surprising experimental results. Fortunately, some groups get the concepts by the end of the exercise and go on to properly analyze rolling behavior of rigid bodies down a ramp. But not all groups smoothly pick up the concepts; they may hold onto their prior conceptions which may have a shade of incorrectness to them.

- The gyroscope activity helps students to feel the moments on the bicycle wheel handles and see the precession of the gyroscope, which are non-intuitive. Students are able to make sense of these gyro concepts and learn by doing. Though some groups yield mixed 
results in terms of understanding the concepts, most teams pick up the correct understanding.

- When students are conversing with each other, in teams, they can properly teach themselves and guide themselves towards the correct explanation of the physical results of the experiments.

- The professor interventions during the mass-pulley and spool activity seemed to have a beneficial effect on student understanding. The research team is investigating time points between cases where students pick up the concepts and when they still hold onto their misconceptions, in which an intervention can be a remedy.

\section{Conclusion}

Overall these activities are worth doing during the dynamics course and help students learn the material. We can postulate that seeing and doing the IBLA activities may help students remember the material for long periods of time. My recommendations are to continue to use and further improve the mass-pulley, spool, rolling objects, and gyroscope IBLAs. Such activities are worth the time and effort which is perhaps greater than that of demonstration or lecture. In addition, the activities contribute to student interest and motivation. 


\section{MODEL ELICITING ACTIVITIES}

IBLAs had students predict behavior of physical systems and explain the outcome. MEAs differ by having students analyze a real world problem and deliver results to a client. This provides a different way for students to learn and explore their conceptions, while improving their writing and professional skills. As discussed in Chapter 1, MEAs are built around six governing principles: model construction, reality, model documentation, generalizability, self-assessment, and effective prototype. We introduce two different MEAs in this chapter, one on gait analysis of leg motion and the other on vehicle accident reconstruction.

\section{7: GAIT ANALYSIS MEA}

\section{Introduction}

We walk with ease on our way to the office or run energetically in a sports match. Those who have experienced leg injury must take caution when partaking in these activities. Engineers contribute to those in need by performing studies on human motion and offering informed guidance. The gait analysis MEA provides a project for students to investigate human leg motion

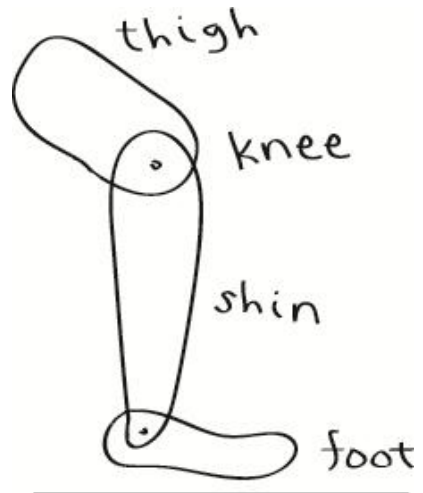

Figure 21. Leg model with limbs using rigid body dynamics and to offer rehabilitation guidance. The analysis specifically focuses on the motion of the knee, shin, and foot (Figure 21).

The MEA provides an open-ended, client-driven problem for students to solve. A fictitious sports rehabilitation company requests students to analyze various athletic motions and to submit results of dynamics quantities. The results dictate whether a set of motions are safe or dangerous and lead to recommendations for recovering patients. The required deliverables are as follows. 
- Ranking of motion activities-A procedure to rank the motions according to safedangerous conditions based on derived criteria.

- Matlab code- A script which accepts the force plate and video camera data and calculates the kinetics and kinematics at the knee joint.

- Guidelines to patients-therapists- Guidelines given to therapists to limit action of certain motions or stating certain motions are safe to perform.

- Hand-calculations - Records of free body diagrams and kinetic diagrams of the limbs.

- Plots versus time of: internal knee forces and moments, shin acceleration, anterior posterior ground reaction forces, and vertical ground reaction forces.

Students analyzed moments and forces at the knee during motion and used the results to categorize whether motions were safe or dangerous, and went on to offer general recommendations to injured patients. Students used Matlab software to input the measured data, solve relevant equations of motion, and output forces and moments at the knee joint along with other items. Teams were asked to look at a few different motions, including walking, running, jogging, using a cane, squatting, getting up from a chair, and lunging. Teams delivered their analysis to the client in the form of a memo, along with a procedure for ranking activities, a Matlab script which performed the analysis, and recommendations to patients and therapists.

The class split up the motions into groups and later recombined the results to share a large set of data. Students worked in groups of two to collect motion data, then worked with other members to analyze the data and finally write the memo.

Groups performed a set of activities while recording with a video camera and measuring force and moment data with a force plate (Figure 22). The video camera captures the position of the 'white markers', which conveys the position data of the leg joints. The man in the video in Figure 22 is performing a 'walk' motion, while stepping on the force plate with his front foot. The force plate

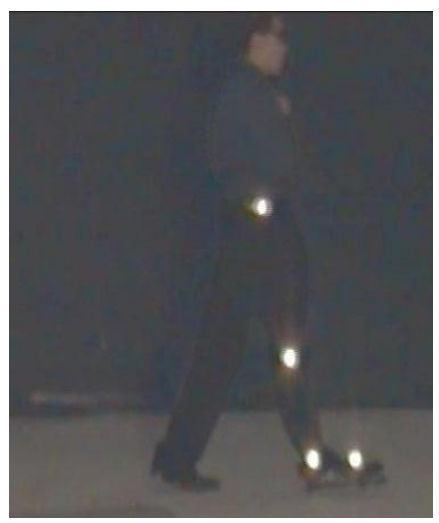

Figure 22. Video markers during motion 
measures forces and moments of the bottom of the foot vs. time. (Each motion was only analyzed while the subject had their foot contacting the force plate).

By using the measured ground reactions on the force plate along with the captured position data of leg joints, students proceeded to determine forces and moments at the knee. This is done by summing the forces and moments at each limb and setting these equal to the mass-accelerations at the center of gravity of each limb. Motions were deemed safe or dangerous in light of the calculated forces and moments. After analyzing different motions, students established a ranking or comparison between them. The student teams were free to develop their own creative ranking system and make recommendations to the client.

The gait analysis activity was designed with several aims. The activity was designed to match the MEA principles. For the model construction principle, students developed their own procedure for ranking the motions and created a computer model to analyze leg biomechanics. A sports rehabilitation company EnMotion acted as the client to fulfill the reality principle. The project featured real world contexts of biomechanics and improving human quality of life. Students stretched their previous knowledge of gait analysis and basic biomechanics as appropriate for the project, and often researched the vocabulary of basic leg limbs and ligaments around the knee. Thus the MEA taught rigid body dynamics within a real world context.

Students performed model-documentation through the memo to the client, ranking procedure, plots, Matlab code, and recommendations to patient and therapist. The generalizable principle was seen when students had to create their Matlab code with the feature to accept any data set from other groups. This allowed for collaboration amongst teams and set up a shared learning environment. The self-assessment principle was used when groups had to figure out if their plots of forces and moments were correct, as well as troubleshooting their Matlab code. Groups were able to achieve this by looking up forces and moments found in literature. One way of checking 
their calculated loads was to compare them to the value of body weight. Walking produced values around 1.4 times body weight, while jumping could be three times the normal force of body weight. There was an effective prototype of using important dynamics principles of kinematics and forces. Practical skills were also taught: digitizing markers and coding dynamics analysis into Matlab scripts can be used in future contexts.

Another aim was to use realistic technology in the classroom, including video cameras, a force plate, Matlab software, and video conversion/editing software. As a reminder, the video camera captured position data of the leg joints and the force plate measured forces and moments between the active foot and the ground.

Students progressed through this project by performing dynamics analysis on the leg during motion. To provide engineering analysis for the client, students calculated forces and moments acting on the knee joint (

Figure 23). This was done through a process called 'inverse dynamics', by starting analysis with the foot and working towards the knee. The FBD and KD were used to determine the equations of motion.
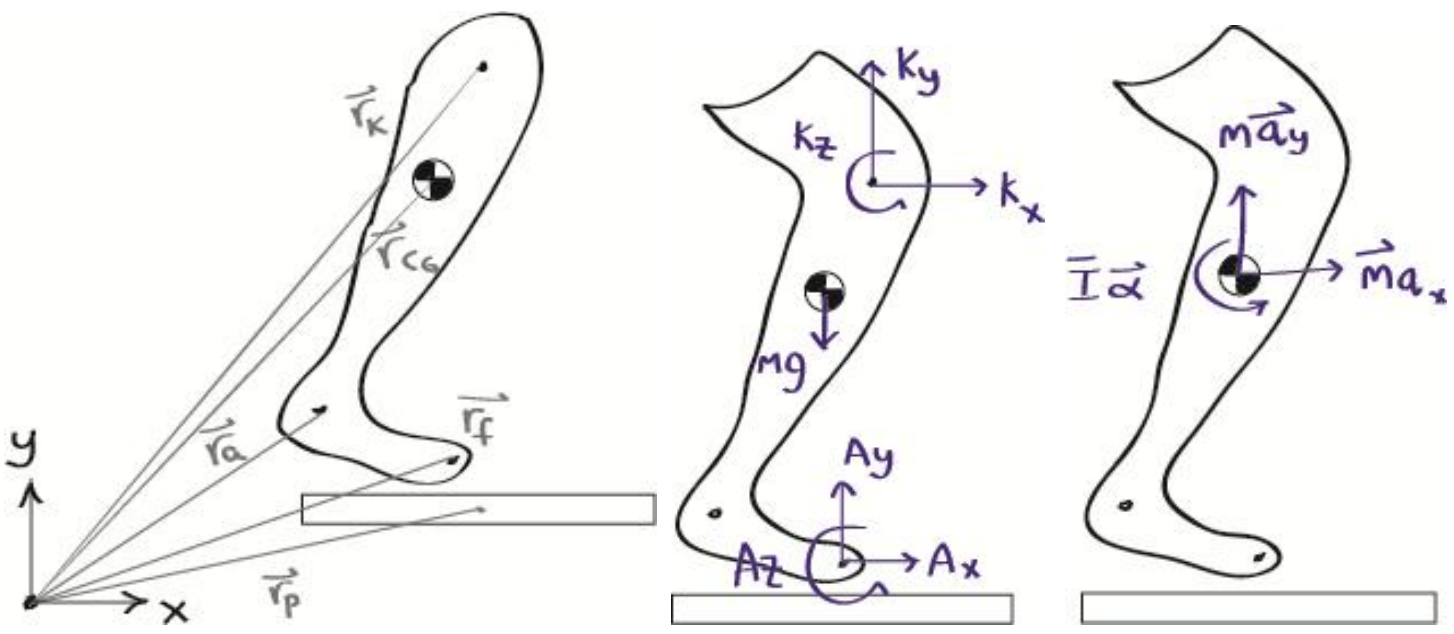

Figure 23. Position vectors, Free Body Diagram, and Kinetic Diagram of lower leg 
In this problem setup, the student is given: $\mathrm{Ay}, \mathrm{Ax}, \mathrm{Az}$ from the force plate, while the joint positions from the video camera are differentiated to get accelerations. The task is to find the loads at the knee: $\mathrm{Ky}, \mathrm{Kx}, \mathrm{Kz}$. The equations of motion are as follows:

Sum of $\mathrm{x}$-forces on lower leg:

$$
\sum \overrightarrow{F_{X}}=\overrightarrow{A_{x}}+\overrightarrow{K_{x}}=m \vec{a}_{G x}
$$

Sum of y-forces on lower leg:

$$
\sum \overrightarrow{F_{Y}}=\overrightarrow{A_{y}}+\overrightarrow{K_{y}}-m g=m \vec{a}_{G y}
$$

Sum of z-moments about the center of gravity on lower leg:

$$
\sum \vec{M}=\vec{M}_{z}+\left(\overrightarrow{\mathrm{r}}_{\text {Ankle } / C G} \times \vec{F}_{\text {Ankle }}\right)+\left(\overrightarrow{\mathrm{r}}_{\text {Knee } / C G} \times \vec{F}_{\text {Knee }}\right)+\vec{M}_{k z}=\overrightarrow{\mathrm{I}}_{G}
$$

The gait lab used a simplified ${ }^{10}$ leg model (

Figure 23). The model assumed motion was primarily in the 2D sagittal plane, and considered out-of-plane forces and moments (Fz, Mx, My) to be negligible. Even though this model made assumptions, it provided a significant platform for students to analyze athletic motion and obtain approximate values for the loads at the knee.

\footnotetext{
${ }^{10}$ This model makes an assumption that the loads on the knee joint are primarily taken by bone, while the tendons (ACL, CL) which support some of the load, are ignored.
} 


\section{Detailed Steps for Activity}

An overall view of the data manipulation from the activity is shown in the following figure.

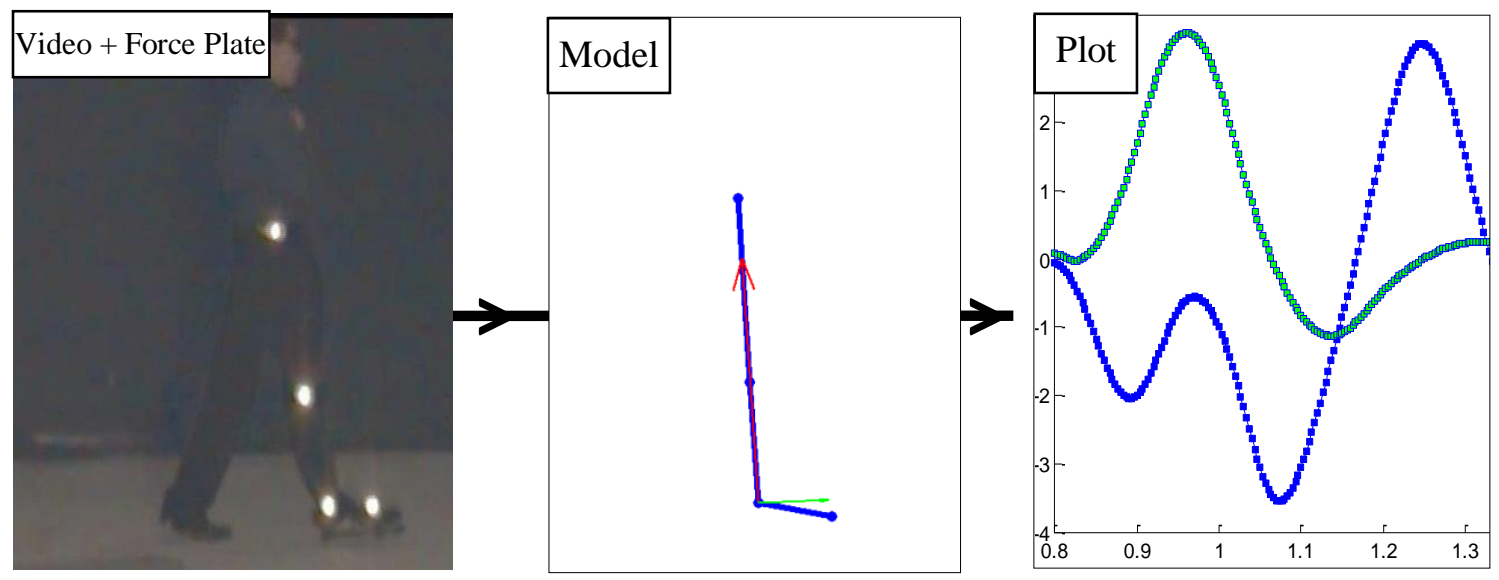

Figure 24. Data analysis progression: Record Video footage and collect force plate data, then digitize joint positions into a Model, then use Matlab to solve for loads and plot results.

The first stage was to perform the motion, record with a video camera, and then digitize the markers. In this task, the user placed their cursor on the white dots in the video then had the program export position coordinates. The marker digitizing was performed with a Matlab script 'Digitizing Tools,' written by the Hendrick Lab ${ }^{11}$ at UNC. This exported position data which was then read into the gait analysis script as a text file. The digitized marker positions were converted from pixels into meters (or feet). Students were given a packet containing some tutorials and engineering instructions to help them with these tasks.

The next step was to measure force plate data during motion and record it as a text file. The force plate captured force and moment data in all three directions. The directions on the force plate were determined and transformed to the standard axis coordinates of the analysis.

After the video and force plate data were collected, the data were imported into Matlab, where it was edited and used in dynamics equations (stage two). Teams solved the sum of forces and

\footnotetext{
${ }^{11}$ The Hendrick Lab at http://www.unc.edu/ thedrick/software1.html. We offer a great thanks to the Hendrick Lab for creating this tool which makes our MEA possible.
} 
moments equations, along with rigid body kinematics, on the shin, foot and thigh. In the process, they needed mass properties of the limbs, which were provided in a handout ${ }^{12}$. Teams made use of reactions at the foot and accelerations of the shin and foot center of gravity to get forces and moments at the knee joint. The knee forces were transformed into axial and shear components to be more relevant to the analysis. Then students coded the equations into a Matlab script to determine the results using the input data. See the following flow chart for the Matlab steps.

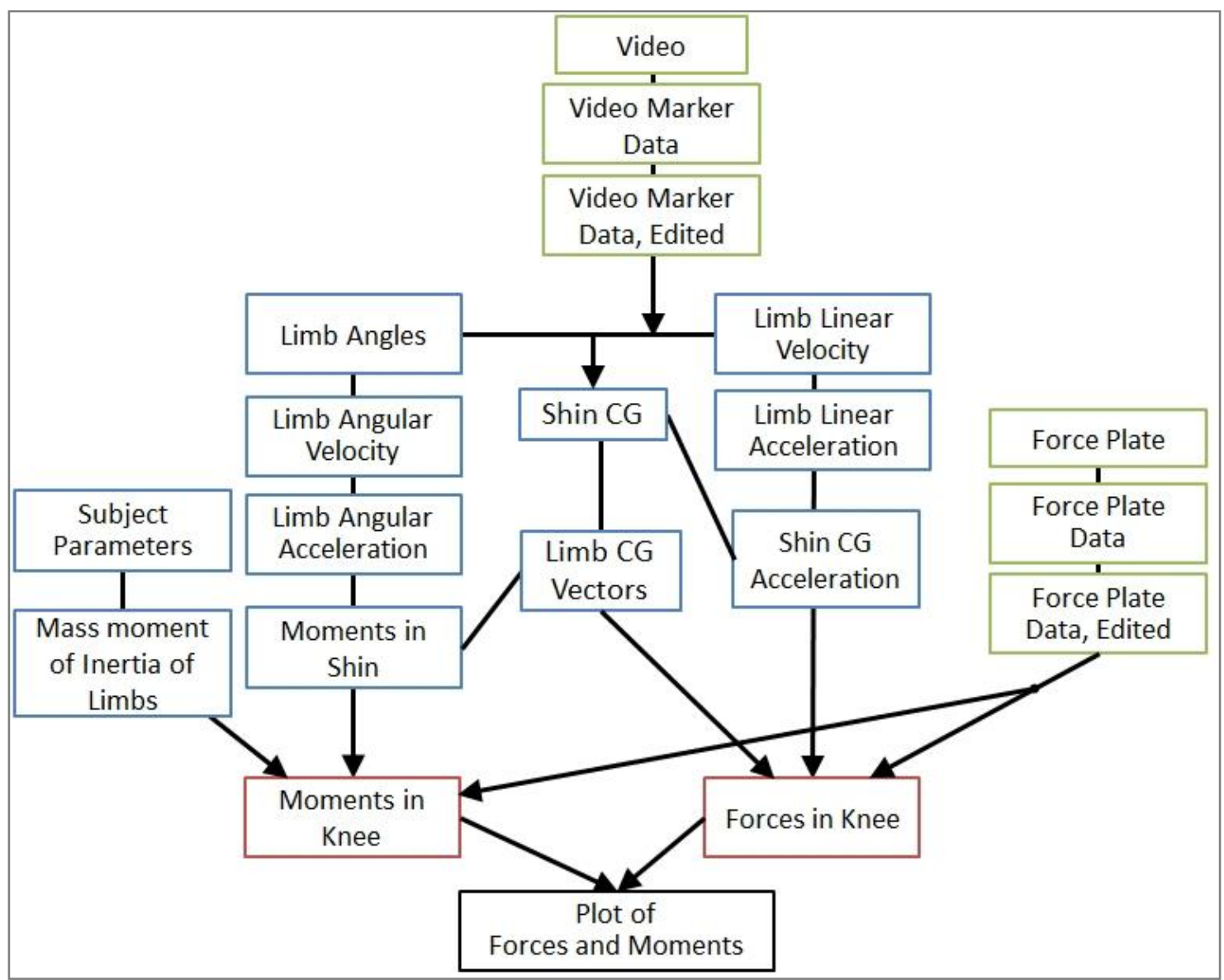

Figure 25. Flowchart of measuring motion data and solving for moments and forces in the knee Students then used the dynamic loads at the knee joint to develop a ranking method for the motions (stage three).

\footnotetext{
${ }^{12}$ Dempster's Body Segment Parameter Data for 2-D Studies [37]
} 
In weekly lab sections, students progressed in the project, the instructor presented new tasks, and teaching assistants aided in answering student questions. Students practiced and learned appropriate Matlab commands and skills in weekly lab assignments which were related to the MEA. The professor would assign a task to target a necessary activity skill. For example, one task was to "find the velocity of the center of gravity of a limb joint". This step-by-step process enabled the students to perform analysis using Matlab and check their work along the way. This guidance eased the students into feeling comfortable about the challenging work they needed to perform. Students developed Matlab skills for this project: matrix manipulation, video marker capturing, data smoothing, and limb plotting. These skills were useful to the gait analysis and fortunately can be applied to future projects. The project for the intermediate dynamics class took place over three to four weeks, and ran for the winter and spring quarters in 2013.

\section{Assessment}

As a reminder, students delivered a memo to the client, along with results in the form of plots and load values at the knee. The author assessed students' work by looking at the six MEA principles. Students created a procedure, so the author reviewed the type of ranking and counted the number of motions ranked. The author also reviewed how they addressed the client and the quality of recommendations to the patient-therapist. One item that the author checked was whether students normalize their loads to body weight. Another item was to see if they had a generalizable procedure and to observe future ranking capability. The author also inspected how students presented their results to connect to a realistic context. These assessment criteria produced the winter-spring combined results below.

\section{$\underline{\text { Values of forces and moments }}$}

Groups produced reasonable values of moments and forces at the knee joint. One can normalize forces to the patient's body weight in order for data to be compared to other test subjects. In winter, only a few groups (5.3\%) normalized their forces and moments to the subject's body 
weight. This improved in spring quarter, when most groups $(80 \%)$ normalized by the subject's body weight. This was suggested by the instructor. Also, a few groups $(10 \%)$ in spring normalized their moments by (body weight $) *($ leg length), allowing for a similar comparison of moment results in light of varying patient leg length.

\section{Number of motions ranked}

The minimum number of motions required to analyze was four. In winter, 15/19 groups ranked 5 or more motions (average was 5.05 motions). In spring, 22/30 groups ranked 5 or more motions (average was 6.13 motions). Thus, groups were looking at a wide range of activities ranging from walking to lunging to jumping in order to conduct a comprehensive investigation.

\section{Ranking Method}

Next, students groups created various ranking methods to relate safe and dangerous motions for the patient (seen in the following table). Students' choices of ranking showed they were thinking about how different motions and loads affected the human body in a realistic context of biomechanics.

Table 30. Summary of ranking methods. The left column displays the type of ranking used, the second and third columns count the number of groups that represented such method, in the winter and spring quarters.

\begin{tabular}{|l|c|c|}
\hline \multicolumn{1}{|c|}{ Ranking Tally Criteria } & $\begin{array}{c}\text { Groups } \\
\text { in Winter }\end{array}$ & $\begin{array}{c}\text { Groups in } \\
\text { Spring }\end{array}$ \\
\hline Compared motion based on one or two quantities & 8 & 6 \\
\hline $\begin{array}{l}\text { A small number of groups ranked their motions based upon a single-dimension criterion. } \\
\text { Some single-dimension ranking schemes used a cutoff value - where motion values beneath } \\
\text { the threshold are still considered safe. Most groups used a combination of two parameters to } \\
\text { rank their motions; parameters include: axial knee force, shear knee force, knee moment, the } \\
\text { duration of the moment/forces, the rate of change of the loads/moments, the moment when } \\
\text { the knee-shin angle was close to perpendicular (90 degrees), and the maximum } \\
\text { force/moment. }\end{array}$
\end{tabular}


Compare motion loads to loads of walking

0

3

Three groups compared the motion results to that of walking, because walking was considered a baseline and a safe activity. For example, jumping created a moment that was three times that of walking, and thus was considered dangerous.

\begin{tabular}{|l|c|c|}
\hline \multicolumn{1}{|c|}{ Looked at Max or Peak values } & 19 & 30 \\
\hline $\begin{array}{l}\text { All of the groups used maximum values from the loads at the knee. The max values can be } \\
\text { negative due to compression. }\end{array}$ & 0 & 1 \\
\hline Looked at Average Values & $\begin{array}{l}\text { One group used the average load value to determine which motions were safe over long } \\
\text { durations of time. }\end{array}$ \\
\hline
\end{tabular}

\begin{tabular}{|l|c|c|}
\hline Separate ranking for each type of injury & 3 & 7 \\
\hline A few groups connected the idea that ranking should be based upon injury type, whether it is \\
injury from muscles, tendons, or bone. For example, moments can cause injury to muscles \\
and tendons, shear loads can cause injury to tendons and ligaments, and normal loads at the \\
knee can cause injury to the leg bones. Therefore each motion was ranked three separate \\
ways according to the separate type of injury it produced.
\end{tabular}

\begin{tabular}{|l|c|}
\hline $\begin{array}{l}\text { Categorized into safe-moderate-dangerous bins, (by criteria such } \\
\text { as safety factor or load) }\end{array}$ & 3 \\
\hline $\begin{array}{l}\text { Some groups ranked motions by using a series of ranges with upper and lower limits to each } \\
\text { range, creating a tiered list. An example of this method uses the ranges of safe, mild caution, } \\
\text { and dangerous to categorize each motion. For example, factor below } 5=\text { safe, and between } \\
5-10=\text { moderate, and greater than } 10=\text { dangerous. }\end{array}$ \\
\hline
\end{tabular}

Composite Score or Equation 4 8

Some groups created composite rankings or weighting factors to rank the motions from a combination of parameters. Weights were assigned a value based upon the significance of an injury to a certain part of the leg. For example, to build a composite score out of weighting factors one group created a danger score, reminiscent of a safety factor. Danger Score $=$ Force shear ${ }^{*} 0.3+$ Force axial ${ }^{*} 0.4+$ Moment $* 0.3$. Groups used different choices of equations, such as using the square root of certain loads. 


\begin{tabular}{|c|c|c|}
\hline Subjective combination of normal, shear, moment & 3 & 3 \\
\hline \multicolumn{3}{|c|}{$\begin{array}{l}\text { Some groups compared the loads from different motions and made a subjective ranking, } \\
\text { without any objective criterion. }\end{array}$} \\
\hline No ranking given, defer to therapist & 0 & 1 \\
\hline \multicolumn{3}{|l|}{$\begin{array}{l}\text { One group presented the load results and deferred the ranking task } \\
\text { to the physical therapist, citing that the therapist is the expert on } \\
\text { the matter. }\end{array}$} \\
\hline (Total) & (19) & $(30)$ \\
\hline
\end{tabular}

*Note, there is overlap between categories, i.e. groups may have ranked their motions in ways which fell under more than one criterion in the table.

$\underline{\text { Rank future activities }}$

Students could generalize their ranking system to handle future cases and new motions, extending the applicability of their analysis to future contexts. To the professor's dissatisfaction only $3 / 19$ groups in winter provided a mechanism to rank future activities. Fortunately, in spring, 23/30 groups explicitly allowed for ranking of future activities.

\section{$\underline{\text { Reasonable numbers }}$}

The winter and spring submitted force and moment values appear to be reasonable. Only a small fraction of the groups had errors in their code which slightly affected the magnitude of the results. The winter and spring students all submitted plots that appear to be reasonable.

\section{$\underline{\text { Recommendations }}$}

The next assessment was to review how and what students recommended to the patient and therapist. Students could offer advice as well as an injury recovery plan. Recommendations from spring and winter varied from a step-up plan from light activities to strenuous activities, to detailing which actions are safe in certain situations. In spring some common themes were to 'move up from less strenuous activities to more strenuous activities in a gradual manner' as well as 'progress from safe to moderate to dangerous activities' and to 'avoid certain motions that put undesirable stress on the leg.' A more specific example stated that one should 'avoid skeletal 
injuries in the shin or knee joint and thus stay away from high impact activities, and for muscular or ligament injuries stay away from activities that involve high muscle usage or that require extensive amounts of leg strength.' Student groups addressed the patient and therapist in different ways and most gave acceptable answers.

The author also assessed the MEA results by way of addressing the six principles.

\section{$\underline{\text { Reality }}$}

The reality principle of MEAs was used in the professional and sports-injury context. Most groups addressed their memo to the client and patient athletes by using appropriate language and tone through the documents.

\section{$\underline{\text { Self-Assessment }}$}

A decent amount of winter groups (10 out of 19) commented on their data quality (including data noise) and practicality when reporting to the client. Students compared the magnitudes of the resulting forces, or commented on possible sources of error, of the motions under question. The professor checked plots to give students feedback a week before the final turn-in (plots of knee internal forces and moments, and of shin CG acceleration), for the first run of the gait lab.

Some groups did well in explaining any discrepancies in their results and hinting at possible sources of error and paths towards improvement. In Spring, (15 out of 30) groups self-checked their model for practicality in different ways. One group stated they validated their position data by looking at the motion plot of the limb as a frame-by-frame display and making sure it was displaying correctly. Other groups noticed sources of error in their code, such as how their program imports data and uses it. A common student response was that the engineers are not physical therapy experts, therefore only those with expertise in the field should interpret the data results. Other groups stated that 'the data is approximate and based off experiments that could 
contain errors' and that 'repeating the motions multiple times could improve the credibility of the results.' Students should have checked online resources to look at the internal loads, but only a few groups did this.

\section{$\underline{\text { Generalizable Model }}$}

The next assessment looked at how groups made a generalizable model. In winter, very few groups $(11 \%)$ created a generalizable computer code for their motion analysis work; instead, they created computer code for dealing with a single given motion at a time. Only a few groups mentioned that their code could be run to look at future motions and that the ranking criteria could be modified if different reasoning were to be chosen. Modifications were made in the assignment in spring, so most groups ( $>90 \%)$ made a generalizable model with their Matlab code. Their code could accept any input file (force and position data), subject parameters, and then output forces and moments at the knee joint and display corresponding plots of loads and accelerations of the shin.

\section{Effective Prototype}

The effective-prototype principle was manifested in students gaining experience with 2D motion analysis applied to biomechanics, and gathering and interpreting real world data. As stated earlier, the professors prefer that students are challenged to gather and analyze real world data to enrich the learning experience, rather than being given the motion data. In spring, different groups produced versions of code that enacted essentially the same action as other groups, but used different routines and methods.

Students can extend their knowledge of rigid body dynamics of the human leg to analyze forces and moments of a robotic linkage or new motion activities like climbing up stairs in future contexts. 


\section{Extend to Realistic Context}

In spring, many groups extended the model to a realistic context and made connections to reality. This was done by explicitly identifying the physiology of the leg and knee, including tendons, ligaments, and bones and by doing additional background research, which they, in some cases, connected to their results. Students researched topics beyond the requirements of the client memo. One group stated "there are several areas this study could be taken to become a more solid basis for physical therapists and people of related fields." They also mentioned that "numerous other extremities, positions, and activities could be analyzed to create a reputable source of kinematic and kinetic data of the human body."

\section{Survey}

To foster project feedback and improve the activity, a subjective survey was given a few weeks following the activity. The subjective survey details how participants felt about the difficulty of the lab as well as detailed steps of the lab. Comments from the survey stated that the gait lab MEA was challenging, short on time, fun, and interesting.

Table 31. Gait Activity Survey results from winter quarter, last entry is from spring quarter

\begin{tabular}{|l|c|}
\hline \multicolumn{1}{|c|}{ Response: } & $\begin{array}{c}(0=\text { strongly disagree } \\
\text { to 5 = strongly agree })\end{array}$ \\
\hline Analyzing the physical motion in kinesiology lab was interesting & 4.3 \\
\hline $\begin{array}{l}\text { I would prefer that we were just given the data and not gone to } \\
\text { the lab }\end{array}$ & 2.0 \\
\hline There was not enough guidance provided during the gait lab & 3.3 \\
\hline You should do the gait lab in future sections of the course & 1.6 \\
\hline The difficulty of the gait lab was (0 = too hard, 5= too easy) & 3.8 \\
\hline The project helped to learn dynamics (Spring quarter) & 4.0 \\
\hline
\end{tabular}


Additional Spring comments were as follows:

- Ifelt that the lab activities directed attention from dynamics. The focus on the project became coding, technical writing, and teamwork, while the dynamics was the easy part.

- $\quad$ The Matlab projects could use a little more direction, but I realize they are supposed to be like "real life"

- $\quad$ The gait lab was a much larger task than what much of the class assumed it would be. It was difficult to understand what material you desired in the final report.

Students found the kinesiology work interesting, liked to gather data in real life, and wished the gait lab be run again in the future. On the other hand, students stated they were not given enough guidance during the project, and found the gait lab difficult. Students mentioned that the activity guidelines were often vague and open ended.

The gait lab was difficult to students for many reasons. The professor purposely made the project rather ambiguous (information was given on the client memo), and did not tell students how to do every part of the lab, rather he let them figure it out. Another reason for difficulty in the gait lab was that it was the first time running the activity so things did not always go smoothly. The computer coding in Matlab proved to be a substantial challenge to the students, more so with those whom did not possess adequate experience with Matlab. This project was relatively challenging due to the quantity of procedural steps to analyze the video and force plate data. Students were often underprepared for the coding and thus learned as they went along. Another challenge was analyzing real world data because the signals were noisy and needed to be filtered and interpolated (the force plate data was captured at ten times the rate of the video data, so the data had to be interpolated). Another aspect was making xyz coordinate systems match between the force plate and FBD of the leg. 


\section{Discussion}

Students commented on the difficulty due to time constraints and extensive Matlab coding, but also mentioned the activity was fun and interesting. In the Spring quarter, more groups created a ranking system with the capability to accept future motions (an increase of $60 \%$ ), which may be due to enhanced guidelines and directions. Overall students produced a good set of work in the second implementation of the gait analysis activity.

Students improved their conceptual knowledge of rigid body dynamics in this activity. Most groups did correct free body diagrams, kinetic diagrams, and rigid body equations; these fundamentals are the core of the curriculum and should be correct before proceeding through the gait lab project. They were able to apply rigid body kinematics to a problem based on human limbs in motion, to calculate acceleration and forces, and also angular accelerations and moments.

While working in teams, they were able to record real data, manipulate it, and analyze the results with a ranking procedure. They were able to extensively use Matlab software to manipulate data with their own coded scripts. They traversed from data acquisition in the kinesiology lab to data manipulation to analyzing results and making decisions based upon their data. Students gained knowledge in the field of biomechanics from researching various sources and applying that knowledge to the project.

Students brought similar and unique solutions to the table in their ranking methods. By communicating with a client, students practiced their writing and professional engineering attitudes and behavior. Hopefully students took away a realistic experience with them along with skills in real data manipulation.

\section{Improvements}

The gait lab is a fairly new project; it has only run for two quarters so there is room for improvement. After the first implementation, there was a change to the foot analysis - the motion 
of the foot can be considered negligible (foot has small mass, small moment of inertia, is relatively flat on ground). Therefore foot loads can be considered to be located at the ankle.

The mp4-avi video conversion can be performed by the professor or TA outside of lab time, to provide the students more time on important aspects of the project. The students could have intermediate turn-ins, such as making a Matlab function to take the numerical derivative, working with 'xlsread', or writing other example code. In order to help with future implementations of the Gait Analysis MEA, the author wrote a full code (see Appendix G). 


\section{8: VEHICLE ACCIDENT RECONSTRUCTION (VAR) MEA}

\section{Introduction}

Students applied their understanding of work-energy and impulse momentum through the role of a scientific investigator analyzing vehicle accident reconstruction cases. Their task was to analyze four accident cases using dynamics principles to conclude whether the drivers were at fault, presumably due to speeding, and whether prosecution was recommended. Beyond those four cases, they designed a procedure for police officers to collect data on site and created an Excel program to solve for initial car velocities. Students delivered the completed tasks to the client, the Sri Lankan police department. The client initiated this project by providing a memo with an introductory note and four accident cases for students to review. Students delivered the following items:

.... (1) an Excel program that we can use to estimate driver speeds for almost any accident scenario, (2) a User's Manual that teaches the basic principles of accident reconstruction to our officers and provides instructions on how to use the program, (3) instructions on what parameters the officers should collect at the scene, (4) your detailed analysis on how you used your program to solve each of the accident scenarios and if you think we should prosecute the drivers. This memo should also include a discussion on if you think we should prosecute the drivers in each of the scenarios, especially given the uncertainty in some of the values used in your analysis (e.g., our friction coefficients can vary by $10 \%$ in many cases).

The main dynamics concepts of this project were impulse momentum and work-energy. In a car collision, momentum is conserved, but total mechanical energy is not conserved (energy is converted into heat and sound that are not easily calculated). Students were expected to apply impulse momentum and work-energy at correct phases of the vehicle crash. Impulse momentum was used during a collision of two vehicles to relate the velocities and angles immediately before and after the collision. Work-energy was used to find the amount of kinetic energy lost during skidding and impact. Students solved the four cases with pencil and paper, and went on to verify their answers with Excel software. The following equations were utilized: 


$$
\begin{gathered}
\text { Work-energy }^{13}: \quad K E_{1}+\text { Work }_{1-2}=K E_{2}+\Delta P E \\
\text { Impulse momentum: } m \overrightarrow{v_{1}}+\int F d t=m \overrightarrow{v_{2}}
\end{gathered}
$$

The Vehicle Accident Reconstruction activity addressed relevant MEA principles, including: model creation, reality, self-assessment, model documentation, generalizability, and effective prototype.

- Student groups created their own models of accident reconstruction, a procedure to administer the method and collect vehicle parameters and crash scene details, and an Excel program.

- They handed their documentation off to a 'realistic client,' the Sri Lankan Police department. This activity represented real life situations because law enforcement officers routinely perform accident reconstruction. The vehicle crash cases were modeled after realistic cases ${ }^{14}$.

- Students self-assessed their work when comparing their hand calculation values to computer calculations in Excel, as well as when thinking about uncertainty in answers due to input ranges.

- Students documented their model in the procedure, Excel program, and user manual which were given to the client.

- The vehicle accident models were generalizable because they had to accept any vehicle parameter, speed, distance, and angles in order to apply crash reconstruction in future cases.

- Students made use of the work energy equation and conservation of momentum principles, which are major concepts in dynamics, to satisfy the effective prototype principle.

This activity has run multiple times since its development in 2008. This document focuses on two implementations for Engineering Dynamics in winter and spring of 2013.The specific accident cases have been slightly revised and can change from quarter to quarter. The current accident cases from the 2013 year are as follows:

\section{Accident Cases}

The four accident cases are shown as A, B, C, and D.

\footnotetext{
${ }^{13}$ Work $_{1-2}$ consists of non-conservative work due to kinetic friction or bumper crushing

${ }^{14}$ Teresa Oggletree and the Oceanside Police Department
} 
- Case A featured a single car which lost control and skidded for a distance, flipped over and skidded on the roof, then hit a pole and crushed the front bumper by a given amount.

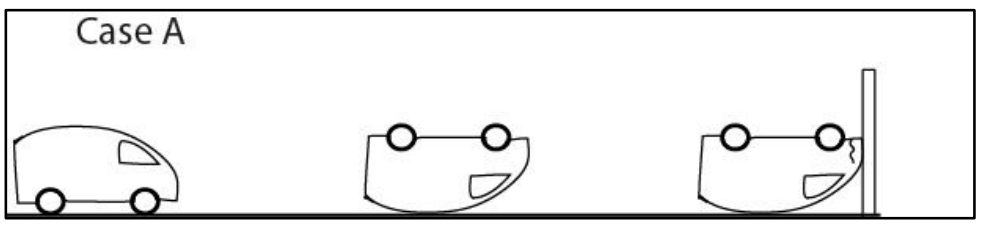

- Case B featured an oblique collision of a car and a cement truck, given initial velocity range of the truck and impact angles.

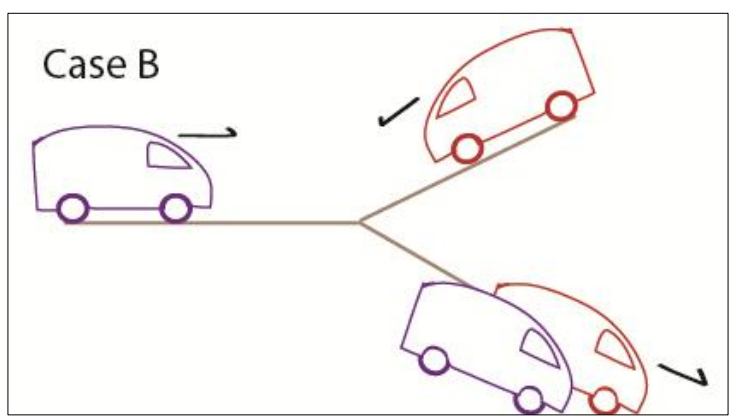

- Case $\mathrm{C}$ featured two cars colliding on an inclined road where they both skidded different distances afterwards.

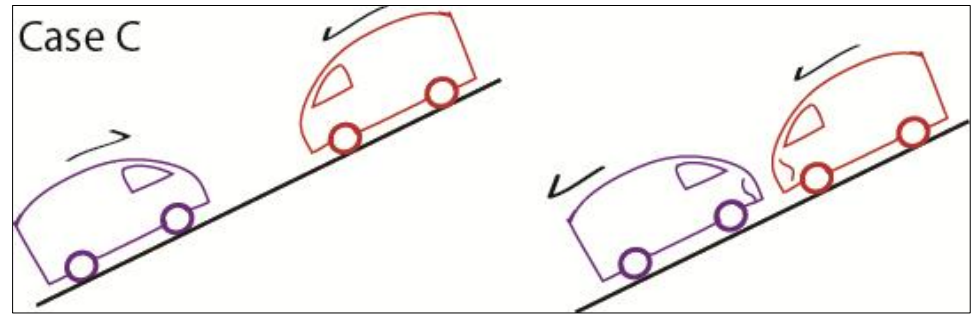

- Case D featured a collision between two vehicles when one car merged from an onramp, and afterwards the vehicles skidded off at oblique angles.

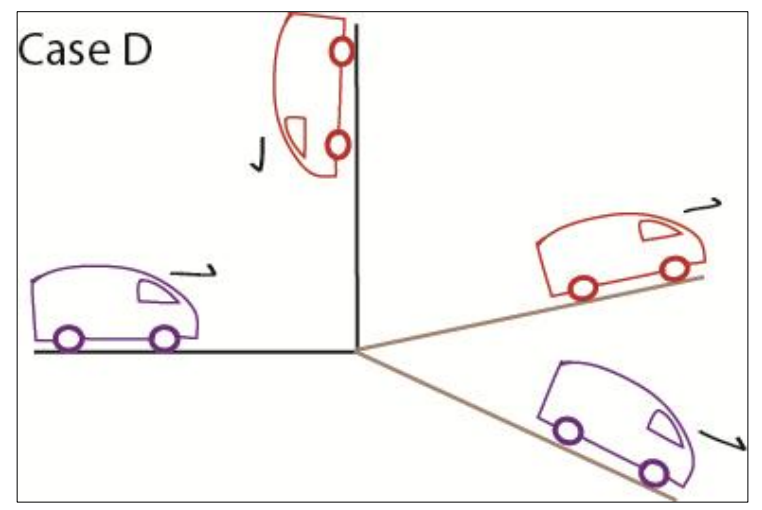

The students were given a homework assignment prior to the MEA in which they solved the accident cases by hand, some cases individually while other cases in groups. They had one to two weeks to complete the project by writing a memo, providing hand calculations, making a user 
manual, and creating an Excel file to perform accident reconstruction. They were also given a subjective survey at the end of the quarter to garner feedback.

Student teams were assessed from the following list: addressing the client in a realistic and professional tone; correctly applying the momentum and energy concepts; self-checking their model for accuracy and certainty; obtaining reasonable values and whether they recommend prosecuting the driver; providing a generalizable Excel program; and making the user manual user-friendly. The results of these assessments are as follows:

\section{Results}

\section{$\underline{\text { Client }}$}

In winter, 7 out of 7 teams wrote the memo to the Sri Lankan client with appropriate tone and thus took the premise of the project seriously, some groups more than others. Most spring groups (6 out of 9) addressed the Sri Lankan client sufficiently in the written memo. Those that did not instead addressed the professor in the delivered memo.

\section{Conceptual Understanding}

All of the winter groups (7 out of 7) demonstrated correct conceptual understanding of impulse momentum and work-energy. Some teams went on to explain in detail how in each case these engineering fundamentals were applied. All spring teams ( 9 out of 9 ) demonstrated conceptual understanding of work-energy and impulse momentum. Work-energy can be applied when the vehicles skid, flip over, or crush the bumper. When two vehicles collide the impulse momentum equation can be applied (momentum is conserved). Some groups had some errors in the hand calculations, forgetting about the work done by bumper crushing or change in potential energy.

The spring professor commented on students reports. He corrected one group by stating that friction in an impact is not negligible (cannot use 'e' here). In other words, it is not a good approximation for oblique collision to say momentum is only conserved in the normal direction, 
but not the tangential direction. He also reminded students that the conservation of momentum equation uses velocity vectors.

\section{Model Creation}

Students developed the model of the crash situation and procedure, and decided which factors they took into consideration. Students took into account factors from the physical world (how the crash happened, the weather conditions, driver condition) to analyze the problem and figure out what parameters were critical. Students were given parameters when investigating each accident case (final velocities, skid distances, vehicle masses) and even extra information like the time of day and temperature conditions.

When using their Excel program to find velocities and to decide upon driver prosecution, they experimented with different parameters to justify their prosecution decisions.

\section{$\underline{\text { Self-Assessment }}$}

As a note, the initial prompt purposely set up the students to think about uncertainty when it provided ranges for some given initial velocities and also given velocity direction angles.

The winter groups (7 out of 7) stated evidence of uncertainty concerning the initial vehicle velocities in their reports. This uncertainty was due to some of the given data being given as ranges. Because the input data was given as ranges, the output data should include ranges. Students stated uncertainty in different ways, for example, in the form of percentage of error (sometimes $10 \%$, or $\pm 5 \mathrm{~km} / \mathrm{hr}$ ). This stemmed from friction coefficients between a vehicle and the given road, crush coefficients of the front bumper, or angles of vehicle travel.

Most spring groups (6 out of 9) stated reasoning for uncertainties in their analyzed velocity values, which showed the self-assessment principle. The remaining 3 out of 9 groups only wrote the velocity answers with no uncertainty consideration. Students used creativity and engineering judgment to demonstrate self-assessment. For example, students found areas of uncertainty in: 
weather conditions, friction coefficients (up to $10 \%$ margin of error), crush coefficient, roundingoff numbers (up to $+1 \mathrm{~km} / \mathrm{hr}$ ), hand calculation errors, driver behavior, or mechanism of crash. One group even mentioned the driver 'could have crashed due to dodging a wild animal' (case A). Teams held these uncertainties in mind when determining driver fault and determining initial car speeds. Students stated the vehicle speed as a range and compared this value against the posted speed limit, using their own line of reasoning and justification afterwards. Surprisingly, some teams stated that the driver is not at fault due to the uncertainty on the initial speeds calculated, or that the driver speed range fell on both sides of the speed limit. One group created a best case and worst case scenario by modifying the friction coefficients and given velocities.

\section{$\underline{\text { Values }}$}

Most of the student teams produced values that seemed reasonable, while a few teams' answers were off due to hand calculation errors. The following figures show the velocity values found by each team for each case and look at the percentage of student groups that chose to prosecute the driver for speeding. The dots represent the velocity found by a specific team. When there are two dots this means the data was given as a range. The speed limit is shown as the horizontal dotted line. The tables show the percentage of teams that prosecuted the driver in each case.

Note \#1: The two classes should not be compared for case A because they used different friction coefficients for the metal roof on asphalt which shifts the velocity answers substantially. Also, Case $C$ is different for the two classes, in one the cars stick together, in the other they slide separately. Note \#2: the data is sensitive to friction coefficients chosen and velocity angles chosen. 


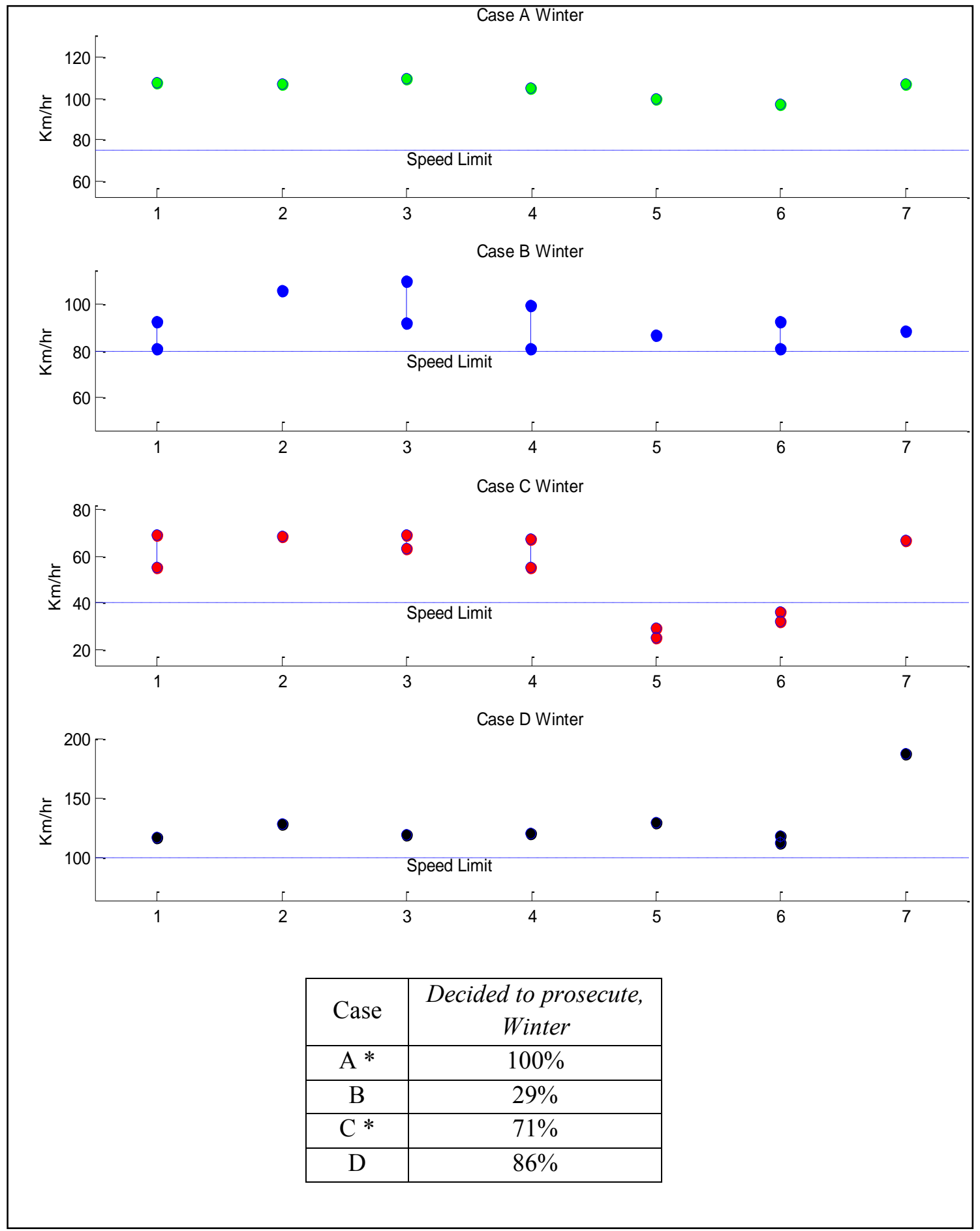

Figure 26. Winter Velocity Results. Each colored dot represents a velocity data point from one team, two dots stacked represents a range. 


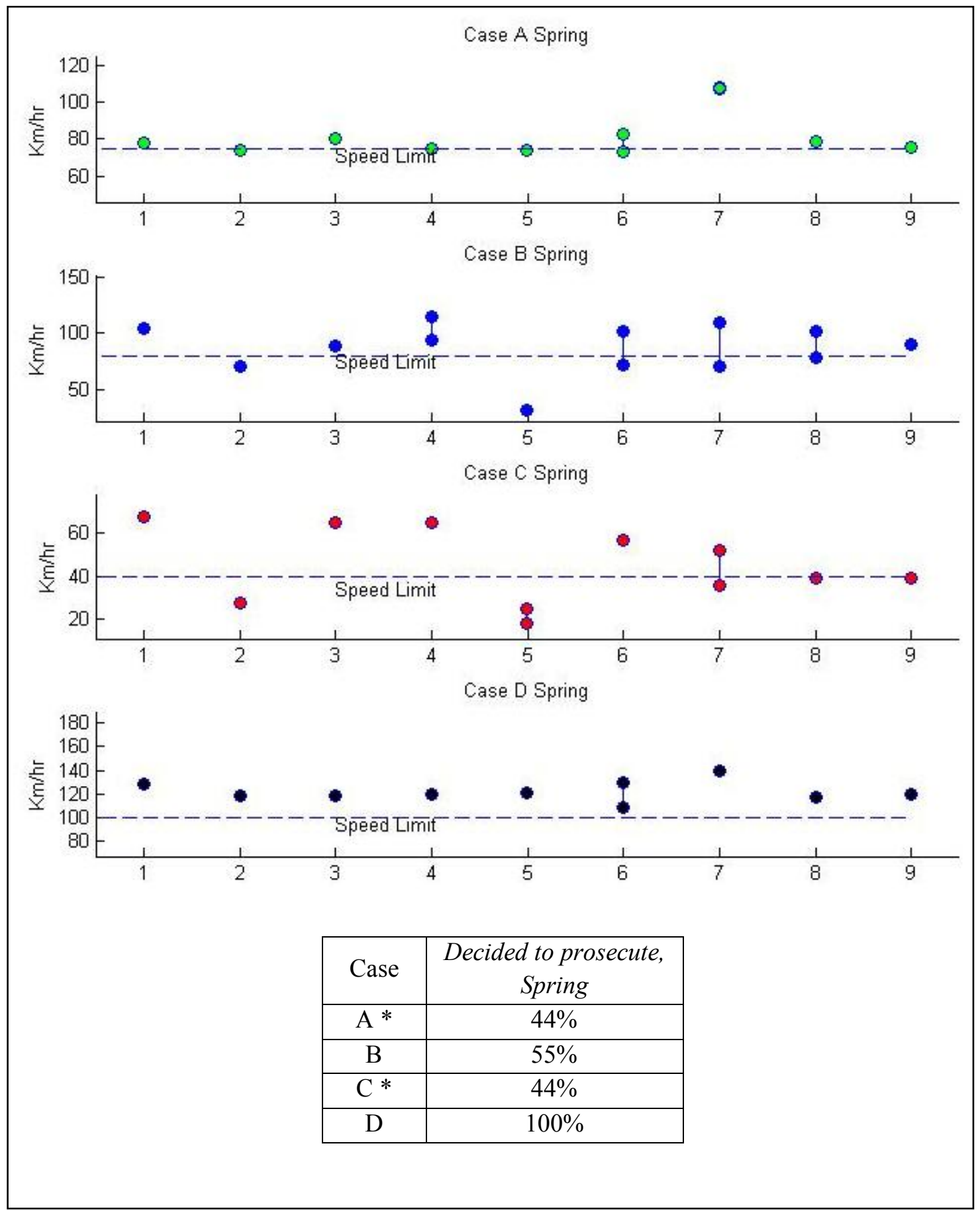

Figure 27. Spring velocity results. Each colored dot represents a velocity data point from one team, two dots stacked represents a range. 


\section{Excel Program}

Most of the winter teams used a clear, organized, and sometimes color-coded layout for their Excel programs. Some teams (3 out of 7) used one tab to create a general program while others had separate tabs to create a specific program for each accident case. A one-tab layout maintained the generalizability principle. Their Excel programs were structured in a way to deal with different types of accidents (one car or two cars, end up with cars sticking together or staying separate). Most spring groups (6 out of 9) used a one page/tab for their Excel program, which demonstrated that the intention was to use one tool to solve a variety of accident cases. The other groups (3 out of 9) used a separate tab for each accident case separately. As a note: the intention was for the students to generate a program which could handle any accident case and be generalizable to future situations.

\section{User Manual}

The user manuals listed steps to correctly use the program, as well give caution when inputting parameters like friction coefficients or staying in consistent units. The user manual also provided clear steps to follow to do accident reconstruction and caution to take at certain steps. Their user manuals also provided a list of parameters to collect at the accident scene (skid marks, skid angles, weather conditions, vehicle properties, etc.). Most of the winter user-manuals (5 out of 7) were clear and easy to understand, rated subjectively by the author. All spring teams (9 out of 9) did a good job in writing the user manual in a clear, instructive manner. 


\section{Survey}

A subjective survey prompted students for feedback and comments with the results shown below. A rating scale of $0=$ strongly disagree to $5=$ strongly agree was used.

Table 32. Survey Results for the VAR project

\begin{tabular}{|c|c|c|c|l|}
\hline $\begin{array}{l}\text { VAR } \\
\text { helped me } \\
\text { learn } \\
\text { dynamics }\end{array}$ & $\begin{array}{c}\text { VAR was } \\
\text { interesting } \\
\text { and } \\
\text { motivating }\end{array}$ & $\begin{array}{c}\text { VAR rate } \\
\text { importance } \\
(1 \text { is most, } \\
11 \text { is least })\end{array}$ & $\begin{array}{l}\text { It would be better to have us build } \\
\text { and experimentally test a scaled car } \\
\text { crash model than provide us with } \\
\text { the data for a car crash through the } \\
\text { police reports }\end{array}$ \\
\hline Winter & $3.85 / 5$ & $3.35 / 5$ & $6.31 / 11$ & $2.7 / 5$ \\
\hline Spring & $3.88 / 5$ & $3.59 / 5$ & $6.16 / 11$ & $3.0 / 5$ \\
\hline
\end{tabular}

Comments from different students:

- "The car crash project was set up incredibly. It made it more fun with the real scenario."

- "The car crash project seemed a bit too big in scale. It felt like there simply wasn't enough time to complete all types of cases in one excel file in the time given. Also, since there weren't any real life things to look at and the amount of simplification it got confusing after a while."

- “...I think it's an interesting application of Dynamics, so I don't think it should be cut out of the class completely. I think it would be better to do away with the Excel portion or to give better guidelines on how to approach making the program."

Students commented that the Excel program was tedious to create, and on top of that it was

difficult to make one sheet to handle all of the cases. While this project was difficult in nature, it is necessary for students to master these concepts in order to be competent in the course material.

From looking at these results, we can conclude overall ideas about this VAR project.

\section{Discussion}

Overall, students applied the relevant impact and energy principals in a realistic accident context.

As far as conceptual understanding, most groups understood the correct concepts and were able to apply them. Students did a good job of recognizing which parameters affected the velocity results and how uncertainties modified the results. 
Usually in MEAs, learning takes place when students create, revise, and document a model for the system. In this MEA, students re-constructed the accident and decided which parameters were important. I argue that self-assessment and model creation are the two of the most important values produced because they are subjective and not engineering equations; they show engineering judgment in an open ended problem.

The survey results were overall positive. From these survey results, the VAR project was worthwhile in helping students learn dynamics concepts, and in motivating them. With its overall positive response, the VAR project will continue in future quarters. I think that students were motivated by the realistic accidents, perhaps more so than a textbook problem.

\section{Improvements}

For future use, improvements will continue to be made to this activity. For instance, case D was taken out for the fall 2013 implementation because it was difficult to solve in their Excel program. As a replacement for case D, students were to create their own accident case. In order to help instructors check the velocity results and to develop future cases, the author wrote an Excel file. (Appendix I) 


\section{9: OVERALL ASSESSMENT OF MEAS}

We conclude with an overall section to summarize the effectiveness of the two MEAs. The first activity observed human leg motion dynamics, leading to an injury ranking procedure for a rehabilitation company. The second MEA investigated automobile crash dynamics, leading to an accident scene reconstruction procedure for police officers. The accident reconstruction and gait analysis activities both used the MEA approach to apply dynamics concepts, provide open-ended problem solving, motivate students, and practice professional communication. The modeleliciting activities (MEAs) followed six design principles seen below:

\section{Model Construction}

Students used their conceptual understanding to create a procedure for ranking or decision making of realistic quantities. Their ways of thinking were documented into their procedure, showing what parameters they found to be important and what assumptions they chose. For example, in the gait lab, some ranked the motions by injury type and corresponding loads, thus matching their engineering results to the real world. But other groups ranked their motions by forming a composite score value from different loads. This engineering model was used on an open-ended problem, where there was no right answer for the motion ranking lists or the driver speeding lists.

\section{Model Documentation}

Students documented their models in the gait and accident reconstruction activities through memos to the client, software files, and user manuals. In the memo, students responded to the client's request for work and explained their results in detail. The software file and user manual provided a procedure for the client to analyze future data. Students addressed the client directly and wrote in a professional tone which is usually different from traditional homework problems. 


\section{Generalizability}

Student teams created procedures which were applicable to a wide range of cases and could accept different input values. The gait procedure could accept different motions, body weights and lengths, while the vehicle accident procedure could accept different impact scenarios, vehicle properties, velocities, and friction coefficients. The Excel and Matlab files allowed users to solve future cases and problems. This allowed students to refine and extend their previous conceptual knowledge [16].

\section{Effective Prototype}

The concepts for these two MEAs are robust in terms of applicability to future academic and professional life. The vehicle accident activity teaches conservation of momentum and energy principles which can be used in future problems dealing with collision of bodies. The gait MEA teaches rigid body kinetics and kinematics which could be applied to dynamics of mechanisms. These concepts are important in dynamics and warrant the use of MEAs.

\section{Reality}

Both the vehicle accident and gait MEAs provided a realistic project for students, something they could potentially encounter when working on a real job. The clients were also realistic: the Sri Lankan Police department and a sports rehabilitation company. Students had to consider driving on the left side of the road in the vehicle MEA and considered ligaments and bones in the gait MEA. This realistic context adds to student motivation, which affects the time students are willing to spend on learning [23].

\section{$\underline{\text { Self-Assessment }}$}

Students checked their results to see whether they were reasonable. To check reasonable values, students could consult their past homework assignments or research literature values, or even 
connect to life experiences. For the vehicle accident MEA, students thought about realistic velocities from their driving experience. For example, driving a car over $100 \mathrm{~km} / \mathrm{hr}$ can be considered dangerous. For the gait MEA, groups could look up values in the literature for joint loads, such as running causes a force multiple of body weight.

They reported which sources of error were built into their model and what limitations existed due to the assumptions made (for example, '2D sagittal model'). The values and ranking order produced varied between groups based on the assumptions and chosen input parameters. The vehicle speeds in the accident reconstruction project were highly sensitive to friction coefficient values, and could change the outcome by $10 \%$. If students can successfully self-assess their results on this activity, then they are capable of applying this skill in future engineering careers or industry work.

\section{Other Aspects}

Engineering makes use of technology and powerful software tools, including Matlab and Excel. Advancing these software skills, even outside of MEAs, can help students build their engineering capabilities which can be applied in future work instances.

Working with teams was an important aspect of these projects. The instructor had students split into teams for each section of the project. Groups could share their data results and collaborate on the gait MEA. This promoted team skills, communication, and peer learning.

\section{Difficulty and Guidance}

The gait analysis was more complex and had more complaints from students than the accident reconstruction activity. Some steps to the gait activity were not directly stated by the instructor, which made it more difficult and not as smooth transitioning from task to task. For example, some tasks were to match the force plate to video data, and find the acceleration of the center of 
gravity of the limbs, which the instructor did not teach students at the beginning of the project. Midway through the activity, instructions and hints were provided when students reached certain stages. Besides the complexity of tasks, some students responded with irritation about writing the memorandum to the client.

\section{$\underline{\text { Future work }}$}

In the future, changes can be made to improve the MEAs. The vehicle accident activity has been run for five years as is at a relatively finished state. The gait MEA is a new activity, having been run for two sessions, and can be improved by the instructors to have better layout of instructions and tasks to give to students. The instructor can better explain the activity during class or assist during lab sessions, from having experienced multiple implementations. If the instructions and requirements are clearer to students then it will help them to work more efficiently. 


\section{0: OVERALL MEA CONCLUSION}

Overall these different activities benefit the class by producing outcomes in conceptual understanding, problem solving skills, and student attitudes. Concepts are learned through active engagement allowing students to document and reveal their thinking. Model-eliciting activities provide realistic contexts, more so than traditional methods, by offering a client and a real world problem. In addition, students work on their personal attitudes towards engineering analysis and communicating with a professional client.

In traditional instruction, students work on a single context homework problem or project and deliver results to the instructor. Traditional methods motivate students and develop their conceptual learning, but produce different outcomes than MEAS. Evidence for the beneficial outcomes of using MEA methods has been demonstrated by previous work in 2011, comparing performance between traditional and MEA methods classes.

A course survey asked the students to rate how well the course improved specific skills. These questions are related to the ABET criteria and can be used to help evaluate the course's overall effectiveness. See reference [12] for survey results. While many of the traditional course objectives for dynamics (e.g., "Ability to using engineering concepts to solve problems") are similar, there are many noticeable differences in student opinion between the two styles. One significant difference in the criteria was that MEA classes tended to produce higher outcomes on writing reports, working in teams, current issues, and knowledge of professional and ethical responsibility than traditional classes [12].

The instructor team has compared grades from MEA classes to traditional classes, to make sure scores were higher in the MEA sections to validate their use. During the fall of 2008, five of the eight dynamics sections implemented MEAs throughout the course, while the remaining sections 
followed a more traditional teaching style. The figure below shows the results of the exam and DCI scores with standard deviations.

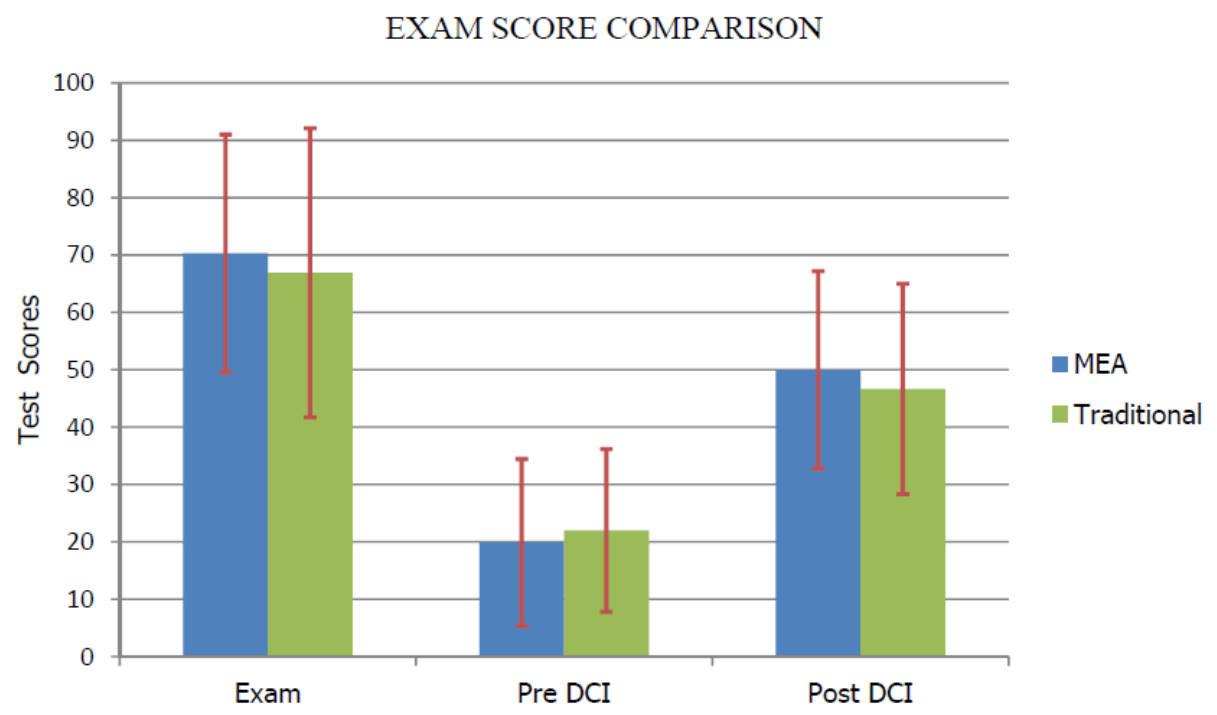

Figure 28. Exam Score Comparison between MEA and traditional classes.

The figure shows little difference between the exam scores for the sections. A t-test was performed with a p-value of 0.077 , which indicates no statistical difference between the sections (although the average final exam score was slightly higher for the MEA style classes). Students in the MEA based class showed significantly greater gains on the DCI test than traditional classes (29\% vs. $21 \%$ normalized gain) and slightly higher (although not statistically different) scores on the final exam [12]. The benefits of the MEA include motivation from addressing real world contexts, better professional attitudes, and increased conceptual gain as demonstrated above. The authors of previous MEA work [12] argue that engineering context and familiar applications should produce better long term retention when using the MEAs.

The significant normalized gain from these results is expected to hold for future MEAs. It is reasonable to expect similar results from using the relatively new gait analysis activity. Further refinement of the MEAs will help students to progress through the activity more efficiently and get more out of the activity, and potentially increase learning outcomes. 
The vehicle and gait MEAs challenged students on an extensive analysis project, more so than a homework assignment, and led them to think openly about a realistic problem. This setup produced varied results from student work as well as subjective feedback. They responded that the MEA helped to teach the material and motivate them, but also found the time commitment and writing portion to be demanding. Students worked hard on the MEAs and struggled through the tasks, while learning in teams, and eventually finished the activity with a more experienced viewpoint on the material. This experience and relevant skills can be transferred into real work situations and future engineering problems. These two MEAs will continue to be used in future classes and be further refined in their implementation to yield better student deliverables. Using MEAs in future course applications will expand the benefits to teaching students in additional conceptual content areas. 


\section{Active Learning at Cal Poly}

The four IBLAs and two MEAs in this thesis work have provided an engaging learning experience to build conceptual understanding and solve real life problems. The assessment results demonstrated learning outcomes and changes in student understanding throughout each quarter. Educational research will continue in the future, implementing active learning methods in undergraduate dynamics courses, to improve the teaching methods and student conceptual gains. The IBLAs and MEAs will be investigated to a finer level of detail to search for when students have the "aha" moment and seemingly grasp the concept in their own ways of thinking. The instructors designed the activities for student groups to explore dynamics concepts actively and repair their held misconceptions. Further research is being conducted using individual and group interviews on the IBLA activities in 2013.

It was an interesting experience from working with professors and seeing the work that is put into developing activities to produce meaningful learning. It was fun to get to know the students during their classes and be a teaching assistant. Often times the work load for the students was intense; there were many team projects and collaborative work, but the pain builds gain so to speak. Cal Poly really does a good job of "learn by doing", and challenging students to apply what they learn to semi-real world problems in hopes of shaping the engineers of tomorrow. 


\section{BIBLIOGRAPHY}

[1] Bonwell, C. 1991. The Enhanced Lecture: A Resource Book for Faculty. Cape Girardeau, MO: Southeast Missouri State University, Center for Teaching and Learning.

[2] Bonwell, C. 1996. Enhancing the lecture: Revitalizing a traditional format. In Using Active Learning in College Classes: A Range of Options for Faculty, edited by T. Sutherland and C. Bonwell. San Francisco, CA: Jossey-Bass Publishers.

[3] Bonwell, C., Eison, J. 1991. Active Learning: Creating Excitement in the Classroom. ERIC Digest.Washington DC. ERIC Clearinghouse on Higher Education.

[4] Bransford, J., A. Brown, and R. Cocking, How People Learn: Body, Mind, Experience and School. 2000, Commission on Behavioral and Social Science and Education, National Research Council: Washington, D.C.

[5] Cal Poly Catalog. 2011-2013. Mechanical Engineering Department.

[6] Chi, M.T. 2005. Commonsense conceptions of emergent processes: Why some misconceptions are robust. Journal of the Learning Sciences 14(2): 161-99.

[7] Evans, D. L., G. L. Gray, S. Krause, J. Martin, C. Midkiff, B. M. Notaros, M. Pavelich, D. Rancour, T. Reed-Rhoads, P. Steif, R. Streveler, and K. Wage. 2003. Progress on concept inventory assessment tools. In Proceedings of the ASEE/IEEE Frontiers in Education Conference. Boulder, CO.

[8] Georgette, J., Self, B.P., Widmann, J., Bohn, K., and Wang, E. (2013) Rolling, rolling, rolling: An inquiry-based learning activity in dynamics. Proceedings of the 2013 American Society for Engineering Education Pacific Southwest Annual Conference, Riverside, CA.

[9] Gray, G.L, Evans, D., Cornwell, P., Costanzo, F., and B. Self (2005), "The Dynamics Concept Inventory Assessment Test: A Progress Report," Proceedings of the 2005 American Society for Engineering Education Annual Conference.

[10] Hake, R. "Interactive-Engagement vs. Traditional Methods: A Six-Thousand-Student Survey of Mechanics Test Data for Introductory Physics Courses." American Journal of Physics, 1998: 64-74. 
[11] Hake, R. "Interactive-engagement methods in introductory mechanics courses.

Department of Physics, Indiana University, Bloomington, Indiana 47405.

[12] Heller, C, Self, B.P. Accident Reconstruction: A Model-Eliciting Activity in Dynamics. ASEE Conference 2011. PSW American Society for Engineering Education Zone IV Conference.

[13] Hestenes,D. and Wells, M. and Swackhamer, G. (1992). Force Concept Inventory. The Physics Teacher Vol 30. p 141-151.

[14] Laws, P., D. Sokoloff, and R. Thorton. "Promoting Active Learning Using the results of physics education research." Uniserve Science News, 1993. 13

[15] Lesh, R., \& Zawojewski, J.S. (2007). Problem solving and modeling. In F. Lester (Ed.), Second handbook of research on mathematics teaching and learning (pp. 763-804). Charlotte, NC: Information Age Publishing.

[16] Lesh, R., Hoover, M., Hole, B., Kelly, A., Post, T., (2000) Principles for Developing Thought-Revealing Activities for Students and Teachers. In A. Kelly, R. Lesh (Eds.), Research Design in Mathematics and Science Education. (pp. 591-646). Lawrence Erlbaum Associates, Mahwah, New Jersey.

[17] Lightman, A. and P. Sadler, Teacher Predictions Versus Actual Student Gains. The Physics Teacher, 1993. 31: p. 162.

[18] Meriam, J.L. and Kraige, L.G. (2006) Engineering Mechanics, Volume 2, Dynamics, 6th Edition, John Wiley \& Son r.)

[19] Minstrell, J.A. 1989 Teaching science for understanding. Pp. 130-131 in Toward the Thinking Curriculum: Current Cognitive Research, L.B.Resnick and L.E.Klopfer, eds. Alexandria, VA: Association for Supervision and Curriculum Development.

[20] Picture copyright from McGraw Hill

[21] Prince, M. (2004) Does Active Learning Work: A Review of the Literature. Journal of Engineering Education, pp 223-231.

[22] Prince, M. and Vigeant, M. (2006) Using Inquiry-Based Activities to Promote Understanding of Critical Engineering Concepts. Proceedings, American Society for Engineering Education Annual Conference and Exposition. 
[23] Prince, M. J. and Felder, R. M. (2006), Inductive Teaching and Learning Methods:

Definitions, Comparisons, and Research Bases. Journal of Engineering Education, 95: 123-138.

[24] Rittle-Johnson, B. 2006. Promoting transfer: Effects of self-explanation

and direct instruction. Child Development 77 (1): 1-15.

[25] Rittle-Johnson, B., R. S. Siegler, and M. W. Alibali. 2001. Developing conceptual understanding and procedural skill in mathematics: An iterative process. Journal of Educational Psychology 93 (2): 346-62.

[26] Ruhl, K., C. Hughes, and P. Schloss. "Using the Pause Procedure to Enhance Lecture Recall." Teacher Education and Special Education, 1989: 14-18.

[27] Self, B.P. NSF grant proposal. Using Inquiry-Based Activities to Repair Student Misconceptions in Engineering Dynamics.

[28] Self, B. Birdsong, C, Rossman, E. “A New Spin on Teaching 3D Kinematics and Gyroscopic Motion". American Society for Engineering Education, 2008.

[29] Self, B.P., Dr. James M Widmann Dr. Michael J. Prince (Bucknell University), and Jeffrey Georgette. Inquiry-Based Learning Activities in Dynamics. ASEE 2013 National Conference, Atlanta.

[30] Self, B.P., Widmann, J., Prince, M.J., and Georgette, J. (2013) Inquiry-based learning activities in dynamics. American Society for Engineering Education Annual Conference and Exposition.

[31] Self, B. P., Miller R. L, Kean, A., Moore, T. J., T. Ogletree and F. Schreiber, Important student misconceptions in mechanics and thermal science: identification using modeleliciting activities , $38^{\text {th }}$ Annual Frontiers in Education Conference, Saratoga Springs, NY, October, 2008.

[32] Streveler, R., Litzinger, T., Miller,R., Steif,P. "Learning Conceptual Knowledge in the Engineering Sciences: Overview and Future Research Directions." Journal of Engineering Education, 2008: 279-294.

[33] Thacker, B., et al., Comparing problem solving performance of physics students in inquirybased and traditional introductory physics courses. American Journal of Physics, 1994. 62(7): p.627. 
[34] The New Mechanics Conference held August 6-7, 1992 at Tufts University, was attended by Pat Cooney, Dewey Dykstra, David Hammer, Priscilla Laws. Suzanne Lea, Lillian McDermott, Robert Morse, Hans Pfister, Edward Redish, David Sokoloff and Ronald Thornton.

[35] Zawojewski, J.S., Diefes-Dux, H.A., Bowman, K.J., Models and Modeling in Engineering Education. Designing Experiences for All Students. Sense Publishers, 2008.

[36] NSF grant: 717595: Collaborative research: improving Engineering students' Learning Strategies through Models and Modeling.

[37] Winter, D.A. Biomechanics and Motor Control of Human Movement, Second edition. John Wiley \& Sons, Inc., Toronto, 1990. 


\section{APPENDICES}

\section{APPENDIX A: Mass-Pulley worksheets}

\section{When handling the pulleys:}

-Release the masses in each case from at least 25 inches above the ground.

-Be careful with the rope because it can twist and tangle easily.

-Wind up the rope in a spiral when you are done with the activity.

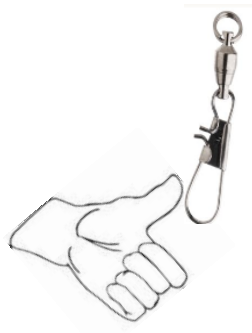

-Attach masses at its yarn loop and use metal clasp attached to the main rope.

-To switch the masses around, open the clasps with your thumb (see side picture)

\section{Case A:}

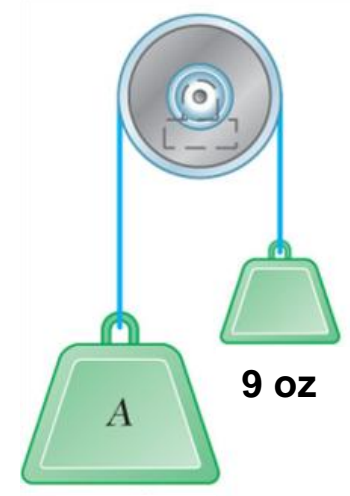

$10 \mathrm{oz}$

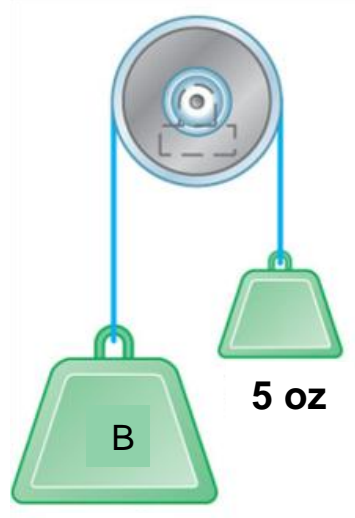

$6 \mathrm{oz}$

1. Consider the masses A and B with weight as shown. What do you predict about the accelerations of the masses if they are released from rest? Indicate the \# of votes on your team of the four give possibilities below.

Mass A will accelerate downwards faster than mass B Mass B will accelerate downwards faster than mass A Mass A and B will accelerate downwards at the same rate Neither Mass A or B will accelerate downwards

2. What did you observe when performing the experiment?

2. Please explain the results of your experiments using dynamics principles. 


\section{Case B:}
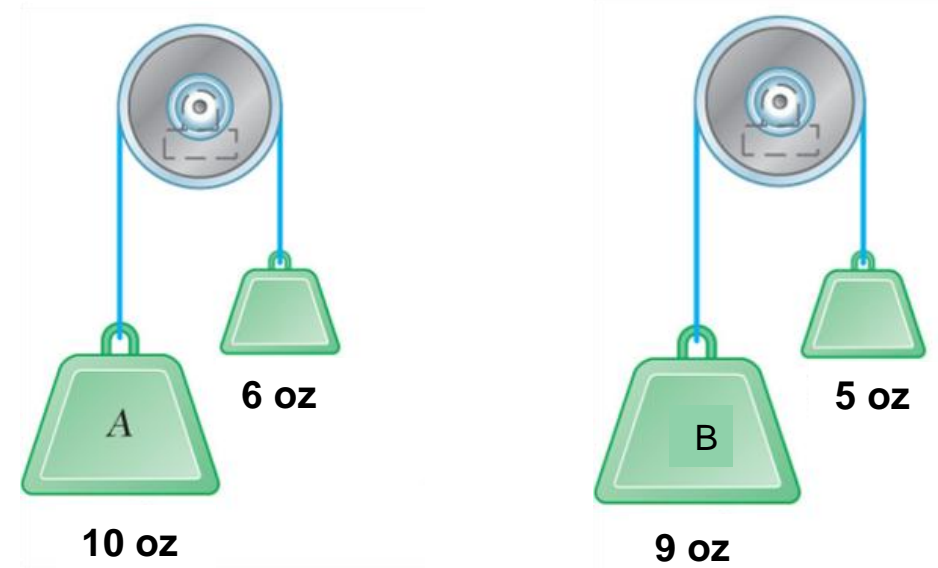

1. Consider the masses A and B with weight as shown. What do you predict about the accelerations of the masses if they are released from rest? Indicate the \# of votes on your team of the four give possibilities below.

Mass A will accelerate downwards faster than mass B Mass B will accelerate downwards faster than mass A Mass A and B will accelerate downwards at the same rate Neither Mass A or B will accelerate downwards

2. What did you observe when performing the experiment?

3. Please explain the results of your experiments using dynamics principles. 


\section{Case C:}

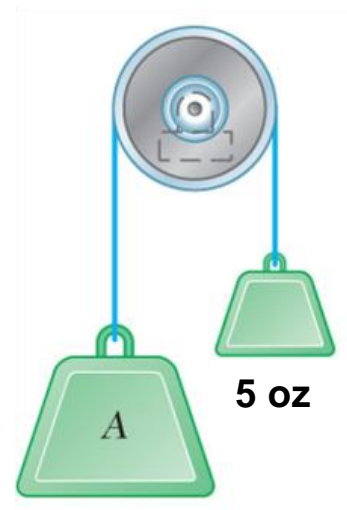

$10 \mathrm{oz}$

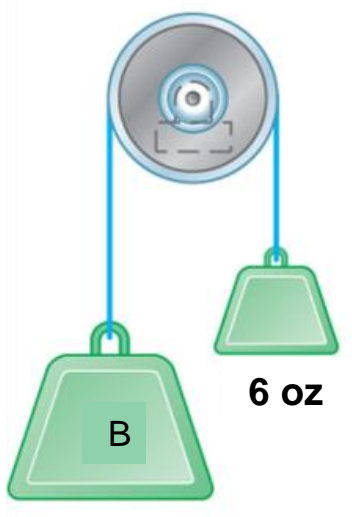

9 oz

1. Consider the masses A and B with weight as shown. What do you predict about the accelerations of the masses if they are released from rest? Indicate the \# of votes on your team of the four give possibilities below.

Mass A will accelerate downwards faster than mass B Mass B will accelerate downwards faster than mass A Mass A and B will accelerate downwards at the same rate Neither Mass A or B will accelerate downwards

2. What did you observe when performing the experiment?

3. Please explain the results of your experiments using dynamics principles. 


\section{APPENDIX B: Spool Worksheet}

Follow the directions in order - wait for the assistants to have you enter answers using PollEverywhere before you discuss any of the questions or do any of the activities below.

1. Answer the PollEverywhere questions about the horizontal pull.

2. Discuss the question: if you pull on the string gently in the horizontal direction as shown, which way will the spool move?

$$
\text { (Indicate \# of votes): Right ___ Left }
$$

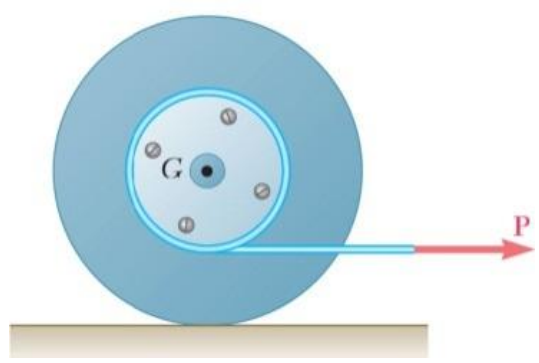
Won't Move

3. Discuss the question: if you pull on the string gently in the horizontal direction as shown, in which direction will the friction force act?

(Indicate \# of votes): Right ___ Left ___ Impossible to tell

4. Pull gently on the string in the configuration shown. Which way does the spool move? Which direction is the friction force? What is the value of the friction force?

5. Now pull on the string a bit harder so that it isn't rolling without slip. Which way do you think the friction force acts? It is probably in the same direction as above, but now it will be equal to what value? (if you have time after doing this, start drawing out your FBD and KD)

Don't turn the page over until instructed to do so. 
6. Answer the Polleverywhere questions about the vertical pull.

7. Discuss the question: if you pull on the string gently in the vertical direction as shown, which way will the spool move?

(Indicate \# of votes): Right ___ Left ___ Won't Move

8. Discuss the question: if you pull on the string gently in the vertical direction as shown, in which direction will the friction force act?

(Indicate \# of votes): Right Left Impossible to tell

9. Now pull gently on the string vertically. Which way does the spool go? Which direction is the friction force? What is the value of the friction force? If you have time, draw out your FBD and KD.

10. Try varying the angle of your pull, and how hard you pull on the string. When is the friction force equal to $\mu_{\mathrm{s}} \mathrm{N}$ ? $\mu_{\mathrm{k}} \mathrm{N}$ ? Explain your answers. 


\section{APPENDIX C: Rolling Objects Worksheets}

\section{$\underline{\text { Rolling Objects Activity }-\mathrm{S} 13}$}

$\underline{\text { Setup }}$

Create an incline with the ramp with a height of several inches using a book or steps. At the bottom of the ramp place a backpack or clothing to cushion the objects.
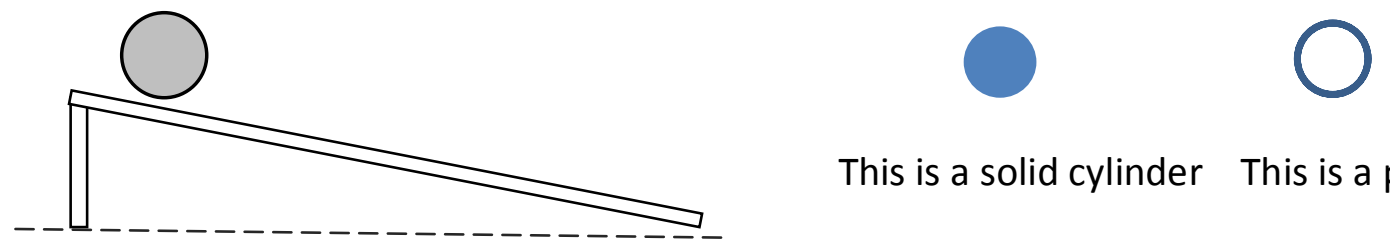

This is a solid cylinder This is a pipe

For each exercise below, read the description and write your prediction on the anonymous prediction sheet before testing. It is okay for your answers to be wrong, this sheet is not graded! Please do not change them after you roll the objects. After recording your prediction, place the rolling objects close to the top of the ramp, side by side and held by the starting gate. Have the starting gates as vertical as possible. Flip the starting gate handle as quickly as possible to create a 'fair' start. When the objects roll to the bottom of the ramp catch them or use a cushion to stop them so they are not damaged by bouncing on the stone ground. Run the following scenarios and respond to the prompts. Perform each exercise multiple times, with different objects on each side of the ramp. How much of a difference do think it takes for there to be a clear-cut winner (e.g., 1 inch? 5 inches?).

\section{$\underline{\text { Exercises }}$}

1) Take the big metal solid cylinder and the black metal pipe. (Same radius and mass). Write your prediction on the separate prediction sheet. Roll the two, and state the post-race result below. How do you explain the race result using principles of Dynamics?

2) Next, take the small metal solid cylinder and the big metal solid cylinder. (Different radius and mass). Write your prediction on the separate prediction sheet. Roll the two, and state your results below. How does mass influence rolling behavior?

3) Take the small metal solid cylinder and black metal pipe. (Different shape, mass, and radius). Write your prediction on the separate prediction sheet. Roll the two, and state the post-race result below. How do the cylinders compare to each other? 
4) Take the small PVC pipe and big PVC pipe and grey metal pipe. (Same shape, different radius and mass). Write your prediction on the separate prediction sheet. Roll them, and state the post-race result below. What is the rolling behavior of pipes?

5) Which has bigger Total Kinetic Energy when it reaches the bottom, the big metal solid cylinder or black metal pipe? (same mass and radius)

6) Which has bigger Total Kinetic Energy when it reaches the bottom, the small metal solid cylinder, the wood solid cylinder or the big metal solid cylinder? (different mass, radius)

7) All solid cylinders regardless of radius and mass arrive at the same time True False

8) All thin walled pipes regardless of radius and mass arrive at the same time True False

9) Which will arrive first, a thick walled pipe or a thin walled pipe regardless of radius and mass? Thick walled all Thin-walled 

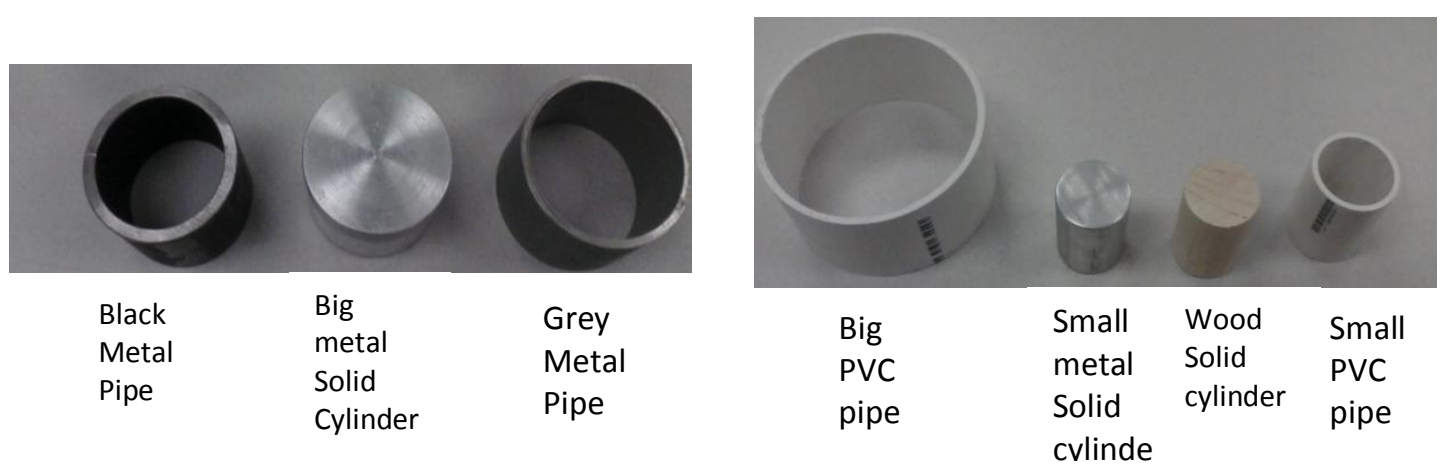

1) Take the big metal solid cylinder and the black metal pipe. (Same radius and mass). Which will get to the bottom first?

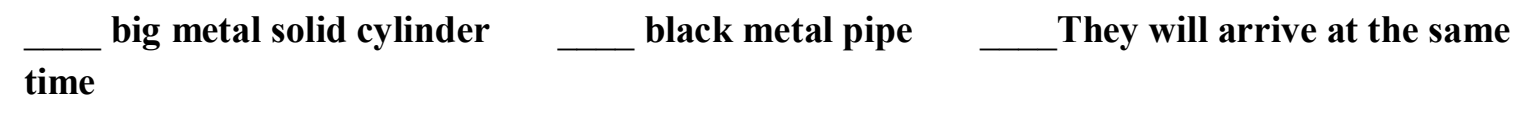

2) Next, take the small metal solid cylinder and the big metal solid cylinder. (Different radius and mass, same shape). Which will get to the bottom first?

small metal solid cylinder

big metal solid cylinder

They will arrive at the same time.

3) Take the small metal solid cylinder and black metal pipe. (Different shape, mass, and radius). Which will get to the bottom first?

\section{time} small metal solid cylinder

black metal pipe

They will arrive at the same

Take the small PVC pipe and big PVC pipe and grey metal pipe. (Same shape, different radius and mass). Which do you predict will get to the bottom first, second, third place? Indicate with a "1", "2" and " 3 ". If you think some will tie, give them the same number. small PVC pipe big PVC pipe 


\section{APPENDIX D: DCI Question for Rolling Objects}

The two objects in the figure at the right are released from rest at the position shown and roll without slipping down identical hills. Both objects have the same mass $m$ and same outer radius. Object $A$ is a thin hoop whose mass is concentrated in its outer edge. Object $B$ is a uniform

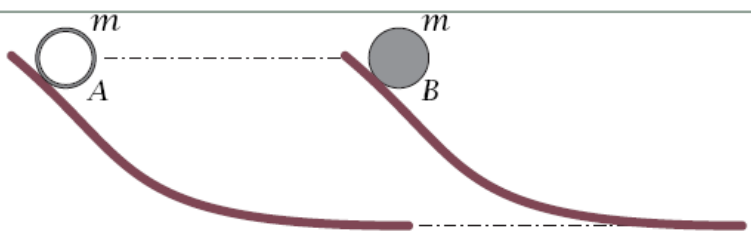
solid cylinder. Neglecting air resistance, how do the speeds of the two objects compare when they reach the bottom of their respective hills?

(a) $A$ and $B$ will have the same speed.

(b) The speed of $A$ will be greater than that of $B$.

(c) The speed of $B$ will be greater than that of $A$.

(d) Knowledge of the friction forces is required to answer the question.

(e) Knowledge of the shape of the cross-section of the thin hoop is required to answer the question. 


\section{APPENDIX E: Gyroscope Worksheet}

Gyroscope Mini-Lab (you will turn this in at the end) SPRING 2013

Recall : -- I $\quad \vec{M}=\dot{\vec{\varphi}} \times I \dot{\vec{\psi}}$ and $\dot{\psi}$ are about spin axis, $\dot{\phi}$ denotes precession

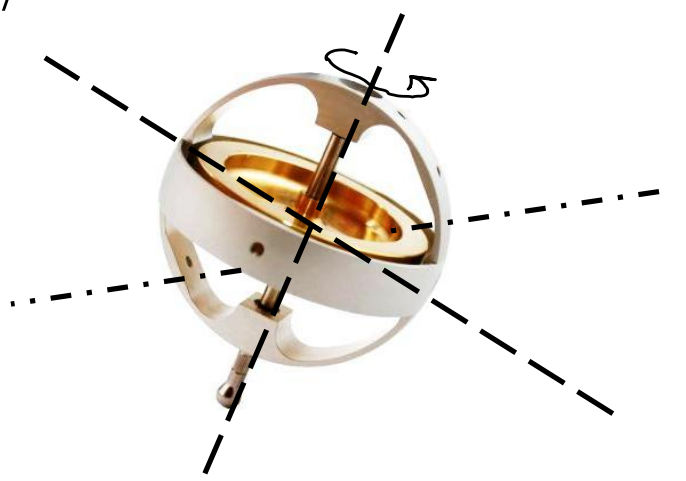

\section{Precision Gyroscope (Please do not drop these; they are very expensive)}

Please record your predictions on a separate, anonymous sheet.

\section{STATION 1}

Make your prediction under Station 1(a) on the Prediction Sheet

To spin-up the gyro, connect the electric motor to the top part on the gyro, and turn on the switch on the motor box. Looking at it from the top (where you attach the motor), the rotor will rotate counterclockwise. Make sure to remove the motor from the gyro after spin-up.

A short rod is attached to the outer ring; hold the rod in the air at an angle above horizontal and release.

What causes the rod to precess?

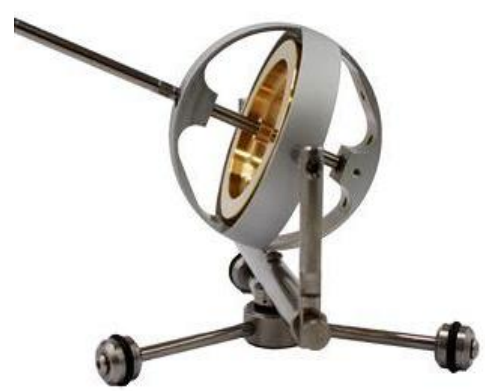

Watch the gyro precess. Push up or down gently on rod. How does the motion change as the rod is moved up and down? Are you surprised by the force necessary to move the rod up or down?

After the experiment, Sketch a figure with vectors to help explain. Describe in words what is happening with regards to the angular momentum.

Read the next description and Make your prediction under Station 1(b) on the Prediction Sheet.

Next, attach the weight to the end of the rod. How does the added weight change the speed of precession? 


\section{STATION 2}

Read the description below for the next activity, before doing the activity, and make your prediction under Station 2(a) on the Prediction Sheet.

Let the rotor point at some oblique angle (not vertical or horizontal). While holding the base, slowly slide the system around on the table. (Prediction 2a).

After that, slowly lift a base leg off the table surface, no more than 1 inch high. Do this for each leg. (No prediction for lifting a base leg off the table). The orientation of the rotor should point the same direction even if the base moves since there is no moment applied to the rotor (due to the gimbals).

Why does the spin axis remain at the same orientation? If the rotor has constant spin speed, how might a spinning rotor be used to help orient a satellite?

Make a prediction under Station 2(b) on the prediction sheet about the gyro tilt direction from pushing on the right side of the gimbal.

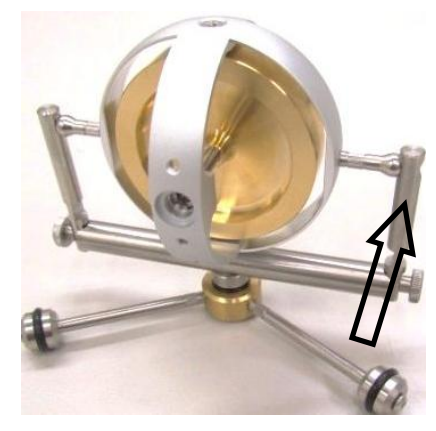

With the rotor spinning in the vertical plane as shown, gently push on one side of the gimbal.

Explain what happens using a figure and the gyroscopic equation.

\section{STATION 3}

\section{Gyroscopic Bicycle Wheel}

Read the description below for the next activity, and before doing the activity, make your prediction under Station 3(a) on the Prediction Sheet

Have one person on the team hold the wheel as shown, and a second person push down on the wheel to get it spinning (spin direction on figure). Rotate your body to the right, (clockwise from above), over $360^{\circ}$.

After doing the activity, what do you feel from the handles as you turn your body right? What do you have to do to the wheel to make this motion happen?

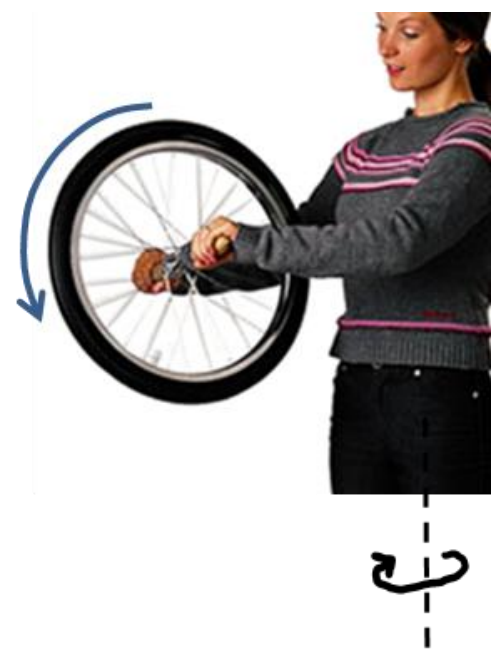

Read the description below, and before actually doing the activity, make a prediction under Station 3(b) on the Prediction Sheet. 
Hold one of the wheel handles with one hand, so the other handle pointing straight away from you. As you look out from your body/arm, spin the wheel $\mathrm{CW}$. Then rotate your arm and body to the right.

After running the activity, show how and why this happens using a sketch and appropriate equations.

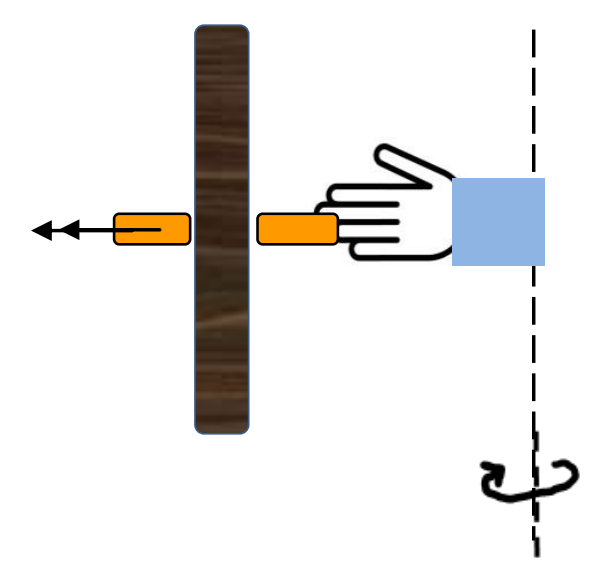

Read the description below, and before actually doing the activity, make a prediction under Station 3(c) on the Prediction Sheet.

You will need to share one of the rotating platforms with the other bicycle wheel groups, so some of you should start with part $d$.

c) You will need to get the wheel spinning fairly fast and do the demo right after it starts spinning. Hold the wheel in front of you like shown in the picture. Tilt the bike wheel to the left about 30 degrees (if you did this through a full 90 degrees it would be horizontal).

After doing the exercise, What happens? Move it back. Describe what and why this happens.

d) Read the description below, and before actually doing the activity, make a prediction under Station 3(d) on the Prediction Sheet.

Lastly, spin the wheel in a vertical plane as fast as you can with the string attached onto a side handle (like the figure at right). Then hold onto the string and watch what happens to the wheel gyroscope.

After running the activity, What happens and why? Explain your answer using a sketch of the vectors and the simplified gyroscopic equation
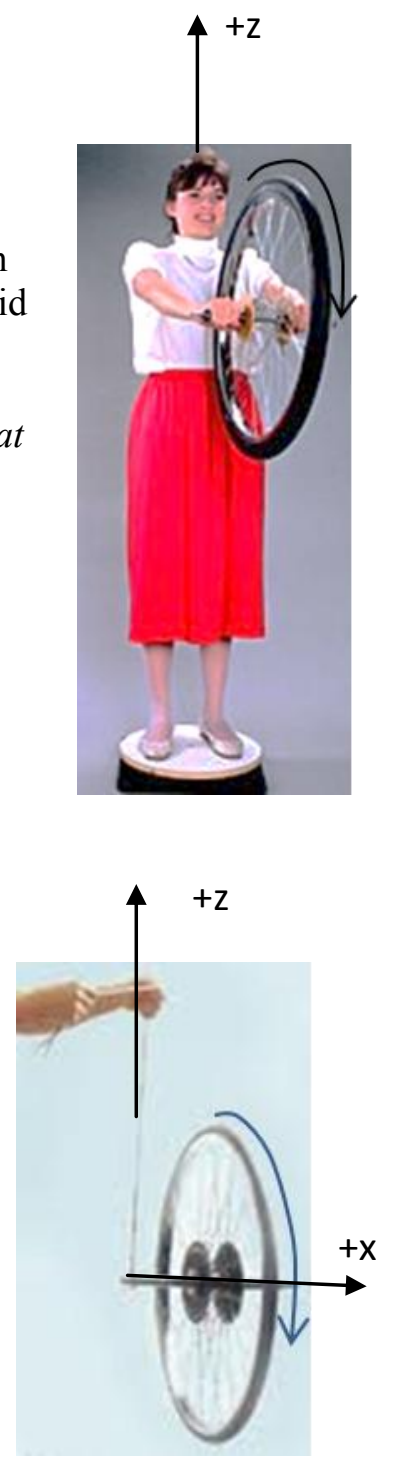


\section{APPENDIX F: Gait Analysis Memo}

Dear IntDyn Engineers,

We would like to propose a project to your engineering team. We are a rehabilitation company whom caters to athletes and active members of society, providing motion bracing systems and recommendations. Though we have a product line of motion bracing systems and a physical therapy network, we seek the help of your engineering team

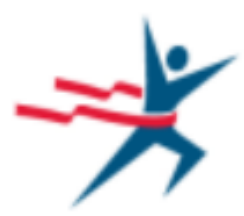
EnMotion to provide analysis of various motion activities.

- We need specific guidelines which we can give to our network of physical therapists and to our patients. More specifically, we need guidelines on when and how to limit motion activities. Our patients have sustained injuries in their knees and legs so that protective measures must be offered to them.

- We would like you to analyze different activities such as walking, walking using a cane, crouching, sitting in a chair, lunging, and picking up a box.

- Also, the assessment requires a way to rank or determine if such activities are dangerous to the patients since we do not want our patients to injure themselves further or impair their quality of life. This ranking will also be used to allow patients to "move up" to more demanding tasks as their injuries heal. Please quantify and qualify measures of "dangerous" actions and when motions can be deemed "safe", which will allow us to rank the activities listed and other activities which our patients may wish to undergo. Please provide us with this ranking mechanism, along with an explanation of how you established it. To collect experimental data, tools are provided at the kinesiology lab: a force plate to measure ground reaction forces, and a kinematic camera to record leg motion.

- Please submit your MatLab code for analyzing leg motion which can be used for any given motion data.

- Please respond with a memo detailing your findings and guidelines which we can provide to physical therapists).

- Additionally, we would like you to provide us with a short paragraph that we can give to our patients that will give general guidelines on activities that they should avoid.

See attached for relevant information. We look forward to your work.Sincerely,

John Brightestone

John Brightestone

Vice President. EnMotion Co.

Los Angeles, CA 
EnMotion was founded in 1993 by Mr. Brightestone to fill the need of sports rehabilitation to professional athletes, both professional and collegiate, and to common people engaging in physical activity. The company has grown substantially in the last decade due to outstanding customer service to patients and the adoption of new technology. EnMotion engineers and sports analysts strive to provide for the needs of customers and society, allowing them to work hard and play hard.

\section{Engineering Information}

\section{Measuring Tools}

Force Plate Sensor - measure foot-ground reaction forces vs time Kinematic Camera - measure position of limbs vs image frame

\section{Mass Properties:}

See Dempster handout

\section{Items of analysis:}

Forces $\mathrm{Fx}, \mathrm{Fy}, \mathrm{Fz}$, Moments Mx,My,Mz from force plate

Positions $\mathrm{x}, \mathrm{y}, \mathrm{z}$ of: foot, toe, ankle, hip from kinematic video camera

\section{Gait Analysis Memo \#2}

Dear IntDyn Engineers,

We look forward to your turn-ins next week and your full response to our first memo. Please let us know your rankings of the six different activities (dangerous to safe), how you reached those decisions, and a process for us to rank future activities (plus all the other things we mentioned in our previous correspondence to you).

In your Appendix, please supply us with support materials, including your hand calculations for your analysis, an explanation of your analysis process so we can repeat it in our own experiments, and plots versus time of: internal knee forces and moments, shin acceleration, anterior-posterior ground reaction forces, and vertical ground reaction forces. Provide these for your teams' full analysis of the two different activities. Also, include all Matlab files and data files for your chosen code (just one) in your zip file so that we can run your full simulation.

Sincerely,

John Brightestone

John Brightestone

Vice President. EnMotion Co.

Los Angeles, CA 


\section{APPENDIX G: Matlab Code for Gait Analysis MEA}

The author wrote a Matlab file to assist the professors with checking students' gait lab results. The code took force plate and kinematic data as inputs and analyzed dynamics of the leg, as discussed before. Ultimately, the code yielded knee forces in the axial and shear directions, the out-of-plane moment of the knee, and shin CG accelerations. These values can be compared to student results for each athletic motion to validate their ranking of motions.

The process is as follows: the code reads the force plate data and video kinematic data, as well as user-inputted subject parameters. The force plate data and video data are manipulated by trimming to the same length to match up properly, and smoothed/interpolated as necessary to filter the noisy data. From the video data, the marker position yields the shin CG position, velocity, and acceleration, found for both translation and rotation senses. Summing the forces in the horizontal and vertical axes and summing moments about the shin CG leads to the forces and moments at the knee joint - the joint of importance in this project. The knee forces are then transformed to a relative frame which moves with the shin to give axial-shear components, which are more relevant in the physical analysis compared to $x-y$ components. Plotting these parameters (limb/joint positions) allows visual aid to check that the results are reasonable.

Some notes on the nuances of the data analysis are as follows. The accuracy of the output values depends upon the chosen inputs (which can vary from team to team). Such parameters as pixelmeter conversion, tolerance on smoothing splines, the placement of physical markers, the digitizing placement of digital markers, etc., change the numerical value of the output. For this reason, the code results should be compared to students' work with a degree of tolerance to account for the variance in user settings.

This code will be improved upon in the future with updates to the MEA; perhaps some sections of the lab will be cut and new sections will be added, so the code will adapt with time. The limb animation portion of the Matlab code was written by Michael Hoover. 
$\%$ Matlab Code for gait lab MEA analysis Updated $11 / 20 / 2013$ written by Jeff Georgette for MS Thesis , help by Michael Hoover, Alex Baucom

\% ME 326 Intermediate Dynamics Cal Poly San Luis Obispo Model Eliciting Activity Gait Lab

$\%$ This code analyzes the joint forces and accelerations of the

foot,ankle,knee, and hip of a person in motion Force data is captured by a force plate, data is read in. Video data is captures by a video camera, data is read in. Data is cut down to length, interpolated to expand, and smoothed out. Force data is combined with acceleration(position) data in the sum of the forces in the $x, y$ directions and the moment in the $\mathrm{z}$ direction about the center of mass of the shin. 'Shin' is combination of shin and foot.

Units are Newtons,radians,meters,seconds.

$\%$ Get ready! Here we go

$\%$

clear; clc;

close all;

$\%$ clear all

$\%$ Input Persons Measurements

$\%$ weight = input('Please enter the subject"s weight in pounds ');

weight $=134 ; \quad \%$ weight in ( pounds) mass $=$ weight $/ 2.2046$;

$\%$ mass in $(\mathrm{kg})$ body_weight $=$ mass $* 9.81 ; \%$ (Newtons)

body_weight $=587 ; \%$ Newtons, name Juan walk

mass $=$ body_weight $/ 9.81 ; \% \mathrm{~kg}$

$\%$ mass properties of inertia taken from Dempster.

$\mathrm{m} \_$shin $=$mass $* 0610 ; \%$ Mass of the Shank $(\mathrm{kg})$

$\%$ Length shin is calculated by markers, later in code.

$\mathrm{g}=9.81 ; \quad \%$ gravity constant $\left(\mathrm{m} / \mathrm{s}^{\wedge} 2\right)$

\%Input Data files and paramaters for import

force_data_file $=$ 'Juan Walk.xlsx'; \% Force plate data

video_data_file='Juan Walkxypts.csv'; \% Video frame data

vid_rate $=1 / 30 ; \%$ video capture rate

$\mathrm{fp}$ rate $=1 / 300 ; \%$ force plate capture rate

conversion $=1 / 220 ; \%$ video pixel to meter conversion

\section{$\% \%$ Import force plate data}

stepsize $=\mathrm{fp} \_$rate; $\%$ force plate capture rate $300 \mathrm{~Hz}$

start_flag $=0 ; \%$ flags to signal start of motion

end_flag $=0 ; \%$ flag to signal end of motion

time_mark_start $=0$; time_mark_end $=0 ; \%$ store the time and value of start

val_mark_start $=0$; val_mark_end $=0 ; \%$ store the time and value of end

threshold_val $=10 ; \%$ threshold of automatic detection of

$\%$ motion timeframe, (Newtons)

intt $=10 ; \%$ quanity of data points to add to interpolate force video $\%$ data

$\%$ Clip off headers in excel. Import force plate,[time,Fx,Fy,Fz,Mx,My,Mz]

fpdata $=$ xlsread(force_data_file);

time_array $=\operatorname{fpdata}(:, \overline{1})$; 


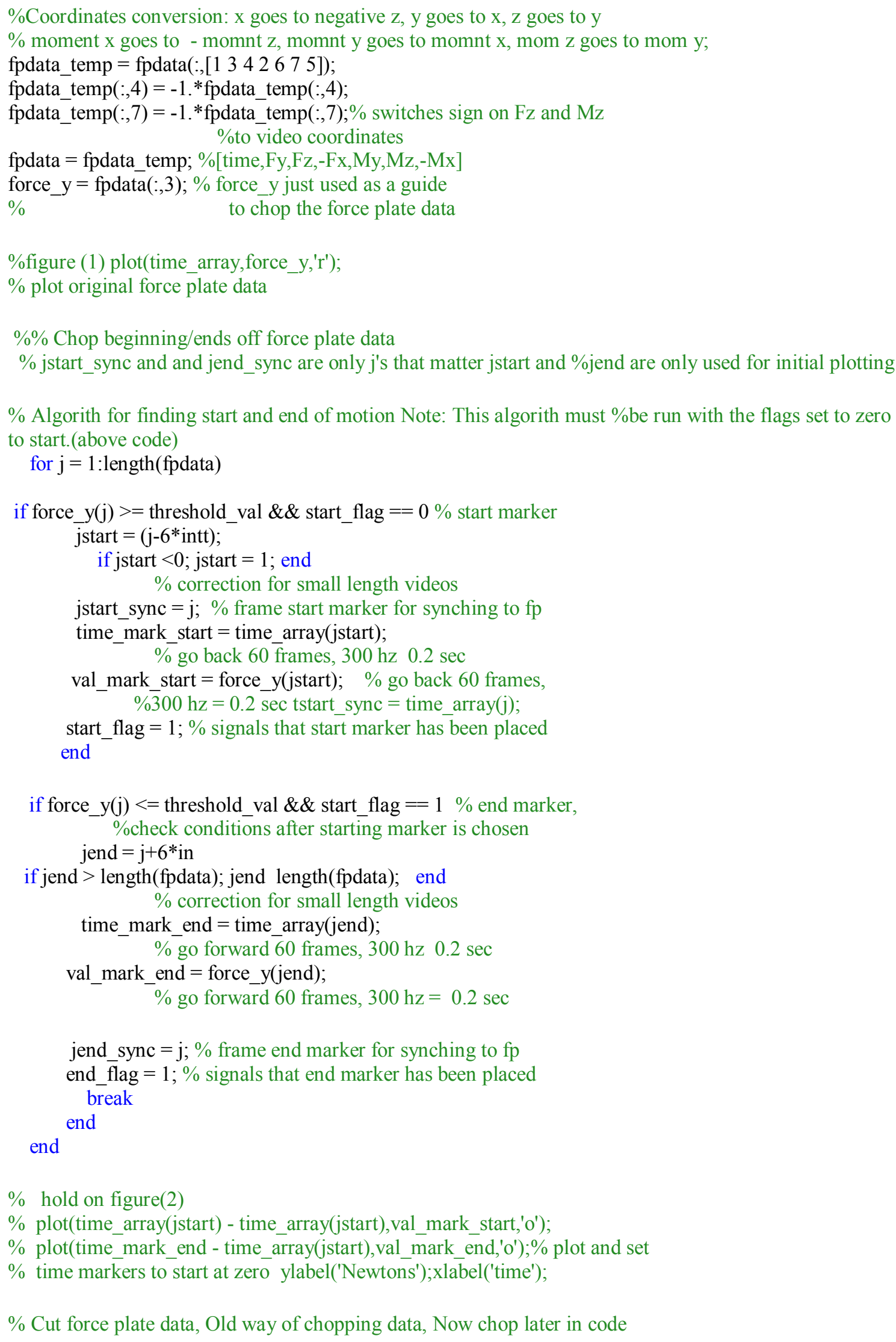

if force_y(j) $<=$ threshold_val $\& \&$ start_flag $==1 \%$ end marker, $\%$ check conditions after starting marker is chosen jend $=\mathrm{j}+6{ }^{*}$ in

if jend $>$ length(fpdata); jend length(fpdata); end $\%$ correction for small length videos

time_mark_end = time_array(jend); $\%$ go forward 60 frames, $300 \mathrm{hz} 0.2 \mathrm{sec}$

val_mark_end $=$ force_y(jend);

$\%$ go forward 60 frames, $300 \mathrm{hz}=0.2 \mathrm{sec}$

jend_sync $=\mathrm{j} ; \%$ frame end marker for synching to fp end_flag $=1 ; \%$ signals that end marker has been placed break end

end

$\%$ hold on figure(2)

$\%$ plot(time_array(jstart) - time_array(jstart),val_mark_start,'o');

$\%$ plot(time_mark_end - time_array(jstart),val_mark_end,'o');\% plot and set

$\%$ time markers to start at zero ylabel('Newtons');xlabel('time');

$\%$ Cut force plate data, Old way of chopping data, Now chop later in code 
$\%$ when synching code figure $(2)$ force_y_chop $=$ force_y(jstart:jend $) ;$
$\%$ created trimmed force_y data force_plate_chop $=$
$\%$ (fpdata(jstart_sync:jend_sync,: $)) ; \%$ created trimmed force_y data
$\%$ time_array_chop $=$ time_array(jstart:jend)-time_array(jstart);
$\%$ create trimmed timed data and set start time to zero
$\%$ plot(time_array_chop,force_y_chop); $\%$ plot chopped force plate data
$\%$ forceplatelength $=$ time_array_chop $($ end $) ; \%$ seconds

\section{$\% \%$ Import video position data}

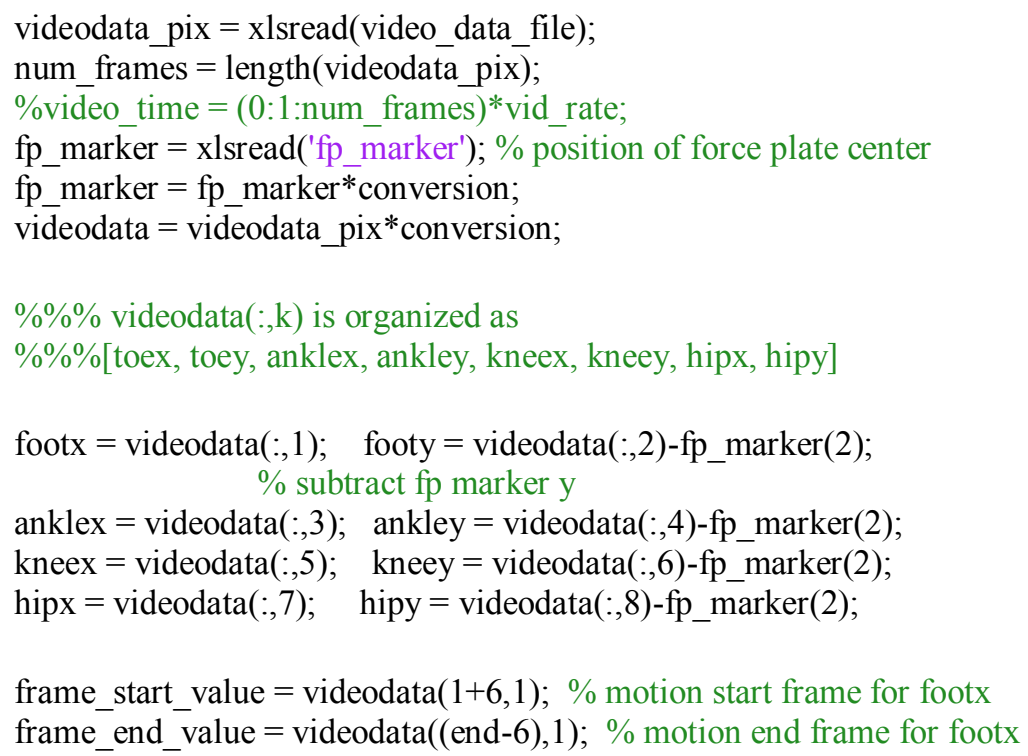

\%initialiaze array for interpolated data(row,column)

videodata_int $=$ zeros $($ intt*size $($ videodata, 1$)$,size $($ videodata, 2$))$;

\%Interpolate data by 'intt' points $(\mathrm{intt}=10)$

for $\mathrm{k}=1$ : size(videodata, 2 )

videodata_int(:,k) = interp(videodata(:,k),intt); \% interpr(array,\# points) 
end

$\%$ Sets frame start and frame stop for interpolated and thus smoothed data.

num_frames_int $=$ num_frames*intt;

frame_start_flag $=0$; frame_end_flag $=0 ; \%$ flag prevents over writintg

\%values, by chance, later.

for $\mathrm{i}=1$ :num_frames_int $\%$ grabs new frame numbers

$\% \quad$ for the start and stop of motion

if abs(videodata_int(i,1)-frame_start_value) $<0.0001 \& \&$ frame_start_flag $=0$

frame_start_int $=\mathrm{i}$; frame_start_flag $=1$;

elseif abs(videodata_int(i,1)-frame_end_value $)<0.0001 \& \&$ frame_end_flag $==0$

$\%$ tweak these values to it grabs the right frame

frame_end_int $=\mathrm{i}$; frame_end_flag $=1$;

else

end

end

length_int $=($ frame_end_int - frame_start_int +1$) ; \%$ length of 'motion'

$\%$ hold on for $\mathrm{i}=2: 2 \%$ plot interpolated data

$\%$ plot(videodata_int(:,2*i-1),videodata_int(:,2*i),'s','MarkerFaceColor','g','MarkerSize',4);

$\%$ end

$\% \%$ Smooth position kinematic data

$\%$ 'videodata_int' is interpolated data

$\% \quad$ while 'position_sm' is interpolated and smoothed data

position_sm $=$ zeros(size(videodata_int));

for $\mathrm{i}=1$ :size(position_sm,2) \% loop over columns

$\mathrm{a}=1$ :size(videodata_int, 1 ); \% horizontal axis data

$\%$ (choose time or frames, basically plotting your $\mathrm{x}$ axis)

$\mathrm{b}=$ videodata_int(:,i); $\%$ data column to be smoothed

$\% \operatorname{plot}\left(\mathrm{a}, \mathrm{b}, \mathrm{o}^{\prime}\right) ; \%$ plot raw position data

tol $=0.001 ; \%$ tolerance on spline sharp/smoothness

$\%$ Play around with tolerance: small tolerance $=$ sharpness, big

$\%$ tolerance $=$ smoothness. See how it affects your graph Set

$\%$ Tolerance values anywhere from .00001 , to 20 , to 10,000

$\%$ depending on scale of data.

$\% \mathrm{~m}=1 ; \%$ linear spline, $\% \mathrm{~m}=3 ; \%$ quintic spline

$\mathrm{m}=2 ; \%$ cubic spline, default

$\% "$ "[object,values] $=$ spaps(x_data,y_data,tolerance $)$

$\%$ spline maker"

[blah_object,values] = spaps $(\mathrm{a}, \mathrm{b}, \mathrm{tol})$;

$\%$ 'values' are smoothed data, 'blah_object' is not used here.

position_sm(:,i) $=($ values' $) ; \%$ builds smoothed data matrix

end

$\% \% \%$ PLot of joint markers which have been interpolated $/$ smoothed $\% \%$

$\%$ hold on $\%$ plots points in motion for $\mathrm{i}=1: 4 \%$ plot interporlated

$\%$ data 


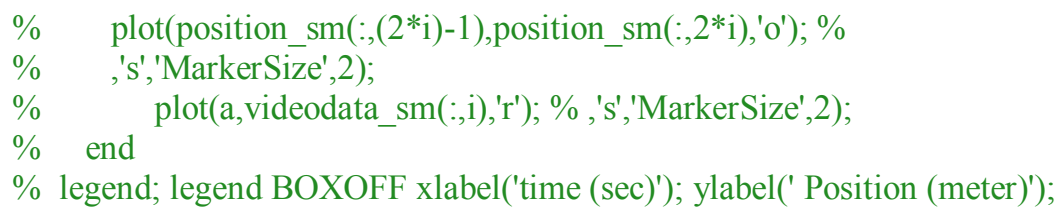




\section{$\%(\operatorname{shin}+$ foot $)$ about cg}

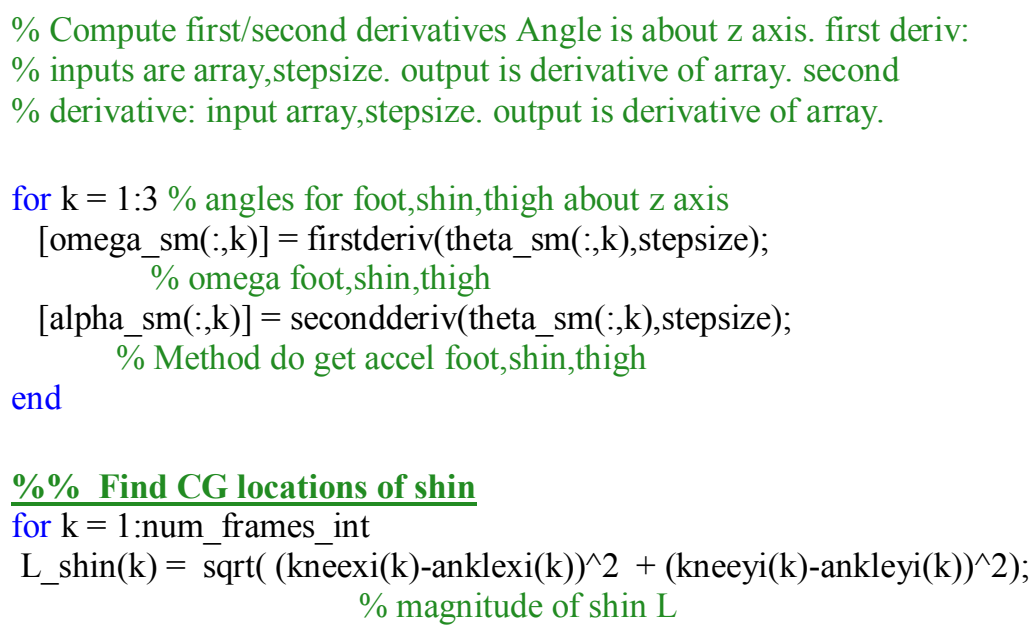

$\% \%$ Acceleration of CG SHIN 


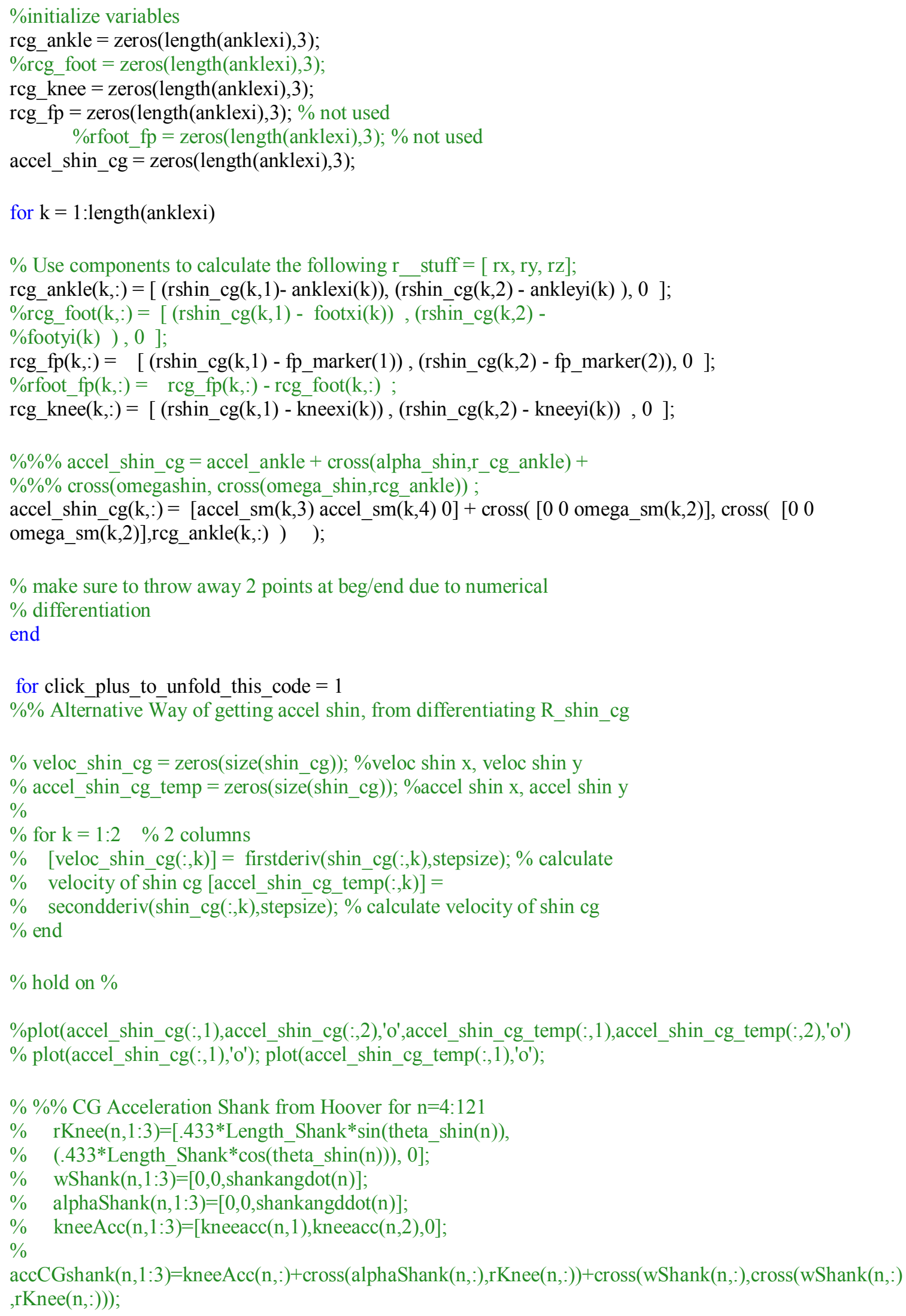


$\%$ end

end $\%$ old code here

\section{$\% \%$ Forces}

$\% \% \%$ To line up force plate data with video data, use start of motion on

$\% \% \%$ fp plate, then go the quantity frame time of video,

$\%$ and call this point the end of the force plate data

$\% \% \% \%$ Synchronizing force plate to video data $\% \% \%$

$\mathrm{c}=$ jstart_sync;

if (jstart_sync+length_int-1) <= length(fpdata(:,1))

$\%$ correction check to avoid errors

$\mathrm{d}=$ (jstart_sync+length_int-1);\% this is the normal case

else

$\mathrm{d}=$ length(fpdata(:,1));

$\%$ creates $d$ ( end force plate frame) based upon video length

disp(' warning: force plate end-sync-frame corrected ');

end

force plate_chop $=$ fpdata(c:d,: );

$\%$ chop force plate data to appropriate length

$\% \% \%$ Add 2 rows of zeros before and after force_plate_chop, to line up

$\% \% \%$ with acceleration array:

$\mathrm{zz}=$ zeros(2,size(force_plate_chop,2));

$\mathrm{C}=\{\mathrm{zz} ;$ force_plate_chop; $\mathrm{zz}\}$;

$\%$ adds 2 zero above rows, and 2 below rows

force_plate_chop $=\operatorname{cell} 2 \mathrm{mat}(\mathrm{C}) ; \%$ 'cell2mat'combines matrices

$\% \% \%$ Force_plate_chop $=[$ time,Fx,Fy,Fz,Mx,My,Mz $]$ but chopped to video

$\% \%$ length.

fx_plate $=$ force_plate_chop(:,2); \% from force plate

fy_plate $=$ force_plate_chop(:,3);

$\%$ fz_foot $=$ force_plate_chop $(:, 4)$;

$\% \% \%$ initialize more variables at chopped lengths

mom_foot $=[$ zeros(length(fy_plate),1), zeros(length(fy_plate),1), force_plate_chop(:,7)];

$\%$ taken from $\mathrm{fp}$ in video coordinates,

mom_knee $=$ zeros(size $\left(\operatorname{mom} \_\right.$foot $\left.)\right) ; \%$ matrix

mom_shin $=$ zeros $(\operatorname{size}($ mom_foot $))$;

$\mathrm{fx} \_\mathrm{knee}=\operatorname{zeros}\left(\operatorname{size}\left(\mathrm{fx} \_\right.\right.$plate $\left.)\right)$

$\%$ initialize variables so they are right orientation

fy_knee $=$ zeros(size(fy_plate) $)$;

faxial_knee $=$ zeros $($ size $($ fy_plate $))$;

fshear_knee $=$ zeros(size $($ fy_plate $)$ );

$\% \% \%$ 'mag_force_knee 1,2 ' used to compare $\mathrm{fx} / \mathrm{fy}$ and faxial/fshear. \% (i.e. vector resultant magnitudes should be the exact same).

$\%$ mag_force_knee $1=$ zeros $\left(\operatorname{size}\left(f x \_f o o t\right)\right)$; mag_force_knee2 =

$\% \quad z e r o s\left(\operatorname{size}\left(f x \_f o o t\right)\right.$ ); 


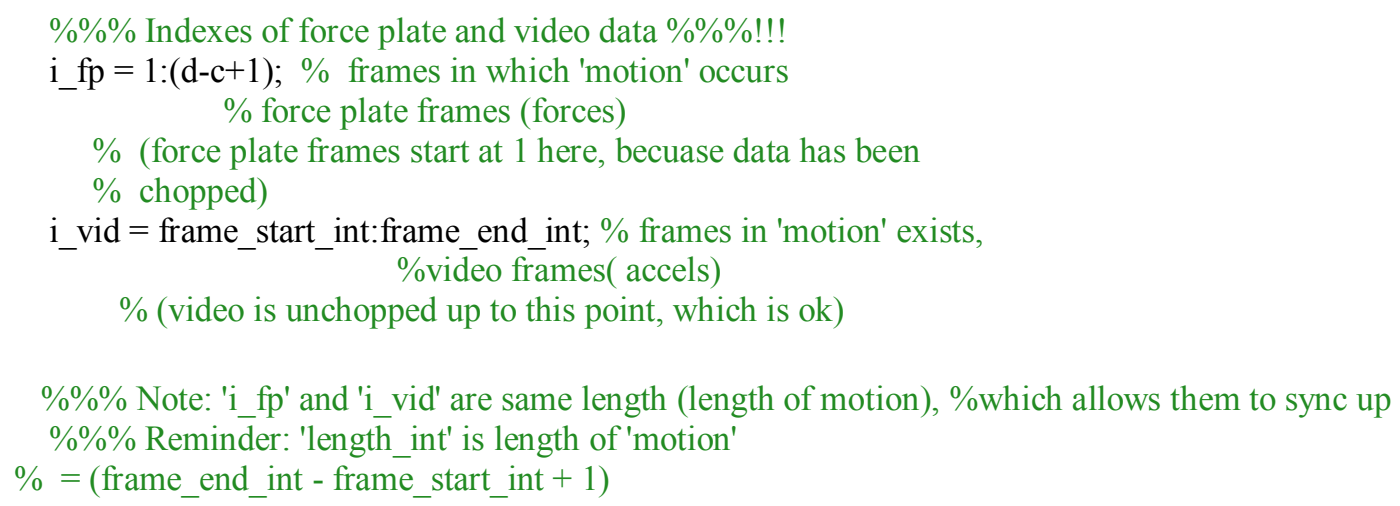

end

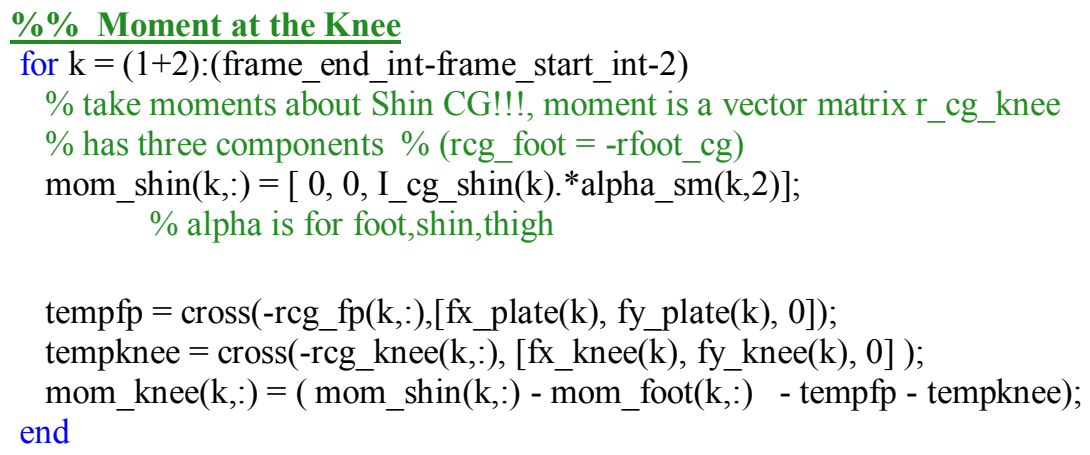


$\% \%$ PLOTS

time $\operatorname{plott}=$ force plate $\operatorname{chop}(3:$ length $(\mathrm{i}$ fp $)-2,1)$;

$\%$ time array used for plotting purposes. has correct indexes.

figure $(2) \%$ plot shin cg accelerations x y

hold on

plot(time plott,accel_shin_cg(3:length(i_fp)-2,1),'-s','MarkerFaceColor','b','MarkerSize',4);

plot(time_plott,accel_shin_cg(3:length(i_fp)-2,2),'-s','MarkerFaceColor','g','MarkerSize',4);

legend('Accel shin $\operatorname{cg}^{-} x^{\prime}, '$ Accel shin $\left.\operatorname{cg} y^{\prime}\right)$;

legend $\mathrm{BOXOFF}$

xlabel('time (sec)');

ylabel(' Acceleration $\left.\mathrm{m} / \mathrm{s}^{\wedge} 2^{\prime}\right)$;

figure(3); \% Plot axial and shear forces at knee

hold on

plot(time_plott,faxial_knee(3:length(i_fp)-2)/body_weight,'-s','MarkerFaceColor','g','MarkerSize',4);

plot(time_plott,fshear_knee(3:length(i_fp)-2)/body_weight,'-s','MarkerFaceColor','b','MarkerSize',4);

legend('Force Axial Knee/ body weight','Force Shear Knee/body weight');

legend BOXOFF

xlabel('time (sec)');

ylabel(' Force/BW (Newton)');

figure(4) \% Plot moment at knee

plot(time_plott,mom_knee(3:length(i_fp)-2,3),'-s','MarkerFaceColor','g','MarkerSize',4);

legend('Moment Knee in opposite direction');

legend $\mathrm{BOXOFF}$

xlabel('time (sec)');

ylabel(' Moment (Newton-Meter)');

tempaa $=\max ($ abs $($ faxial_knee $))$;

tempbb $=\max (\operatorname{abs}($ fshear_knee $))$;

fprintf('Max force axial knee is \%3.3f (\%f* body weight) $\mathrm{N} \backslash \mathrm{n}$ ',tempaa,tempaa/body weight ); fprintf('Max force shear knee \%3.3f (\%f* body weight N) $\backslash n$ ',tempbb,tempbb/body_weight);

fprintf( 'Max moment knee is \%3.3f N-m $\backslash n \backslash n ', \max \left(\operatorname{abs}\left(m o m \_k n e e(: 3)\right)\right)$ );

\section{$\% \%$ Line Visualization for Limbs}

for $\mathrm{pp}=1$

$\%$ for $\mathrm{k}=1$ :num_frames_int $\%$ plot coordinate $\mathrm{k}$ of ankle to knee

$\%$

$\% \mathrm{xy}=[\mathrm{hipxi}(\mathrm{k})$ hipyi(k); \% position data for gplot

$\% \quad$ kneexi(k) kneeyi(k); anklexi(k) ankleyi(k); footxi(k) footyi(k)];

$\%$

$\% \quad \mathrm{~A}=\left[\begin{array}{lllll}0 & 1 & 0 & 0 & 0\end{array} \%\right.$ Oonnectivity Matrix

$\% \quad 1010$

$\% \quad 0101$;

$\left.\% \quad \begin{array}{llllll}\% & 0 & 1 & 0\end{array}\right]$

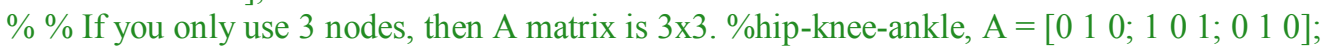

$\%$ hold on plot(hipxi,hipyi,'s'); \% plot hip node as square

$\%$ plot(kneexi,ankleyi,'d'); \% plots knee as diamond

$\%$ plot(anklexi,ankleyi,'o'); \% plot ankle as circle

$\% \operatorname{gplot}(\mathrm{A}, \mathrm{xy}) ; \%$ connects markers with a line axis equal hold on

$\% \mathrm{M}(\mathrm{j})=$ getframe; end

$\% \%$ movie(M)

end 
$\% \%$ Animation by Michael Hoover

$\%$ Uncomment below code to run animation.

$\mathrm{R}=$ frame start int; \% start from video chopped-frame

$\mathrm{S}=$ frame end $\overline{\mathrm{int}} ; \%$ end at video chopped-frame

Animation(footxi(R:S), footyi(R:S), anklexi(R:S), ankleyi(R:S), kneexi(R:S),kneeyi(R:S), hipxi(R:S),hipyi(R:

S),fshear_knee,faxial_knee,0) 


\section{APPENDIX H: Vehicle Accident Reconstruction MEA memo}

\section{Background:}

Sri Lanka Police Department - Traffic Police

from www.police.lk/index.php/traffic-police accessed 1/30/2013

Traffic Police Headquarters was established in 1953 and it assists the Inspector General of Police in taking decisions on traffic policies and thereafter it helps to implement them and closely monitor implementation. Policing of road traffic in Sri Lanka has become a major task for the Police. Implementation and Enforcement of regulations and Laws comes through powers vested on the Police by the Motor Traffic Act of 1951.The necessity to form a separate unit to control traffic within the city was recognized in 1950 by the Colombo Metropolitan Police. Due to the increase in volume of road traffic in the island the Traffic Headquarters was inaugurated in 1953 to cover the entire island.

1. Every station presently maintains a traffic branch. Officers entrusted with this specific duty are identified by the white coloured top part of their peak caps and the white belt with cross belt they wear. These officers have undergone extensive training in vehicle examining, traffic accident investigations and court procedures

2. Traffic wardens employed by the controlling bodies in the cities and towns assist the Police to a certain degree of parking of vehicles within town limits.

3. With the increase of the numbers of vehicles on the highways causing continuous traffic congestions in the cities especially during the peak hours, the demands on the Police to meet with the situation for smooth running of traffic has a corresponding increase.

\section{Main Functions}

1. Enforce Traffic Laws, prevent violations of traffic regulations and prosecution of offenders

2. Investigate into accidents.

3. Control traffic on highways.

4. Provide pilot duties for VIPP

5. Assist the public in various social events and functions where motor traffic is involved.

\section{Excerpt from Introduction to Forensic Engineering by Randal Noon}

\section{Vehicle Accident Reconstruction}

The reconstruction of vehicle accidents can be a very difficult task. In most cases, the engineer will be asked to reconstruct the events of an accident long after the accident has occurred.

Sometimes, the actual accident scene will be prohibitively far away from the engineer or will have changed by the time he is given the reconstruction assignment.

Relying upon the often conflicting information provided by witnesses or the accident participants can be confusing and misleading. Often, the witnesses will report their own conclusions and opinions instead of objective observations; sometimes the accident participants will knowingly or unknowingly lie about the events. Under these circumstances, obtaining factual information with which to work can be trying. 
However, the engineer will usually have the following reasonably objective information available to him at the outset:

1. The police accident report. The police report will contain the usual basic Identification information of the accident participants. It will also note the position of the vehicles after the accident as found by the police, the location of skid marks, the point of impact, the general layout of the scene, weather and conditions data, and the general travel pattern of the vehicles before the accident.

2. Photographs of the damaged vehicles. This is usually available from the insurance companies involved or their adjuster agents. They are used in evaluating insurance compensation to the accident participants.

The engineer may be asked to provide information or opinions about many aspects of the case, including some that are not related to the mechanical collision events. However, the engineer is nearly always asked to determine the initial velocities of the vehicles.

As discussed in the attached memo, your team will be given two different accident scenarios to use to set up your initial program/spreadsheet. Then, two new scenarios will be posted. Your final turn-in with an analysis of all four accidents (and details on how you used the Excel spreadsheet to solve them), other deliverables, and your cover memo must be uploaded to Polylearn. 


\title{
Memorandum
}

\author{
To: $\quad$ Forensic Engineering Team \\ From: $\quad$ H. M. B. G. Kotakadeniya, Senior Deputy Inspector General of Police, Sri \\ Lanka Police Service \\ RE: $\quad$ Traffic Accident Reconstruction Protocol \\ Prionity: [Urgent]
}

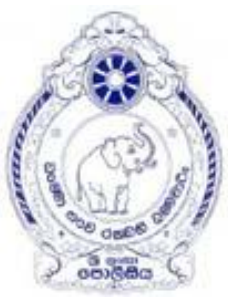

Since 2003 your country has been providing assistance toward development and economic stabilization here in Sri Lanka. Relations have gotten even closer with the invaluable help we received following the devastating tsunami in 2004. As a result, we have been able to become an important figure in the fight against terror in South-Central Asia.

As you may already know, the Sri Lanka Police Service has recently launched a new programme to update and modernize the service we provide to the public. One key area for improvement is in the Traffic Police Division. This division was established in 1953 to assist in making decisions on traffic policies and implementing them. Every currently existing station maintains a traffic branch, but the growing number of drivers on the island and our intention to build new stations demand that we immediately improve our accident investigation protocol. I am charging you with the task of compiling a new computer tool for accident investigation that can be used in this division. At the moment the main focus of this task is to develop an Excel program for determining if a driver has violated the speed limit.

My officers will provide you a set of two abridged incident reports that are characteristic of typical accidents that we regularly investigate - please refer to our online site for these reports. We are compiling an additional two reports that will also be provided to you at a later date. For legal reasons, sections of the reports have been omitted and the names of those involved have been replaced. In each report you will find a general description of the accident followed by more detailed information pertaining to possibly relevant parameters in the accident.

In order to determine whether your skills and approach are suitable to this task, please review and provide your opinion about two accidents (the accident reports are attached). Specifically we would like you tell us your estimate of the drivers speed prior to the accident. Please forward this information with any supporting hand calculations to us by Monday February $4^{\text {th }}$.

Next, we request that you provide the following to our office by Monday, February 11th: (1) an Excel program that we can use to estimate driver speeds for almost any accident scenario, (2) a User's Manual that teaches the basic principles of accident reconstruction to our officers and provides instructions on how to use the program, (3) instructions on what parameters the officers should collect at the scene, (4) your detailed analysis on how you used your program to solve each of the four accidents, (5) hand calculations that verify your program results for the four accident scenarios, and (6) a cover memo that discusses your conclusions for each of the accident scenarios and if you think we should prosecute the drivers. This memo should also include a discussion on if you think we should prosecute the drivers in each of the scenarios, especially given the uncertainty in some of the values used in your analysis (e.g., our friction coefficients can vary by $10 \%$ in many cases).

I am certain that your team will exceed our expectations.

H. Kotakadeniya

H. M. B. G. Kotakadeniya 


\section{APPENDIX I: Excel file for VAR MEA}

In order for instructors to verify student solutions for future accident cases, the author has provided an Excel file to perform vehicle accident analysis. This Excel file takes accident scene parameters as inputs (known velocities, friction coefficients, road grade, known velocity angles, bumper coefficients, skid distances) and outputs initial vehicle velocities. From these results the vehicle's velocity can be compared to the local speed limit to advise whether prosecution due to speeding is admissible.

The program is organized by different areas. First the user inputs the vehicle and scene parameters. Next, the program has the user start from the end of the crash and work towards the beginning. After inputting all of the parameters, the program automatically produces the vehicle velocities before the crash occurred. A series of question boxes are presented in which the user selects a choice in order to run the program. The input prompts are shown below:

- Did any vehicle's bumper get crushed?

- Yes car1, yes car 2, yes both cars crush, no bumper crush

- Did any cars skid after the collision?

- Yes car 1, yes car 2, yes both cars together, yes both cars separate, no skid

- What kind of collision occurred?

○ Oblique Collision and Stick, Head on Collision and stick, Head on Collision and no stick, No Collision

- Did any cars flip over?

- Yes car 1, yes car 2, no car flipped

- Did Any Cars skid before the collision occurred?

- Yes car 1, yes car 2, both skid, no skid

- Now solve for Initial Velocities

- Car 1, Car 2

The Work-Energy equation was used during instances of bumper crushing, tire and roof skidding, and car flipping. Changes in potential and kinetic energy were related to non-conservative work 
done. Also, changes in potential energy were due to cars ascending and descending on an inclined road. The kinetic energy at each phase of the crash yielded the vehicle's velocity. The conservation of momentum equation was used during head-on and oblique collisions between two cars. Using conservation of momentum, vehicle velocities of pre and post collision were found using known velocities and vehicle masses.

Due to solving difficulty one situation was omitted (two cars colliding obliquely when entering and exiting at different angles, and not sticking together).

The sign convention for this program is as follows. For an incline road or road grade, positive direction is up the ramp and negative direction is down the ramp. For oblique crashes, the angle convention is (looking from top view) $0^{\circ}$ degree is due East, $90^{\circ}$ is North; positive angle is CCW from East towards North, negative angle is CW from East towards South.

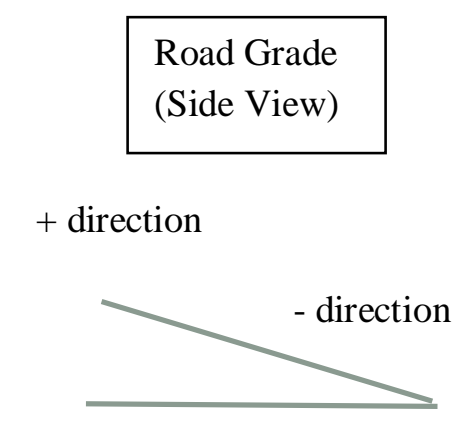

Figure 29. Vehicle Accident sign convention

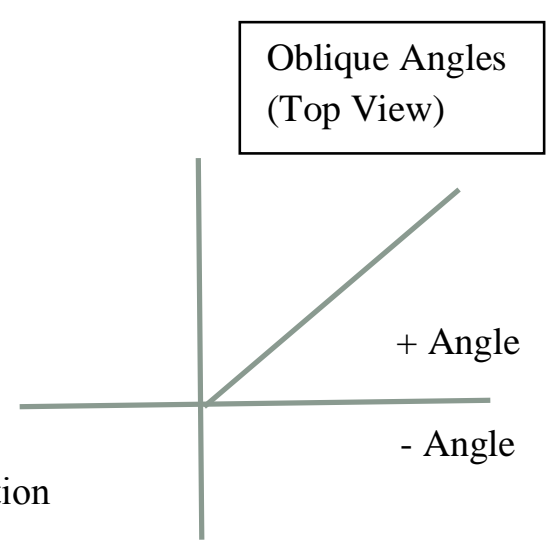

The following screenshots are the different pieces of the Excel sheet. The purple shade cells are identified as user inputs. The white shaded cells are calculated by equations in the software. Grey lettering signifies values are for checking work. 


\begin{tabular}{|c|c|c|c|}
\hline & & igeorget@calpoly.edu & bself@calpoly.edu \\
\hline & & Other Properties & \\
\hline (Purple Cells are inputs) & & units & environment \\
\hline (White Cells are calculated) & & \multirow{2}{*}{$\begin{array}{l}\text { distance meters, velocity } \mathrm{m} / \mathrm{s} \text {, } \\
\text { angle degree }\end{array}$} & road grade(deg) \\
\hline ( Enter purple parametes and then & & & 0 \\
\hline Start at bottom of page) & & & \\
\hline \multicolumn{4}{|l|}{ Car Properties } \\
\hline mass $(\mathrm{kg})$ & 1477 & mass & 8436 \\
\hline $\mathrm{cg}$ height $(\mathrm{m})$ & 0.61 & cg height & 0.61 \\
\hline bumper coeff $\left(\mathrm{N}^{*} \mathrm{~m} / \mathrm{m}\right)$ & 0 & bumper coeff & 0 \\
\hline car height (m) & 1.94 & car height (m) & 1.94 \\
\hline
\end{tabular}

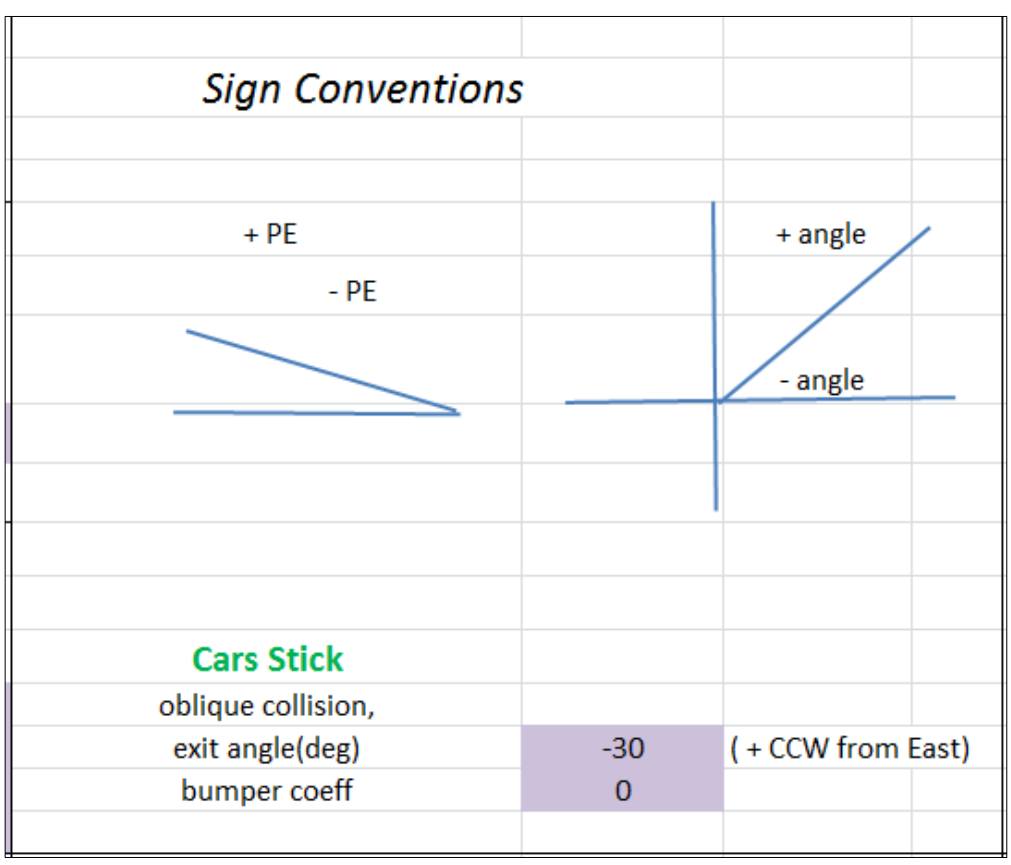




\begin{tabular}{|c|c|c|c|}
\hline \multicolumn{4}{|l|}{ Initial velocities } \\
\hline find initial veloc $(\mathrm{m} / \mathrm{s})(\mathrm{km} / \mathrm{hr})$ & 24.99 & find initial veloc $(\mathrm{m} / \mathrm{s})(\mathrm{km} / \mathrm{hr})$ & 2.153 \\
\hline $\mathrm{KE}$ & 461312.56 & KE & 19550.50 \\
\hline PE pre skid & 8829.51 & PE pre skid & 50430.41 \\
\hline Did Any Cars pre-Skid? & yes car 1 & & \\
\hline Did Car 1 Skid? & yes & Did Car 2 Skid? & no \\
\hline skid coeff & 0.67 & skid coeff & 0.5 \\
\hline skid distance (m) & 2.9 & skid distance (m) & 0 \\
\hline skid work & 28124.15 & skid work & 0.00 \\
\hline$\triangle \mathrm{PE}$ & 0.00 & $\triangle \mathrm{PE}$ & 0.00 \\
\hline KE post skid & 433188.41 & KE post skid & 19550.50 \\
\hline PE post skid,pre flip height & 8829.51 & PE post skid,pre flip height & 50430.41 \\
\hline exit velocity $(\mathrm{m} / \mathrm{s})(\mathrm{km} / \mathrm{hr})$ & 24.219 & exit velocity $(\mathrm{m} / \mathrm{s})(\mathrm{km} / \mathrm{hr})$ & 2.153 \\
\hline Did Any Cars Flip? & no car flipped & & \\
\hline Did Car 1 Flip? & no & Did Car 2 Flip? & no \\
\hline new cg height $(\mathrm{m})$ & 0.61 & new cg height & 0.61 \\
\hline exit PE & 8829.506 & exit PE & 50430.408 \\
\hline exit KE & 433188.41 & exit KE & 19550.50 \\
\hline exit Velocity $(\mathrm{m} / \mathrm{s})(\mathrm{km} / \mathrm{hr})$ & 24.22 & exit Velocity $(\mathrm{m} / \mathrm{s})(\mathrm{km} / \mathrm{hr})$ & 2.153 \\
\hline
\end{tabular}

\begin{tabular}{|c|c|c|c|}
\hline What kind of Collision? & Oblique Collision and stick & & \\
\hline Did Car 1 Collide obliquely? & yes & Did Car 2 Collide Obliquely? & yes \\
\hline enter angle (zero) & 0 & enter angle & 50 \\
\hline enter velocity & 6.000 & enter velocity & 6.409 \\
\hline pre veloc $\mathrm{x}$ & 24.219 & pre veloc $\mathrm{x}$ & 1.384 \\
\hline pre veloc y & 0.000 & pre velocy & 1.649 \\
\hline pre veloc mag & 24.219 & pre veloc mag & 2.1529 \\
\hline exit angle & -30 & exit angle & -30 \\
\hline exit velocity & 6.000 & exit velocity & 6.409 \\
\hline
\end{tabular}

The velocities found in each section are used as inputs to the next section. For example, post velocites of skid phase become pre velocities of flip phase. 


\begin{tabular}{|c|c|c|c|c|c|}
\hline $\begin{array}{c}\text { Both Cars Head on Collision and } \\
\text { stick together? }\end{array}$ & no & & $\begin{array}{l}\text { Both Cars Head on Collision and } \\
\text { go separately? }\end{array}$ & no & \\
\hline car 1 velocity pre $(\mathrm{m} / \mathrm{s})$ & 5 & user input & car 1 velocity pre $(\mathrm{m} / \mathrm{s})$ & 5.5 & user input \\
\hline car 2 velocity pre $(\mathrm{m} / \mathrm{s})$ & 4.17 & & car 2 velocity pre $(\mathrm{m} / \mathrm{s})$ & -0.96 & \\
\hline car 1 velocity post $(\mathrm{m} / \mathrm{s})$ & FALSE & & car 1 velocity post $(\mathrm{m} / \mathrm{s})$ & FALSE & \\
\hline car 2 velocity post $(\mathrm{m} / \mathrm{s})$ & FALSE & & car 2 velocity post $(\mathrm{m} / \mathrm{s})$ & FALSE & \\
\hline & & & & & \\
\hline
\end{tabular}

\begin{tabular}{|c|c|c|c|c|c|}
\hline $\begin{array}{c}\text { Did any cars post- } \\
\text { skid? }\end{array}$ & yes both cars together & 7 & & & \\
\hline pre-velocity $(\mathrm{m} / \mathrm{s})$ & 0.000 & pre-velocity (m/s) & 0.000 & pre velocity, both cars stuck $(\mathrm{m} / \mathrm{s})$ & 2.807 \\
\hline KE pre & 0.0 & KE pre & 0.0 & KE Pre & 39053.3 \\
\hline PE pre & 0.00 & PE pre & 0.00 & PE pre & 0.00 \\
\hline Did Car 1 Skid separately? & no & separately? & no & Did Cars 1-2 Skid toget & no \\
\hline skid distance $(\mathrm{m})$ & 0 & skid distance $(\mathrm{m})$ & 0 & mass total & 9913 \\
\hline$\mu \mathrm{k}$ & 0.6 & $\mu \mathrm{k}$ & 0.5 & skid distance $(\mathrm{m})$ & 0.6 \\
\hline skid work (N) & 0.00 & skid work (N) & 0.00 & $\mu \mathrm{k}$ & 0.67 \\
\hline PE change & 0.00 & PE change & 0.00 & skid work (N) & -39053.25 \\
\hline KE post & 0.00 & KE post & 0.00 & PE change & 0.00 \\
\hline PE post & 0.00 & PE post & 0.00 & KE post & 0.00 \\
\hline \multirow[t]{2}{*}{ exit velocity $(\mathrm{m} / \mathrm{s})$} & 0.000 & exit velocity $(\mathrm{m} / \mathrm{s})$ & 0.000 & PE post & 0.00 \\
\hline & & & & exit velocity $(\mathrm{m} / \mathrm{s})$ & 0.000 \\
\hline
\end{tabular}

\begin{tabular}{|c|c|c|c|c||}
\hline Did any bumper crush? & no bumper crush & & & \\
\hline Did bumper crush? & no & Did bumper crush? & no & Did bumper crush? \\
\hline crush distance $(\mathrm{m})$ & 0 & crush distance $(\mathrm{m})$ & 0 & no \\
\hline bumper work $(\mathrm{N})$ & 0.000 & bumper work $(\mathrm{N})$ & 0.000 & crush distance $(\mathrm{m})$ \\
\hline height PE change & 0.00 & height PE change & 0.00 & bumper work $(\mathrm{N})$ \\
\hline height exit PE & 0.00 & height exit PE & 0.00 & 0.000 \\
\hline exit KE (zero) & 0 & exit KE (zero) & 0 & 0.00 \\
\hline exit velocity (zero $\mathrm{m} / \mathrm{s})$ & 0 & exit velocity (zero $\mathrm{m} / \mathrm{s})$ & 0 & height $\mathrm{PE}$ change \\
\hline
\end{tabular}

